In memory of my Teacher and senior friend

Vyacheslav Borisovich Priezzhev

\title{
Untangling of Trajectories and Integrable Systems of Interacting Particles: Exact Results and Universal Laws
}

\author{
A. M. Povolotskya,, , * \\ ${ }^{a}$ Bogoliubov Laboratory of Theoretical Physics, Joined Institute for Nuclear Research, Dubna, 141980 Russia \\ ${ }^{b}$ National Research University Higher School of Economics, Moscow, 101000 Russia \\ *e-mail: alexander.povolotsky@gmail.com \\ Received August 21, 2020; revised September 9, 2020; accepted September 9, 2020
}

\begin{abstract}
This review gives a survey of some results about systems of interacting particles and the laws characterizing their behavior on large scales, which are common for a number of phenomena unified under the notion of the Kardar-Parisi-Zhang universality class.
\end{abstract}

DOI: $10.1134 / \mathrm{S} 1063779621020040$

\section{CONTENTS}

1. Introduction

2. Acknowledgments

243

3. Bethe Ansatz as untangling of trajectories 243

3.1. Vicious walkers and enumeration of non-intersecting paths

3.2. Generating functions, free fermions and imaging

3.3. Interacting particles and Bethe ansatz 248

3.3.1. Limiting cases

4. Green's functions and generating functions in the model with interaction

4.1. Determinant formulas and generalized Green function

5. Determinantal process and correlation functions

6. Asymptotic analysis: limiting

hydrodynamics and universal fluctuations

\section{INTRODUCTION}

Equilibrium statistical physics allows one to describe systems with a large number of degrees of freedom in a state of thermodynamic equilibrium. At the heart of this theory, the foundations of which were laid in the pioneering works of L. Boltzmann and J.W. Gibbs, there is an idea that a typical macroscopic system in equilibrium behaves like random, and each of its microscopic states is realized with the probability determined by the Boltzmann-Gibbs distribution, depending only on the energy and perhaps on a small set of other parameters. The entire subsequent history of the statistical physics has demonstrated an extraordinary generality of this picture.

The simplest "toy" system, which can be used to illustrate this formalism, is a lattice gas in which particles are located at the sites of the lattice $\mathscr{L}$, which is a subset of integer $d$-dimensional lattice, $\mathscr{L} \subset \mathbb{Z}^{d} \subset \mathbb{R}^{d}$, in turn embedded into the $d$-dimensional space. We assume, that each site $i \in \mathscr{L}$ can in principle contain any number of particles $n_{i} \in Z_{\geq 0}$. Then, the probability assigned to a system configuration $\mathbf{n}=\left\{n_{i}\right\}_{i \in \mathscr{L}}$ is

$$
\mathbb{P}(\mathbf{n})=\frac{1}{Z(\beta, \mu)} e^{-\beta(H(\mathbf{n})-\mu\|\mathbf{n}\|)}
$$

where $Z(\beta, \mu)$ is a partition function that plays the role of a normalization factor, $\beta$ and $\mu$ are external parameters, the inverse temperature and the chemical potential, respectively, $\|\mathbf{n}\|=\sum_{i \in \mathscr{L}} n_{i}$ is the total number of particles, and $H(\mathbf{n})$ is the energy of the particle configuration $\mathbf{n}$. Usually $H(\mathbf{n})$ is the sum of terms corresponding to interparticle interactions inside each site, between the neighboring sites, next-to-neighboring sites, etc.

The probabilistic construction leads to very general conclusions about large scale statistics of the system under consideration. Statements known in the probability theory as the law of large numbers (LLN) and the central limit theorem (CLT) can be applied to the description of some characteristics under very general assumptions about the energy of microstates [1-3]. In 
particular this concerns with the case of short range forces, i.e. when the energy of interaction between different parts of the system decreases rapidly as the distance between them grows. Then typical ${ }^{1}$ is the situation when the correlation length is finite. Roughly speaking, if we divide the system into boxes of a size greater than some characteristic size, called the correlation length, then, up to neglectable corrections, random variables $\xi_{1}, \xi_{2}, \ldots$, characterizing different boxes (for example, energy, number of particles, spin etc.) can be considered independent. Suppose also that all $\xi_{i}$ are identically distributed (this is the case if the function $H(\mathbf{n})$ is translation invariant), and their first two moments are finite,

$$
\rho=\mathbb{E} \xi_{i}, \quad \sigma^{2}=\mathbb{D} \xi_{i} .
$$

Then, for a special class of so-called additive functions of microstates, defined as the sum of a large number of independent random variables, characterizing different boxes,

$$
S_{n}=\sum_{i=1}^{n} \xi_{i},
$$

LLN and CLT will hold. Together they state that as $n \rightarrow \infty$ the asymptotics of the random variable $S_{n}$ in two leading orders consists of the linear in $n$ deterministic part and normally distributed random variable of $\operatorname{order} \sqrt{n}$,

$$
S_{n} \sim n \rho+\sqrt{n} \sigma \mathcal{N}+o(\sqrt{n}) .
$$

It is not difficult to understand the meaning of the numerical coefficients arising in this expression. The coefficient of the linear part gives the thermodynamic value of the density of the corresponding physical observable. The random part characterises the distribution of typical fluctuations of this observable near its thermodynamic value. It is important that the form of the formula (1) is universal, i.e. valid for a very wide class of quantities $\xi_{i}$, entering only through a pair of numerical parameters $\rho$ and $\sigma$. For example, if we choose $\xi_{i}$ being the number of particles in the box $i$, then $\rho$ and $\sigma$ will be expressed in terms of the derivatives of the specific free energy

$$
\mathfrak{f}(\beta, \mu)=-\lim _{\mathscr{L} \rightarrow \mathbb{Z}^{d}} \frac{\beta^{-1}}{\|\mathscr{L}\|} \ln Z(\beta, \mu)
$$

using standard thermodynamic relations

$$
\rho=\partial \tilde{f}(\beta, \mu) / \partial \mu, \quad \sigma^{2}=\beta^{-1} \partial^{2} \tilde{f}(\beta, \mu) / \partial \mu^{2} .
$$

\footnotetext{
${ }^{1}$ By typical we mean the choice of parameters away from the points of phase transitions.
}

Returning to our simple lattice gas example, consider a system in which the energy contains only the zero range interactions by setting

$$
H(\mathbf{n})=\sum_{x \in \mathscr{L}} e\left(n_{i}\right),
$$

where we assume that the function $e(n)$ decreases as $n$ increases. Then the Boltzmann-Gibbs distribution has a factorized form

$$
\mathbb{P}(\mathbf{n})=\prod_{x \in \mathscr{L}} \frac{\tilde{\mathfrak{f}}\left(n_{x}\right) e^{\beta \mu n_{x}}}{z(z)}
$$

where $\tilde{f}(n)=e^{-\beta e(n)}, z(z)=\sum_{k \geq 0} \mathrm{f}(k) z^{k}$. Now we can choose individual sites as the independent boxes with the quantities $\xi_{i}$ being the numbers of particles in them. Then the random variable that has the meaning of the total number of particles in a certain large region containing $n \rightarrow \infty$ sites will asymptotically be given by formula (1), where the parameters are expressed by formulas (2) through free energy

$$
\mathfrak{f}(\beta, \mu)=-\beta^{-1} \ln z\left(e^{\beta \mu}\right) .
$$

Note that in this extremely simplified situation the spatial position of boxes does not play any role due to complete independence of their occupation numbers. It is not difficult to add the dependence on spatial coordinates to this picture by introducing a slowly changing chemical potential $\mu=\mu(x / a)$, where the large parameter $a$ specifying the characteristic size of spatial inhomogeneity can be chosen of order of the linear system size. We now define the macroscopic density of particles $s_{a}(r)=a^{-d} S_{n(a r)} / v$ dividing the number of particles $S_{n(a r)}$ in a box of volume $a^{d}{ }_{V}$ containing point with coordinate $a r$ by the volume. In the limit $a \rightarrow \infty, V \rightarrow 0$, so that $a^{d} V \rightarrow \infty$, the density $s_{a}(r)$ asymptotically behaves as

$$
s_{a}(x) \sim \rho(x)+a^{-d / 2} \sigma(x) \eta(x)+\ldots,
$$

where $\eta(x)$ is the white noise, i.e. random Gaussian field

$$
\mathbb{E} \eta(x)=0, \quad \mathbb{E} \eta(x) \eta\left(x^{\prime}\right)=\delta^{d}\left(x-x^{\prime}\right),
$$

and the local parameters $\rho(x)$ and $\sigma(x)$ are still expressed using formulas (2) in terms of the local value of the potential $\mu(x)$ at the macroscopic scale.

We have given the simplest example of the universal behavior specific for the so-called Gaussian universality class. Being trivial in the case of zero range interaction, it has a much wider area of applicability, for example to systems with interparticle interactions of finite range or rapidly decreasing as the distance between sites or the number of particles at the sites grows. Qualitatively, this behavior is expected. in the domain of analyticity of the free energy in its variables, where the correlation length is finite. It is the finite- 
ness of the correlation length, providing the asymptotic independence of the distant parts of the system, which is responsible for the fact that the system looks like a random Gaussian field in the large scale.

In this review, we are not going to make precise statements about the limiting behavior of equilibrium systems, nor to discuss the degree of generality of the Gaussian universality class. We have used this example to demonstrate how a universal limiting picture appears in the large scale in the framework of the equilibrium statistical mechanics. It is natural to wonder what other types of universal behavior can arise when considering the multicomponent systems under coarsening. For example, in equilibrium, more complex types of universal behavior arise at the critical point in the situation of the second order phase transition, when the correlation length is infinite. Note that in this case, simple arguments like the ones above are not enough, and to obtain exact limiting distributions, it is necessary to develop sophisticated mathematical techniques allowing a description of exact statistics of models with a special integrable structure.

We will consider another important issue - universality in nonequilibrium systems. The search for examples of such universality serves as a motivation for obtaining results presented in this review. Unfortunately, there is no general approach to nonequilibrium systems similar to the Boltzmann-Gibbs theory. A common point for large multicomponent systems is that at large times they often behave as random. We accept this assumption from the very beginning, considering the simplest stochastic evolution of the model system defined by a Markov chain. Moreover, we will also consider lattice models of interacting particles, such as the lattice gas, with local Markov dynamics. The idea is to find dynamical rules that would make possible an exact calculation of rich enough spectrum of physical observables. Moving after that to the large scale consideration, i.e. taking a special scaling limit of the exact results obtained, we hope to obtain a universal scaling description applicable not only to specific exactly solvable models, but also to a whole universality class that goes beyond the narrow range of exactly solvable models.

In the end of our research, we will find the universal behavior specific for the Kardar-Parisi-Zhang (KPZ) universality class [4], which unifies many nonequilibrium phenomena as the random surface growth of various origins: boundaries between the phases of matter, crystal surfaces, boundaries of wetting, drying and combustion and etc., as well as various nonequilibrium systems of traffic flows [5]. We will describe one example of a probability distribution (TracyWidom distribution [6]) and a random process (Airy process [7]), which are a manifestation of universality in the KPZ class. Note that the universal distributions obtained appear not only in the context of non-equilibrium statistical physics. They were first discovered in the studies of the statistics of eigenvalues of random matrices [8], and in the study of the asymptotic behavior of random permutations and Young diagrams [9] describing the representation theory of large groups [10]. The same universal distributions appear in the description of universal fluctuations around the limiting shapes in equilibrium systems such as dimer packing and tilings of various flat regions [11, 12], as well as in the study of one-dimensional random walks or Brownian motion in bounded geometry [13]. Another class of equilibrium problems with similar universal scaling behavior is the problem of a polymer in a random environment. At zero temperature this is the last passage percolation problem related to the already mentioned statistics of random permutations and Young diagrams [14]. At finite temperature, the free energy distribution of a Brownian polymer in the medium, where randomness is medelled by the white noise, can be found using the replica method [15], which allows establishing a connection with an exactly solvable quantum-mechanical problem of bosons with delta interaction [16]. The solution leads to results with the same scaling behavior [17-19]. Interestingly, the free energy of the polymer in this equilibrium problem is a solution to the nonlinear stochastic KPZ equation, which essentially describes the nonequilibrium growth of an interface subject to a random force. An alternative solution to the latter, exploiting the connection with a nonequilibrium system of interacting particles, leads to the same result [20].

Our program requires several steps. The point is that an exact solution of an evolutionary problem for a system with a large number of degrees freedom is usually an insurmountable task. The exception are the models belonging to the class of so-called integrable systems. Until recently, the integrable systems considered were mainly the quantum many particle systems in space-time dimension $1+1$ or two-dimensional models of statistical physics [21]. It turns out, however, that the same structures can be found in the stochastic systems of interacting particles [22]. Indeed, if one requires a special stochasticity properties from the Hamiltonian of a quantum system, such as a Heisenberg spin chain, the evolution (Schrödinger) equation, written in imaginary time, will describe the Markov dynamics of a system of interacting particles. To draw an analogy with two-dimensional statistical physics systems, we note that often they can be formulated in terms of probabilistic (Gibbsian) measures on the set of directed lattice paths, in turn, defined in terms of the weights of local configurations. However, a twodimensional lattice can be thought of as a discretization of the $1+1$ dimensional space-time. Then, under the condition of stochasticity of the corresponding operator (transfer matrix) the paths can be considered as trajectories of interacting particles, the probability measures on which have the meaning of the transition probabilities of a Markov chain. Below we will follow this approach. 
Our first step is to construct a system of interacting particles with possibly more general dynamical rules ensuring the integrability of corresponding evolution operator. Technically, this means that the evolution operator is diagonalizable using a toolbox of methods, which are usually combined under the name Bethe ansatz (BA) [23]. Using BA, one can find a general solution to the dynamical equation describing stochastic evolution of the system. However, as a rule, it is difficult to extract information about the physics from general multipoint distributions containing complete information about a multicomponent system. Therefore the next step is the calculation of informative collective characteristics such as correlation functions or marginal distributions. This task requires application of new methods going back to the theory random matrices [8]. Finally, the last step is the asymptotic analysis of the scaling limit of the obtained exact formulas. The result is an analogue of LLN and CLT for one-dimensional random manyparticle systems.

Studies of these issues were carried out in Dubna in the group of Vyacheslav Borisovich Priezzhev over the past twenty years. We will discuss several results obtained on the way. We will begin our presentation with an explanation of which features of the Markov particle dynamics are responsible for the exact solvability of the corresponding evolution equations, wherein we will try to focus on the approach developed by Vyacheslav Borisovich, based on the so-called untangling of trajectories [24]. The main idea of this approach is that any exactly solvable dynamics of interacting particles can in a sense be reduced to the dynamics of non-interacting particles. Namely, we construct generating functions of the trajectories of interacting particles on the lattice connecting fixed sets of vertices out of the non-interacting paths, adding to paths without mutual intersections the intersecting paths with rearranged and shifted heads or ends and modified weights. First we illustrate this approach using the simplest vicious walkers model, where the elimination of configurations with intersecting paths leads to free-fermionic wave functions and Karlin-McGregor-Lindström-Gessel-Viennot [25-27] determinants. Then we move on to models with richer interactions and explore question: "For what interactions between particles the evolution of interacting particles can still be reduced to the evolution of non-interacting ones?" As a result, we arrive at a general three-parameter model of particles with a factorized stationary state containing many interesting previously known and new models as limiting cases. Construction of this model and a survey of its limiting cases is the main result, described in Section 2.

Section 3 is devoted to the construction of generating functions of paths that leads us to coordinate BA, from which, in turn, we can extract the Green functions, i.e. the probabilities of transitions between particle configurations. Similar approach that treats the
Bethe ansatz as generating functions (partition functions) of paths on a lattice was also developed in the works [28, 29]. The derivation of the Green function is reduced to calculating a Laurent series coefficient in BA. We set it out as simple as possible, neglecting mathematical rigor to focus on its combinatorial nature. Next, we turn to a simpler one-parameter case, the backward sequential update model. Green function is a determinant in this case, which we show can also be generalized to transitions between particle configurations with not only spatial but also temporal coordinates being different. Here, the concept of a boundary is introduced as a line dividing the spacetime plane into two parts, and the generalized Green function is interpreted as the probability for the particles to exit the boundary at given points.

Whereas the generalized Green function gives the total probability of the configuration of $N$ space-time positions through which $N$ particles exit the boundary, marginal distributions of positions of some of these particles are of interest. Section 4 is devoted to calculating such marginal distributions. To this end we show that the generalized Green function obtained in the previous section can be represented as the marginal distribution of a determinantal point process on an extended space. As a result, we obtain multipoint distributions of exit positions in the form of the Fredholm determinants with an explicit kernel for the system with an infinite number of particles that started to walk from a single site filled with an infinite number of particles. On the way, we also provide a simple proof of the central statement of the theory of determinantal processes Eynard-Meta theorem [30, 31], which is based on counting the non-intersecting paths with the help of Lindström-McGregor-Lindström-GesselViennot theorem.

In the last Section 5, we discuss the asymptotic analysis of the obtained exact formulas. Our goal there is not to describe the technique of asymptotic analysis, which requires the use of many technical tools, rather to give a motivation for the form of the scaling limit basing on the general principles of the KPZ theory. In particular, we describe the method of obtaining parameters included in statements similar to LLN and CLT for distributions of various random variables characterizing the particle systems and the growing interfaces associated with them, and explain the connection of these parameters with characteristics of the stationary state of infinite systems. As a result, we give the most general formulation for the scaling limit of the exit probabilities for a sequence of boundaries approximated by smooth curves from a given oneparameter family. We will complete our tour from untangling of trajectories to the universal scaling limits by formulating a result on the convergence of exit probabilities on space-like paths to a universal limiting random Airy process, thus making the most general CLT type statement for our model system, which is 
expected to be applicable to the whole KPZ universality class.

Before moving on to details, let us note that in the review we did not pursue the goal of achieving the greatest generality or rigor of presentation, just as we did not try to make an elementary introduction to the subject. Today there are many excellent reviews that do this job brilliantly [32-36]. Our modest goal is to demonstrate a somewhat more general view of some of the results, the choice of which is dictated both by the author's taste and the history of his collaboration with Vyacheslav Borisovich Priezzhev. For technical details, see [37-42].

\section{ACKNOWLEDGMENTS}

The author would like to point out the profound influence of Vyacheslav Borisovich on the development of statistical physics as well as on the personal growth of the author. The author is grateful to the providence for an opportunity to be close to a wonderful scientist, person and senior friend, to enrich spiritually from communication and interaction with him. The author is also grateful to Oleg Zaboronsky for careful reading text and fruitful discussions. The work was supported by RFBR under grant no. 19-01-00726.

\section{BETHE ANSATZ AS UNTANGLING OF TRAJECTORIES}

In this section, we describe how to construct an integrable stochastic model of interacting particles on a one-dimensional lattice. By integrability we will understand a generalization of the fact that the Markov evolution operator can be diagonalized with BA. BA was proposed by Hans Bethe [23] as an ansatz for the form of eigenfunctions of the quantum Hamiltonian of the Heisenberg spin chain. Later it turned out that the applicability of this hypothesis has deep mathematical foundations, and the analogy with the wellknown concept of Liouville integrability in classical mechanics led to the notion of quantum integrability. Development of techniques related with BA gave a powerful impetus to such an area of mathematics and mathematical physics as representation theory and led to the emergence of new areas, such as the theory of quantum groups. The BA method itself received a set different interpretations as the coordinate BA, algebraic BA, functional BA, etc. Here we would like to focus on the combinatorial approach to the coordinate BA, developed by V.B. Priezzhev. The essence of this approach is that when considering the evolution of a system of interacting particles, their trajectories can be thought of as specially weighted trajectories of noninteracting particles, while the problem of non-interacting particles decomposes into single-particle problems and, therefore, significantly simplifies.

\subsection{Vicious Walkers and Enumeration of Non-Intersecting Paths}

To illustrate this idea, we turn to the simplest model of annihilating particles on a one-dimensional lattice, known as "Vicious walkers" (VW).

Consider $N$ particles on an infinite one-dimensional lattice $\mathscr{L}=\mathbb{Z}$ in discrete time. Further, when considering this model and other ones, we will think of particles as indistinguishible and be interested only in their coordinates, which can be either strictly or weakly ordered. The corresponding sets of configurations of $N$ particles will be denoted

$$
D_{N}^{>}=\left\{\mathbf{x} \in \mathbb{Z}^{N}:\left(x_{1}>x_{2}>\cdots>x_{N}\right)\right\}
$$

and

$$
D_{N}^{\geq}=\left\{\mathbf{x} \in \mathbb{Z}^{N}:\left(x_{1} \geq x_{2} \geq \cdots \geq x_{N}\right)\right\} .
$$

In the VW model, the process starts with a certain configuration of particles with coordinates $\mathbf{x}^{0} \in D_{N}^{>}$. At every time step, each particle either makes a step to the right with probability $p$, or stands on its place with probability $(1-p)$. If two particles hit the same site, they annihilate. We will be interested only in those realizations of the random process in, which no anni-

hilation events occur at any time $\mathbf{x}(t) \in D_{N}^{>}$. Let us try to calculate the Green function for the VW model,

$$
G_{t}^{\mathrm{VW}}\left(\mathbf{x} ; \mathbf{x}^{0}\right)=\mathbb{P}^{\mathrm{VW}}\left(\mathbf{x}(t)=\mathbf{x} \mid \mathbf{x}(0)=\mathbf{x}^{0}\right),
$$

which is the probability for all $N$ particles to survive for $t$ steps and to arrive at coordinates $\mathbf{x} \in D_{N}^{>}$at time $t$. This probability can be thought of as the sum of weights of directed paths going from top to bottom and from left to right through the space-time lattice, shown in Fig. 1. The lattice has vertical and diagonal edges. The path traversing the former (the latter) edge corresponds to the fact that the particle is standing (jumping) and contributes to the weight $(1-p)$ or $p$ respectively. Different paths are independent, i.e. the weight of a group of paths is obtained as the product of the weights of individual paths. Since all particles have survived, it follows that paths could not intersect. Thus, the Green function is given by the expression

$$
G_{t}^{\mathrm{VW}}\left(\mathbf{x} ; \mathbf{x}^{0}\right)=\sum_{\substack{\{\Pi\}^{\mathrm{NI}} \\ \mathbf{x}^{0} \rightarrow \mathbf{x}}}(1-p)^{v(\Pi)} p^{d(\Pi)},
$$

where the sum is over the set $\{\Pi\}_{\mathbf{x}^{0} \rightarrow \mathbf{x}}^{\mathrm{NI}}$ of groups of $N$ directed paths connecting the sites $\left(\left(x_{1}^{0}, 0\right), \ldots,\left(x_{N}^{0}, 0\right)\right)$ and $\left(\left(x_{1}, t\right), \ldots,\left(x_{N}, t\right)\right)$ without intersections (NI-nonintersecting), and $v(\Pi)$ and $d(\Pi)$ are the numbers of vertical and diagonal edges traversed by the paths, respectively. Let us try to calculate the probability 


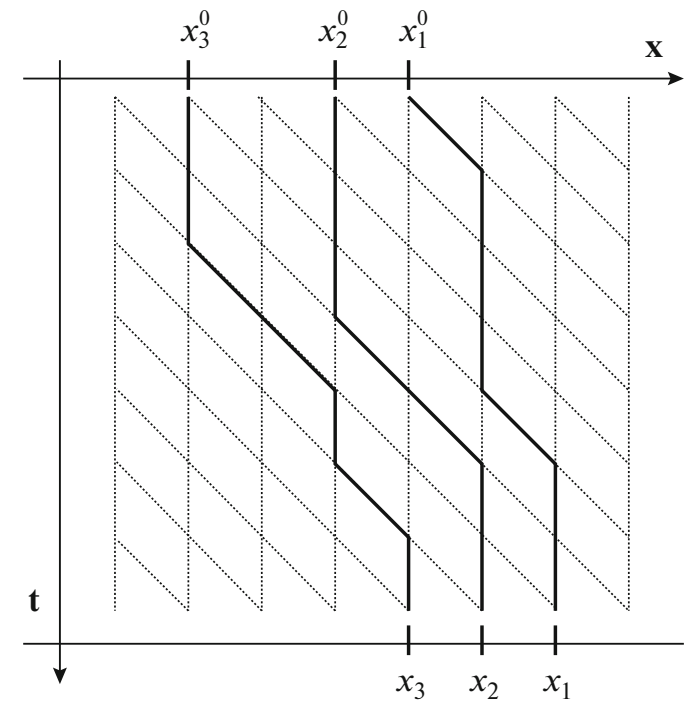

Fig. 1. Particle paths on a space-time grid. Vertical stepthe particle is standing. Diagonal step-the particle jumps.

$G_{t}\left(\mathbf{x} ; \mathbf{x}^{0}\right)$. Obviously, for one particle, $N=1$, it is given by the binomial distribution,

$$
G_{t}\left(x-x^{0}\right)=p^{x-x^{0}}(1-p)^{t-x+x^{0}}\left(\begin{array}{c}
t \\
x^{0}
\end{array}\right),
$$

i.e. the number of ways to make $\left(x-x^{0}\right)$ jumps out of $t$ steps, taken with the corresponding weight, which obviously depends only on the difference of initial and final coordinates. It is also easy to find the probability of $N$ completely independent paths pairwise connecting the sites $\left(\left(x_{1}^{0}, 0\right), \ldots,\left(x_{N}^{0}, 0\right)\right)$ and $\left(\left(x_{1}, t\right), \ldots,\left(x_{N}, t\right)\right)$, respectively. If no restrictions on such paths are assumed, then the corresponding probability will be equal to the product of one-particle probabilities,

$$
G_{t}^{\mathrm{IP}}\left(\mathbf{x} ; \mathbf{x}^{0}\right)=\prod_{i=1}^{N} G_{t}\left(x_{i}-x_{i}^{0}\right),
$$

where the superscript IP indicates that the Green function is calculated for independent particles.

To solve the problem of annihilating particles or non-intersecting paths, one needs to subtract from this product all the realizations of the process with intersections, a difficult task at first glance. However, there is a simple solution based on the KarlinMcGregor-Lindström-Gessel-Viennot theorem. (KMLGV) [25-27]. To formulate it, we introduce some definitions. Let $\mathscr{G}=(V, E)$ be a directed acyclic a graph, where each directed edge $e \in E$ is equipped with a weight $w(e)$. The weight $W(\Pi)$ of the directed path $\Pi$ passing through edges of the graph is a product of the weights of the edges,

$$
W(\Pi)=\prod_{e \in \Pi} w(e),
$$

and the weight of the transition from vertex $a$ to vertex $b$ is the sum of the weights of all paths going from $a$ to $b$ along the directed edges.

$$
w(a \rightarrow b)=\sum_{\{\Pi\}_{a \rightarrow b}} W(\Pi) .
$$

Let us generalize these definitions to groups of paths. Let $\mathbf{a}=\left(a_{1}, \ldots, a_{N}\right) \subset V$ and $\mathbf{b}=\left(b_{1}, \ldots, b_{N}\right) \subset V$ be two groups of $N$ vertices in each, which we will call heads and ends, respectively. A group of $N$ paths going from $a_{i}$ to $b_{i}, i=1, \ldots, n$, will be called $N$-path, and we will denote $\Pi_{\mathbf{a} \rightarrow \mathbf{b}}=\left\{\Pi_{a_{1} \rightarrow b_{1}}, \ldots, \Pi_{a_{N} \rightarrow b_{N}}\right)$, and the weight of an $N$-path is defined as the product of the weights of paths constituting it

$$
W\left(\Pi_{\mathbf{a} \rightarrow \mathbf{b}}\right)=\prod_{i=1}^{N} W\left(\Pi_{a_{i} \rightarrow b_{i}}\right) .
$$

Accordingly we define the transition weight $w(\mathbf{a} \rightarrow \mathbf{b})$ as the sum of the weights of all possible $N$-paths from a to b. Since paths with different heads and ends are completely independent, it's obvious that

$$
w(\mathbf{a} \rightarrow \mathbf{b})=\prod_{i=1}^{N} w\left(a_{i} \rightarrow b_{i}\right) .
$$

We also introduce the weight $w^{\mathrm{NI}}(\mathbf{a} \rightarrow \mathbf{b})$ of transition without intersections as the sum of weights of such $N$-paths from a to $\mathbf{b}$, where no two paths have vertices in common. It is clear that the last weight cannot be represented as a product, since the nonintersection condition makes the paths dependent.

\section{Theorem 1. (KMLGV)}

The following identity holds

$$
\begin{aligned}
\operatorname{det}\left\{w \left(a_{i}\right.\right. & \left.\left.\rightarrow b_{j}\right)\right\}_{1 \leq i, j \leq N}=\sum_{\sigma \in S_{N}}(-1)^{\sigma} w(a \rightarrow \sigma b), \\
& =\sum_{\sigma \in S_{N}}(-1)^{\sigma} w^{N I}(a \rightarrow \sigma b),
\end{aligned}
$$

where the sum is over all permutations of the ends, i.e. $w(\mathbf{a} \rightarrow \sigma \mathbf{b})$ is the weight of transition from $a_{1}$ to $b_{\sigma_{1}}$, from $a_{2}$ to $b_{\sigma_{2}}$ etc., and $w^{\mathrm{NI}}(\mathbf{a} \rightarrow \sigma \mathbf{b})$ means the same thing, but without intersections.

The proof of this theorem is elementary. It is based on an observation that if two paths in an $N$-path intersect at least once, then there is another $N$-path, different from the former in the parts of the two paths after the first intersection being interchanged. Obviously, the weight of these two $N$-paths coincide, but the corresponding terms enter the sum in the lhs of (5) with different signs. Thus, all such $N$-paths are canceled within the sum over permutations and the sum from the r.h.s. of (5) remains. 
To solve the problem posed about the Green function, we need the corollary from the KMLGV theorem, which, in particular, is applicable to the case of the planar lattice under consideration.

Corollary 2. If possible transitions without intersections keep the order of the paths, i.e. the only nonzero term in the lhs of (5) corresponds to the identity permutation, then

$$
w^{\mathrm{NI}}(\mathbf{a} \rightarrow \mathbf{b})=\operatorname{det}\left[w\left(a_{i} \rightarrow b_{j}\right)\right]_{1 \leq i, j \leq N} .
$$

Obviously, this is true in the case considered above, whence we have

$$
G_{t}^{\mathrm{VW}}\left(\mathbf{x}, \mathbf{x}^{0}\right)=\operatorname{det}\left[G_{t}\left(x_{i}-x_{j}^{0}\right)\right]_{1 \leq i, j \leq N} .
$$

This result can be immediately generalized to a wider classes of $N$-paths, in which not only spatial, but also temporal coordinates of heads and of ends may be different. In the corresponding processes on the one-dimensional lattice, the particles begin and end to walk at different sites and at different times. Indeed, to satisfy the conditions preserving the order of paths on a space-time lattice, it is enough that no heads and ends of the paths to be in the regions (parallelograms) visited by other paths. To formalize this statement, consider two sets of space-time points $\left(\mathbf{x}^{0}, \mathbf{t}^{0}\right)=$ $\left(\left(x_{1}^{0}, t_{1}^{0}\right), \ldots,\left(x_{N}^{0}, t_{N}^{0}\right)\right)$ and $(\mathbf{x}, \mathbf{t})=\left(\left(x_{1}, t_{1}\right), \ldots,\left(x_{N}, t_{N}\right)\right)$, such that $x_{1}>\ldots>x_{N}, x_{1}^{0}>\ldots>x_{N}^{0}, t_{i} \geq t_{i}^{0}$ for any $i=1, \ldots, N$ and there is no pair of numbers $1 \leq i \neq j \leq N$, such that the inequalities

$$
t_{i}^{0}-x_{i}^{0} \leq t_{j}^{0}-x_{j}^{0} \leq t_{i}-x_{i}, \quad x_{i}^{0} \leq x_{j} \leq x_{i},
$$

or

$$
t_{i}^{0}-x_{i}^{0} \leq t_{j}-x_{j} \leq t_{i}-x_{i}, \quad x_{i}^{0} \leq x_{j} \leq x_{i} .
$$

hold. Then the generalization of Corollary 2 is as follows

Proposition 3.

$$
\begin{gathered}
G^{\mathrm{VW}}\left((\mathbf{x}, \mathbf{t}),\left(\mathbf{x}^{0}, \mathbf{t}^{0}\right)\right)=w^{N I}\left(\left(\mathbf{x}^{0}, \mathbf{t}^{0}\right) \rightarrow(\mathbf{x}, \mathbf{t})\right) \\
=\operatorname{det}\left[G_{t_{i}-t_{j}^{0}}\left(x_{i}-x_{j}^{0}\right)\right]_{1 \leq i, j \leq N} .
\end{gathered}
$$

\subsection{Generating Functions, Free Fermions and Imaging}

Thus, we have expressed the partition function of non-intersecting paths via the antisymmetrised combination of partition functions of independent paths with permuted ends, or, equivalently, described the statistics of coordinates of interacting particles in terms of statistics of non-interacting particles. In what follows, we want to use a similar approach for paths (particles) with more complex interactions.

A handy tool for working with independent particles (paths) is the generating function of coordinates of particles (ends of paths). From point of view of the Markov chain behind particle dynamics, the generating function is a special case of the expectation of a function of configurations of a Markov chain. Let $c(t)$ is a homogeneous Markov chain ${ }^{2}$ on some finite or countable set of configurations $C$, with transition probabilities

$$
M_{c^{\prime} c}=\mathbb{P}\left(c(t+1)=c \mid c(t)=c^{\prime}\right),
$$

and given initial configuration $c_{0}$, i.e.

$$
\mathbb{P}\left(c(0)=c_{0}\right)=1 .
$$

The evolution of the expectation value of an arbitrary function of configurations $f(c)$ is given by the backward Chapman-Kolmogorov equation

$$
f_{t+1}(c)=M f_{t}(c)=\sum_{c^{\prime}} M_{c c^{\prime}} f_{t}\left(c^{\prime}\right),
$$

where

$$
f_{t}(c)=\mathbb{E}(f(c(t)) \mid c(0)=c) .
$$

If an initial probability distribution $P_{0}$ on configurations was given, then the expectation of a function $f(c)$ will look as

$$
E(f(c(t)))=E_{P_{0}}\left(f_{t}(c)\right),
$$

where the averaging on the right is over the initial distribution.

In our case, system configurations are sets of ordered particle coordinates. To reproduce the result obtained above, we introduce the generating function of their probability distribution.

$$
g_{t}\left(\mathbf{x}^{0} ; \mathbf{z}\right)=\mathbb{E}\left(\mathbf{z}^{-\mathbf{x}(t)} \mid \mathbf{x}(0)=\mathbf{x}^{0}\right)=\sum_{x \in \mathbb{Z}} G_{t}\left(\mathbf{x}, \mathbf{x}^{0}\right) z^{-\mathbf{x}} .
$$

Here the $N$-component multi-indices are again in bold: $\mathbf{x}^{0}, \mathbf{x} \in D_{N}^{>}$are the sets of ordered coordinates of heads and ends, respectively, $\mathbf{z}=\left(z_{1}, \ldots, z_{N}\right)$ is a set of parameters of the generating function, and $\mathbf{z}^{-\mathbf{x}}=z_{1}^{-x_{1}} \ldots z_{N}^{-x_{N}}$. In general, $g_{t}(\mathbf{x} ; \mathbf{z})$ is a formal Laurent power series in formal variables $z_{1}, \ldots, z_{N}$. However, in the future it will also be convenient for us to think that $z_{1}, \ldots, z_{N}$ take values in the complex plane, and the sum of the series represents a function analytic in the domain of its convergence. Then the series coefficient $\left[\mathbf{z}^{-\mathbf{x}}\right] g_{t}\left(\mathbf{x}^{0} ; \mathbf{z}\right)$ of the monomial $\mathbf{z}^{-\mathbf{x}}$,

\footnotetext{
${ }^{2}$ Our example of the model of vicious walkers, or rather its restriction on processes with a constant number of particles is strictly speaking not a Markov chain, since some of the processes drop out from consideration in course of time, violating the probability conservation. Within this section, however, the the probability conservation is not important.
} 
which is also the Green function, will be given by the contour integral

$$
\begin{gathered}
G_{t}\left(\mathbf{x}, \mathbf{x}^{0}\right)=\left[\mathbf{z}^{-\mathbf{x}}\right] g_{t}\left(\mathbf{x}^{0} ; \mathbf{z}\right) \\
=\frac{1}{(2 \pi i)^{N}} \oint_{\Gamma} \ldots \oint_{\Gamma} g_{t}\left(\mathbf{x}^{0} ; \mathbf{z}\right) \mathbf{z}^{x} \frac{d \mathbf{z}}{\mathbf{z}},
\end{gathered}
$$

where $d \mathbf{z} / \mathbf{z}=\prod_{i=1}^{N} d z_{i} / z_{i}$. If $g_{t}(\mathbf{x} ; \mathbf{z})$ is a Laurent polynomial, then the integration contour $\Gamma$ can be closed around $z_{i}=0$. In a more general case of infinite series, the integrand may contain other singular points, which in our case will not depend on the initial conditions $\mathbf{x}^{0}$. We need to fix the location of the contour with respect to singularities so that the expansion coefficients of the function $g_{t}\left(\mathbf{x}^{0} ; \mathbf{z}\right)$ in the corresponding domain match the coefficients of the formal series for the generating functions. To do this, it is enough to select a contour, such that the integral on the r.h.s. of (9) satisfies the initial condition at the time $t=0$

$$
G_{0}(\mathbf{x}, \mathbf{y})=\delta_{\mathbf{x}, \mathbf{y}}
$$

for any $\mathbf{x}, \mathbf{y} \in D_{N}^{>}$. Since for any finite time the function $g_{t}(\mathbf{x} ; \mathbf{z})$ is obtained as a finite linear combination of similar functions at the preceding moments, the choice of the integration contour does not change with time.

Next, we will try to present examples of the Markov dynamics of interacting particles for which the Green function can be calculated explicitly. We will start with the already considered example of vicious walkers, in which particle trajectories are statistically independent provided that they do not intersect, and then move on to systems with more complex interactions.

First, we write down the generating function of the distribution of coordinate $x$ for one waking particle. Action of the operator $M$ on the r.h.s. of the Chapman-Kolmogorov equation (7) to a function of coordinate in this case reads

$$
M \Psi(x)=(1-p) f(x)+p f(x+1) .
$$

For such an evolution, the generating function starting from the initial condition

$$
g_{0}\left(x^{0} ; z\right)=z^{-x_{0}}
$$

is the eigenfunction, i.e. its one time step evolution is given by multiplication

$$
g_{t}\left(x^{0} ; z\right)=\Lambda(z) g_{t-1}\left(x^{0} ; z\right)=\Lambda^{t}(z) z^{-x^{0}}
$$

by the eigenvalue

$$
\Lambda(z)=(1-p)+p / z
$$

which has a meaning of the generating function of one time step of a path.

A similar generating function for $n$ variables $\mathbf{z}=\left(z_{1}, \ldots, z_{n}\right)$, describing the evolution of $N$ indepen- dent particles, is given by the product of single-particle functions

$$
g_{t}^{\mathrm{IP}}\left(\mathbf{x}^{0} ; \mathbf{z}\right)=\prod_{i=1}^{N} g_{t}\left(x_{i}^{0} ; z_{i}\right),
$$

and, being the eigenfunction for the $N$-particle Markov matrix, evolves by multiplication by the product of one-particle eigenvalues

$$
\begin{gathered}
g_{t+1}^{\mathrm{IP}}\left(\mathbf{x}^{0} ; \mathbf{z}\right)=\Lambda_{N}(\mathbf{z}) g_{t}^{\mathrm{IP}}\left(\mathbf{x}^{0} ; \mathbf{z}\right)=\Lambda_{N}^{t}(\mathbf{z}) \mathbf{z}^{-\mathbf{x}^{0}}, \\
\Lambda_{N}(\mathbf{z})=\prod_{i=1}^{N} \Lambda\left(z_{i}\right) .
\end{gathered}
$$

Following the recipe of the previous subsection, we define the antisymmetric (AS) combination of such functions,

$$
g_{t}^{\mathrm{AS}}\left(\mathbf{x}^{0} ; \mathbf{z}\right)=\sum_{\sigma \in S_{n}}(-1)^{\sigma} g_{t}^{\mathrm{IP}}\left(\sigma \mathbf{x}^{0} ; \mathbf{z}\right)
$$

where the antisymmetrization is carried out over the permutations of the initial positions ${ }^{3}$. Coefficient of $\mathbf{z}^{-\mathbf{x}}$ in $g_{t}^{\mathrm{AS}}\left(\mathbf{x}^{0} ; \mathbf{z}\right)$ is nothing but the partition function of paths antisymmetrized with respect to the position of their initial vertices, and is exactly equal to the expression (6) for $G_{t}^{\mathrm{Vw}}\left(\mathbf{x}, \mathbf{x}^{0}\right)$ obtained above using the KMLGV theorem. Note that the generating function in the sense of the definition (8) is only a part of the antisymmetrized function $g_{t}^{\mathrm{AS}}\left(\mathbf{x}^{0} ; \mathbf{z}\right)$, in which the exponents of the monomials $\mathbf{z}^{-\mathbf{x}}$ belong to $D_{N}^{>}$, i.e. ordered as in (3) with respect to the bases. In what follows, we will call such monomials physical, and the rest non-physical. Although the non-physical terms are meaningless, adding them, we have ensured that the evolution of the resulting function $g_{t}^{\mathrm{AS}}\left(\mathbf{x}^{0} ; \mathbf{z}\right)$ coincides with that of the free Green function (11). This is due to the fact that the function $g_{t}^{\mathrm{AS}}\left(\mathbf{x}^{0} ; \mathbf{z}\right)$ is a linear combination of free functions $g_{t}^{\mathrm{IP}}\left(\mathbf{x}^{0} ; \mathbf{z}\right)$ with rearranged heads (or, what is the same, rearranged parameters $\left.z_{1}, \ldots, z_{n}\right)$, and the eigenvalue $\Lambda_{n}(\mathbf{z})$ of the Markov matrix is a symmetric function of the parameters.

In the above construction, we were guided by the idea of global re-weighting independent paths, leading to the elimination of their unwanted realizations. The same result can be achieved by considering evolution of independent particles locally at one step of Markov dynamics. Then we want to adhere to the same ideology as before. We look for a function evolving as free in the form of a linear combination of free Green functions with permuted initial vertices and require the part of this function to consist of physical monomials

\footnotetext{
${ }^{3}$ Above, we used the summation over permutations of the ends of the paths that, however, play exactly the same role as the heads.
} 
$\mathbf{z}^{-\mathbf{x}}$ that give the Green function for a system of interacting particles.

Consider the action of the Markov operator $M$ on an arbitrary function of configuration $f(\mathbf{x})$. In the vicious walkers model the particle dynamics coincides with the free one, when particles are not in neighboring sites. For example, for two particles with coordinates $x_{1}>x_{2}+1$ we will have

$$
\begin{gathered}
M f\left(x_{1}, x_{2}\right)=p^{2} f\left(x_{1}+1, x_{2}+1\right)+p(1-p) \\
\times\left(f\left(x_{1}+1, x_{2}\right)+f\left(x_{1}, x_{2}+1\right)\right)+(1-p)^{2} f\left(x_{1}, x_{2}\right) .
\end{gathered}
$$

The form of the r.h.s. changes in the case $x_{1}-1=x_{2}=x$, when the particles are in the neighboring sites

$$
\begin{gathered}
M f(x+1, x)=p^{2} f(x+2, x+1) \\
+p(1-p) f(x+2, x,)+(1-p)^{2} f(x+1, x) .
\end{gathered}
$$

One can see that the two expressions formally differ by the presence of the term $\Psi(x+1, x+1)$, whose argument is outside the range $x_{1}>x_{2}$. Therefore, to define the dynamics of vicious walkers in the whole range of particle coordinates we can restrict ourselves to the free Eq. (13), providing it with the boundary condition

$$
f(x+1, x+1)=0 .
$$

Let us set the initial conditions in the form of a linear combination of the initial conditions for free generating functions with permuted initial vertices,

$$
g_{0}\left(\left(x_{1}^{0}, x_{2}^{0}\right) ;\left(z_{1}, z_{2}\right)\right)=z_{1}^{-x_{1}^{0}} z_{2}^{-x_{2}^{0}}+A z_{1}^{-x_{2}^{0}} z_{2}^{-x_{1}^{0}},
$$

so that it is consistent with $g_{0}^{\mathrm{VW}}\left(\left(x_{1}, x_{2}\right) ;\left(z_{1}, z_{2}\right)\right)=$ $z_{1}^{-x_{1}} z_{2}^{-x_{2}}$ on physical monomials. From the boundary condition (15) it follows that $A=-1$, and we again come to the antisymmetric function $g_{t}^{\mathrm{AS}}\left(\left(x_{1}^{0}, x_{2}^{0}\right) ;\left(z_{1}, z_{2}\right)\right)$, announced above.

For $n$ particles, the situation is exactly the same. The action of the evolution operator of noninteracting particles can be written in the form

$$
\begin{gathered}
M f\left(x_{1}, \ldots, x_{n}\right)=\sum_{k_{1}, \ldots, k_{n} \in\{0,1\}} p^{\|k\|}(1-p)^{n-\|k\|} \\
\times f\left(x_{1}+k_{1}, \ldots, x_{n}+k_{n}\right),
\end{gathered}
$$

where $\|k\|=k_{1}+\ldots+k_{n}$, while the boundary conditions will have exactly the same form as in the case of two particles

$$
f(\ldots, x, x, \ldots)=0 .
$$

Let us set the initial condition in the form

$$
g_{0}(\mathbf{x} ; \mathbf{z})=\Psi^{\mathrm{BA}}(\mathbf{x} ; \mathbf{z}),
$$

where the function $\Psi_{n}^{\mathrm{BA}}(\mathbf{x} ; \mathbf{z})$ is defined by

$$
\Psi_{n}^{\mathrm{BA}}(\mathbf{x} ; \mathbf{z})=\sum_{\sigma \in S_{n}} A_{\sigma}(\sigma \mathbf{z})^{-\mathbf{x}},
$$

and the abbreviation BA in the superscript is an indication of its future use as the Bethe ansatz. This initial condition agrees with $g_{0}^{\mathrm{VW}}(\mathbf{x} ; \mathbf{z})=\mathbf{z}^{-\mathbf{x}}$ on the physical monomials corresponding to the identity permutation, when $A_{I d}=1$, and the remaining $A_{\sigma}$ do not depend on $\mathbf{z}$. The boundary condition (17) indeed implies the form of the coefficients $A_{\sigma}$,

$$
A_{\sigma}=(-1)^{\sigma}
$$

which leads to the initial condition for the antisymmetrized function (12),

$$
g_{0}(\mathbf{x} ; \mathbf{z})=g_{0}^{\mathrm{AS}}(\mathbf{x} ; \mathbf{z}),
$$

each term of which evolves as the generating function of paths of free particles by multiplying by $\Lambda_{n}^{t}(\mathbf{z})$

$$
g_{t}^{\mathrm{AS}}(\mathbf{x} ; \mathbf{z})=\Lambda_{n}^{t}(\mathbf{z}) g_{0}^{\mathrm{AS}}(\mathbf{x} ; \mathbf{z}) .
$$

Note also that due to the simple form (19) of the amplitudes $A_{\sigma}$ the initial condition can be written as a determinant

$$
g_{0}^{\mathrm{AS}}(\mathbf{x} ; \mathbf{z})=\operatorname{det}\left\{z_{i}^{-x_{j}}\right\}_{1 \leq i, j \leq N},
$$

in which we recognize Slater's determinant, i.e. the wave function of free fermions on the lattice. Occurrence of the Slater's determinant in enumeration of the nonitersecting paths was first discovered by Fisher [43]. Coefficient of the physical monomial $\mathbf{z}^{-\mathbf{x}}$ can be calculated as an $n$-dimensional contour integral (9), reducible to the determinant (6), which we have already obtained, using the KMLGV theorem.

Note that a key fact for generalizing the exactly solvable dynamics of two particles to the systems with an arbitrary number of particles is that all multiparticle interactions are reduced to the two-particle boundary conditions. As we have seen, the latter completely fixed the freedom arising from the introduction of arbitrary coefficients $A_{\sigma}$, whereas the presence of any additional conditions would make the problem overdetermined. Exactly the same requirements will further ensure the applicability of BA to systems with more complex interactions.

Another way to think about the developed formalism is to draw a parallel with the method of images originating from electrostatics and having many applications to the theory differential equations. The essence of this method applied to our problem will become clear if we reformulate the one-dimensional motion of $N$ particles with coordinates from $D_{N}^{>}$or $D_{N}^{\geq}$as the problem of the walks of one particle in $N$-dimensional positive orthant (or Weyl chamber), 
i.e. domain of $N$-dimensional lattice, in which the coordinates $\left(x_{1}, \ldots, x_{N}\right)$ of the particle are ordered in the same way. Far from the boundaries of the orthant, the particle performs the free random walk-either stands or jumps, so that any of its coordinates can increase by one. What happens when it hits the border of the othant depends on the imposed boundary conditions. In the particular case of the VW model, the particle gets absorbed upon reaching the border. This situation can be modeled by considering particle walks in the whole space, divided into $N$ ! orthants, obtained from the original orthant by reflections with respect to the boundaries. For the resulting walk in the positive orthant to be corresponding to the walks with absorbing boundaries, we will also send walking particles starting from the images of the original position in each of the orthants, with the weight having a sign determined by the ordering of coordinates in this orthant. Summing up all the weights of walks in the positive orthant leads to a description of the walks with absorbing boundaries. Next, we will try to find the most general class of models of interacting particles corresponding to more general boundary conditions. It turns out that this can be achieved not only by adding mirror images of the original source, but also by adding new sources. This generalization turns out to be equivalent to BA.

\subsection{Interacting Particles and Bethe Ansatz}

Consider particle walks on an infinite lattice. Now we will consider weakly ordered particle coordinates

$$
\mathbf{x}(t) \in D_{N}^{2},
$$

i.e. several particles may occupy the same site. On one hand, this will allow us to restrict ourselves to the simplest type of interactions, zero range interactions. On the other hand, as will be explained below, using simple transformations, from such systems one can obtain systems with finite-range and even with long-range interactions. When particles are far away from each other, they move to the right as free, according to the rules defined above. The dynamics changes, when several particles come into one site. The following dynamical rules will be understood as zero range interaction. If at some step, $k$ particles are at one site, then at the next step $0 \leq m \leq k$ particles can jump to the next site on the right with probability $\varphi(m \mid k)$ depending only on the number of particles in this site. Here $0 \leq \varphi(m \mid k) \leq 1$ is a set of probabilities, satisfying the normalization condition $\sum_{0 \leq m \leq k} \varphi(m \mid k)=1$. We will assume the parallel update, i.e. at every step decisions about how many particles will jump from each site are made at all sites simultaniously and independently. Since the rules for jumping from unoccupied sites and sites with a single particle coincide with the definition of dynamics for noninteracting particles, we have

$$
\varphi(0 \mid 0)=1, \quad \varphi(0 \mid 1)=(1-p), \quad \varphi(1 \mid 1)=p .
$$

We have to define such a family of probabilities $\varphi(m \mid k)$, which will allow us to write out many-particle equations for the generating functions as free equations equipped with a system of consistent boundary conditions. Consistency in this case means that all multiparticle interactions are specified through twoparticle boundary conditions. Just like it was in the VW model, two-particle boundary conditions fix all the freedom that appears due to the summation of generating functions over permutations of initial coordinates. Therefore, multiparticle interactions should not lead to any new restrictions.

We will implement this program step by step. Oneparticle problem is free, i.e. is the same as discussed earlier (10). Next, we consider the two-particle case, and then turn to the system with an arbitrary number of particles. We write the action of the Markov matrix $M$ on the function of configurations of two particles with coordinates $x_{1} \geq x_{2}$. When $x_{1}>x_{2}$, i.e. particles are in different sites, the action of the matrix $M$ describes the independent free evolution of two particles and, as in (13), it is equivalent to independent action of single-particle matrices for each coordinate separately. Interaction comes into play when both particles hit the same site, $x_{1}=x_{2}=x$. Then we will have

$$
\begin{gathered}
M f(x, x)=\varphi(0 \mid 2) f(x, x) \\
+\varphi(1 \mid 2) f(x+1, x)+\varphi(2 \mid 2) f(x+1, x+1) .
\end{gathered}
$$

This identity will be equivalent to the identity (13) for two non-interacting particles if the function $f\left(x_{1}, x_{2}\right)$ satisfies boundary condition

$$
f(x, x+1)=\alpha f(x+1, x+1)+\beta f(x+1, x)+\gamma f(x, x),
$$

where

$$
\begin{gathered}
\alpha=\frac{\varphi(2 \mid 2)-p^{2}}{p(1-p)}, \beta=\frac{\varphi(1 \mid 2)-p(1-p)}{p(1-p)}, \\
\gamma=\frac{\varphi(0 \mid 2)-(1-p)^{2}}{p(1-p)} .
\end{gathered}
$$

These boundary conditions must be understood as a way to express the term $f(x, x+1)$, arising from the formal writing of the free equation and lying outside the domain of the particle coordinates $D_{2}^{\geq}$.

The next step is to write equations for an arbitrary number of particles. The free equation, applicable when all particles are in different sites, $x_{i} \neq x_{j}$ for any $1 \leq i \neq j \leq N$, coincides with (16). To account for the interaction, one has to write down the action of the Markov matrix for the case, when several particles are in one site. Since all sites are updated independently, it suffices to consider updating a single site. Let $k$ par- 
ticles be found at the site with coordinate $x$. Action of the Markov matrix on the corresponding function of configuration will look as follows

$$
M f\left(\ldots, x^{k}, \ldots\right)=\sum_{m=0}^{k} \varphi(m \mid k) f\left(\ldots,(x+1)^{m}, x^{k-m}, \ldots\right),(
$$

where $x^{k}=(x, \ldots, x)$ denotes a string of $k$ particle coordinates, located in the site $x$. Note that if we formally write down the free Eq. (16) for this case, it will contain unwanted terms outside the coordinate domain (20). We would like the free equation to be reduced to the form (21) by successive application of two-particle boundary conditions.

$$
\begin{gathered}
f(\ldots, x, x+1, \ldots)=\alpha f(\ldots, x+1, x+1, \ldots) \\
+\beta f(\ldots, x+1, x, \ldots)+\gamma f(\ldots, x, x, \ldots) .
\end{gathered}
$$

It is clear that for the given probabilities $\varphi(m \mid k)$ with $k=1,2$ this requirement unambiguously fixes all the other probabilities with $k>2$. Taking into account the normalization, one can see that there are only three free parameters in the problem, which can be chosen as $p$ and any two of $\alpha, \beta, \gamma$, while the third parameter is determined from the condition

$$
\alpha+\beta+\gamma=1 \text {. }
$$

The problem of finding the probabilities $\varphi(m \mid k)$ can be given a purely algebraic form. To this end, let us look at the sequences of coordinates $(x+1)$ and $x$ in the function arguments on the right-hand sides of formulas (16) and (21). Associate them with the products of non-commuting elements $A$ and $B$, respectively, and write their sums with coefficients of the corresponding functions. Then from the formula (16) we obtain the sum of all possible products of $k$ elements with coefficients $p^{m}(1-p)^{k-m}$, where $m$ is the number of operators $A$ in the product. The formula (21) yields the sum of monomials of the form $A^{m} B^{k-m}$, in which all the letters $A$ are to the left of $B$, with coefficients $\varphi(m \mid k)$. The equality between the right-hand sides of (21) and (16) looks like the non-commutative analogue of Newton's binomial

$$
(p A+(1-p) B)^{k}=\sum_{m=0}^{k} \varphi(m \mid k) A^{m} B^{k-m},
$$

while the analog of the boundary condition (22) will have the form

$$
B A=\alpha A A+\beta A B+\gamma B B .
$$

Then the problem of finding probabilities $\varphi(m \mid k)$ is reformulated as the problem of finding the binomial coefficients of the generalized binomial formulas (24), where $A$ and $B$ are two generators of the associative algebra satisfying the homogeneous quadratic relation (25) with parameters $\alpha, \beta, \gamma$, two of which are arbitrary, and the third is found from the relation (23).
The last question was first answered in [44] and independently rediscovered by the author in the context of integrable models of interacting particles in [42]. Expressed via new parameters $q, \nu$ and $\mu$, which give convenient parameterization for $\alpha, \beta, \gamma$ and $p$,

$$
\begin{gathered}
\alpha=\frac{v(1-q)}{1-q v}, \quad \beta=\frac{q-v}{1-q v}, \\
\gamma=\frac{1-q}{1-q \nu}, \quad \mu=p+v(1-p),
\end{gathered}
$$

the result is represented as

$$
\varphi(m \mid n)=\mu^{m} \frac{(v / \mu ; q)_{m}(\mu ; q)_{n m}}{(v ; q)_{n}} \frac{(q ; q)_{n}}{(q ; q)_{m}(q ; q)_{n m}},
$$

where $(a ; q)_{k}=(1-a)(1-q a) \ldots\left(1-q^{k-1} a\right)$ is $q$-Pochhammer's symbol. We also assume that the values of $\alpha, \beta, \gamma$ are chosen such that $\nu \neq q^{-k}$, for any $k \in \mathbb{N}$. Under this condition, the algebraic statement is valid for any complex values of the parameters $\mu, v, q$. For the numbers $\varphi(m \mid k)$ to have a meaning of probabilities, they must be in the range $0 \leq \varphi(m \mid k) \leq 1$. For this, it is enough that $\mu, v$ and $q$ were real and satisfy the inequalities $|q|<1$ and $v<\mu<1$. Below we will consider this particular range of parameters.

The resulting three-parameter family turns out to be quite general, and contains many both previously studied and new models as limiting cases. Before discussing the dynamics of the models and their physical applications, we will discuss the properties of the process constructed.

To begin with, we note that the resulting hopping probabilities have the form

$$
\varphi(m \mid k)=\frac{v(m) w(k-m)}{\mathrm{f}(k)},
$$

where

$$
v(k)=\mu^{k} \frac{(v / \mu ; q)_{k}}{(q ; q)_{k}}, \quad w(k)=\frac{(\mu ; q)_{k}}{(q ; q)_{k}},
$$

and

$$
\mathrm{f}(k)=\sum_{n=0}^{k} v(n) w(k-n)
$$

provides the normalization of the probabilities $\varphi(m \mid k)$. In our case this sum can be calculated explicitly as the ratio of two $q$-Pochhammer symbols.

$$
\mathrm{f}(k)=\frac{(v ; q)_{k}}{(q, q)_{k}} .
$$

As shown in the work [45], the factorized form (28) of hopping probabilities ensures factorization of the stationary measure in the model at infinite or finite periodic lattice. 


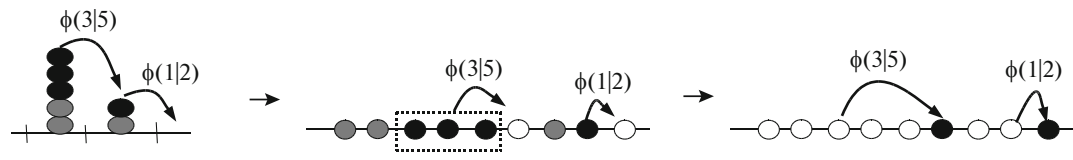

Fig. 2. ZRP-EP and particle-hole dualities. The first one maps a site containing $n$ particles to a group of $n$ sites, one particle in each, plus one empty site ahead. The second duality swaps the site with particles and holes.

Namely, the model, in which $N$ particles live on a periodic lattice with $L$ sites, evolves to a stationary state, where the probability of a configuration with $n_{1}, \ldots n_{L}$ particles at sites numbered $1, \ldots, L$, respectively, is factored into the product of single-site weights

$$
\mathbb{P}_{s t}\left(n_{1}, \ldots, n_{L}\right)=\frac{1}{Z(N, L)} \prod_{i=1}^{L} \mathrm{f}\left(n_{i}\right),
$$

where the weight $\mathrm{f}(n)$ coincides with the normalization of the hopping probabilities (30), and the partition function $Z(N, L)$ is the general normalization factor obtained by summing the products of single-site weights over all configurations with the condition $n_{1}+\ldots+n_{L}=N$. The latter condition leads to weak correlations between different sites, which vanish in the limit $L \rightarrow \infty$ in the case of sufficiently good onesite weights. As a result, the translation invariant stationary measures of our model on the infinite lattice are determined by one-site distributions of the form

$$
\mathbb{P}_{s t}\left(n_{i}=k\right)=\frac{z^{k} \mathrm{f}(k)}{z(z)},
$$

where $z$ is the activity parameterizing the stationary measure, $\mathrm{f}(k)$ is the one-site weight from (30) and $z(z)=\sum_{k=z}^{\infty} z^{k} \mathrm{f}(k)$ is the one-site partition function. The activity parameter $z$ is related to the mean particle density $\rho=\mathbb{E} n_{i}$ through the first equation from (2), where activity is related to chemical potential by the relation $z=e^{\beta \mu}$. In this case, the occupation numbers in different sites are independent. One can see that the stationary state of our model has the same form as the Gibbs measure in the example considered in the introduction. However, as it will become clear later, the nonequilibrium dynamics reveals a completely new specificity. Note that the search for a general exactly solvable dynamics leading to a factorized stationary state served as a motivation for the formulation of the model in the work [42]. In this review, we approached the problem from another side, addressing exact calculation of the generating function using BA, while the factorized form of the stationary measure came out as a by-product. However, we mention this fact here, since it will be useful for the analysis of the behavior of the system on a large scale later on.

3.3.1. Limiting cases. Consider the relationship of the constructed three-parameter family with other known models of interacting particles. To this end, we first formulate two dualities that establish a connection between particle systems, which at first glance may seem very different. The first one, ZRP-EP duality, connects systems like zero range process (ZRP), where the number of particles at a site is unlimited, with exclusion processes (EP), in which the interaction between neighboring sites prohibits coming more than one particles into one site. The transformation consists in replacing a site occupied with $n$ particles by a compact cluster of $n$ sites, one particle in each, plus one more empty site ahead (see Fig. 2). Accordingly, $m$ particles leaving a site with $n$ particles for the next site in a ZRP-like system corresponds to the shift of a cluster of $m$ particles, detached from a cluster, of $n$ particles in a EP-like system. The number of sites in a finite EP-like system is equal to the sum of the number of sites and the number of particles in the ZRP-like system.

The second correspondence, the particle-hole transformation, connects the two EP-like systems, swapping occupied and free sites. A particle jumping $m$ steps in one of the systems corresponds to a shift of a cluster of $m$ particles by one step in the opposite direction in the other.

We also point at the fact that from the discrete time models one can often go to continuous time models sending jumping probability to zero. This can be done in different ways and with different limiting results. What is common, however, is that the set of models becomes narrower, i.e. from various models in discrete time we often arrive at the same continuous time model. The easiest way to take the continuous time limit in our model is to consider simultaneous limits $\mu \rightarrow v$, i.e. $p \rightarrow 0$, and $t \rightarrow \infty$, such that the new continuous time $\tau=t p$ remains finite. If the rest of the parameters are kept constant, instead of the discrete time jumps we obtain Poissonian jumps of $m$ particles from a site with $n$ particles with the rate

$$
u(m \mid n)=\frac{\mu^{m-1}}{1-q^{m}} \frac{(\mu, q)_{n-m}(q, q)_{n}}{(\mu, q)_{n}(q, q)_{n-m}} .
$$

Processes with similar hopping rates called q-Hahn $\mathrm{ZRP}^{4}$ were studied in the work [46], where, however, their generalization with jumps in both directions were considered. In [48] it was shown that the corresponding Markov matrix can be interpreted as the Hamilto-

\footnotetext{
${ }^{4}$ The prefix q-Hahn was first used when applied to a model with probabilities jumps (27) in the work [47].
} 
nian of the XXZ chain, the model of Heisenberg magnet with non-compact spin symmetry group.

Other limits, both discrete and continuous, can also be obtained by imposing restrictions on the parameters of probabilities $\varphi(m \mid n)$. Here is a list of models obtained from our hopping probabilities (27) as a result of various limiting transitions.

Free particles, $q=1$. For $q=1$, the dependence on $\mu$ and $\nu$ separately disappears, and there remains the binomial distribution with the parameter $p$

$$
\lim _{q \rightarrow 1} \varphi(m \mid n)=\varphi_{\text {Bin }}(m, n ; p)=p^{m}(1-p)^{n m}\left(\begin{array}{l}
n \\
m
\end{array}\right) .
$$

These hopping probabilities describe a system of particles making independent directed Bernoulli random walks. Every particle tries to jump one step forward with probability $p$. Binomial coefficient takes into account the number of ways to select $m$ out of $n$ particles in a site. The limit of continuous time described above leads us from the Bernoulli random discrete time random walks to Poisson random walks.

Random walks in a random environment ( $R W R E)$, $q, \mu=q^{\alpha}, v=q^{\alpha+\beta} \rightarrow 1$. In this limit, the hopping probabilities approach the beta-binomial distribution

$$
\begin{gathered}
\lim _{q=\mu^{1 / \alpha}=v^{1 /(\alpha+\beta)} \rightarrow 1} \varphi(m \mid n)=\varphi_{\mathrm{B}-\mathrm{Bin}}(m, n ; \alpha, \beta) \\
=\frac{B(\alpha+m, \beta+n m)}{B(\alpha, \beta)}\left(\begin{array}{c}
n \\
m
\end{array}\right),
\end{gathered}
$$

which is a convolution

$$
\varphi_{\mathrm{B}-\mathrm{Bin}}(m, n ; \alpha, \beta)=\int_{0}^{1} \varphi_{\mathrm{Bin}}(m, n ; p) \varphi_{\mathrm{B}}(p ; \alpha, \beta) d p
$$

of the binomial distribution $\varphi_{\text {Bin }}(m, n ; p)$ with the beta distribution

$$
\varphi_{\mathrm{B}}(p ; \alpha, \beta)=\frac{p^{\alpha-1}(1-p)^{\beta-1}}{B(\alpha, \beta)} \rrbracket_{p \in[0,1]} .
$$

The binomial distribution indicates that the jumps in this model are still performed independently. However, the convolution with the beta distribution should be interpreted as the randomness of the parameter $p$ of the Bernoulli distribution, which is chosen independently from the beta distribution at every new update of every site. In other words, we obtained the system of particles making independent RWRE, with disorder simulated by the random beta-distributed probability of the particle jump from a site. The Beta binomial hopping probabilities obtained are already the result of averaging over the disorder. This model was studied in [49], where the problem of RWRE was also interpreted in the language of the rigorous replica method applied to the problem of polymer in a random environment. Solution of the problem of the disorder-averaged probability of $n$-particle transition between two sites, corresponding to the $n$-replica polymer partition function, allows one to construct a Fredholm determinant expression for the Laplace transform of the distribution of the random transition probability for a particle in a random environment with "beta" disorder. In physical language, such a probability corresponds to the random partition function of the polymer in random enviroment for some special initial conditions. An asymptotic analysis of this result gives the limiting distribution, which describes the height of the interface evolving according to the Kardara-Parisi-Zhang equation.

TASEP with generalized update, $q=0$. For $q=0$, the particles in the ZRP model jump from the site occupied by $n$ particles with probabilities $\varphi(0 \mid n)=(1-p), \varphi(m \mid n)=(1-\mu) p \mu^{m-1}$ for $0<m<n$ and $\varphi(n \mid n)=p \mu^{n-1}$. It is convenient to give an interpretation of this process in the language of a exclusion process. After ZRP-EP transformation we obtain the totally asymmetric version of the EP (TASEP) with jumps from left to right and cluster-oriented backward sequential update, in which the particle entering the site remembers whether this site was occupied in the previous step or not. During the update, each cluster is scanned from right to left. The first particle tries to jump one step forward with probability $p$. If successful, the second one tries to jump one step forward with probability $\mu$, generally different from $p$. The same happens with the third, fourth particle, etc. If some particle in this sequence refuses to jump, all subsequent particles of the same cluster stand with the probability one. For $\mu=p(v=0)$, we obtain the usual TASEP with (cluster-oriented) backward sequential update, whereas $\mu=0$ corresponds to the model with parallel update, where all particles make a decision simultaneously basing on the current configuration. In the range $0<\mu<1$, the effective interaction varies from repulsive to attracting, and in the limit $\mu \rightarrow 1$ corresponds to the model with deterministic aggregation, in which all particles finally stick together into one cluster. In the limit $p \rightarrow 0$ under the corresponding time scaling one obtains the model of cluster fragmentation in continuous time, where a cluster of particles can decay by Poissonian jump into two clusters, when the first few particles take a step forward, and each next particle follows the previous one with probability $\mu$. Setting also $\mu=0$, we obtain the TASEP in the continuous time, where particles independently perform Poissonian jumps forward, when the site ahead is not occupied. Models with $q=0$ belong to a special class of models, in which the transition probabilities can be represented as a partial distribution of a determinant process, a probability measure on point configurations, which can be completely characterized by a hierarchy of multipoint correlation functions having simple determinant form. This relationship allows one to calculate explicitly multipoint distributions of 
positions of particles in the TASEP and conduct their asymptotic analysis. We will briefly describe these calculations in the following sections.

Asymmetric diffusion with multiparticle hopping and model with the long range jumps, $v \rightarrow \mu=q$. Here we consider the continuous time limit $p=d t \rightarrow 0$ with the special condition $\mu=q$, under which the hopping probabilities are reduced to the form $\varphi(m \mid n) \simeq d t /[m]_{1 / q}$, where the index $1 / q$ shows that the deformation parameter in the $q$-number is equal to

$q^{-1}$, rather than $q$. A model with similar jump probabilities was suggested in [50]. However, it allowed jumping in both directions with a degree of asymmetry, depending on the parameter $q$. Its generalization, in which the dependence of the probability jump on the number of particles in a site was independent of the degree of asymmetry, was considered in [51]. The totally asymmetric version of the latter is a special case of our model in the considered limit.Via ZRP-EP transformation we obtain the model proposed in [52], where a particle pushes forward a cluster of particles with the rate $r_{n}=1 /[n]_{1 / q}$, depending on the number $(n-1)$ of particles in front of it. This model gives interpolation between TASEP and "drop-push" model [53] obtained in the limits $q \rightarrow \infty$ and $q \rightarrow 0$, respectively. It was also shown in [54] that the limit $q \rightarrow 1$ of this model taken together with the continuous space limit yields the integral Hamiltonian obtained in [55], which previously appeared in problems of quantum chromodynamics.

q-boson ZRP, q-TASEP and the asymmetric avalanche process, $\mu=q v$. In this case, the first q-Pochhammer symbol $(v / \mu ; q)_{m}$ vanishes for $m>1$. Therefore, only jumps of one particle from a site are allowed. The resulting process is a discrete-time ZRP, in which one particle jumps from a site occupied by $n$ particles with probability $\varphi(1 \mid n)=p \cdot[n]_{q}$. The corresponding integrable q-boson model, was first proposed in [56] in the language of the algebraic Bethe ansatz. Its Hamiltonian (continuous time) version was discussed later in [57]. It was considered again in [58], where the following question was posed: "What are the most common jump probabilities that make the ZRP integrable?" Later, the same question was addressed to the model of discrete-time ZRP [59]. As before, the continuous time model can be obtained from the discrete time version in the limit $p \rightarrow 0$. In the model with discrete time, the parameters can take values in the range $|q|<1,0<p<1$, whereas in the case of continuous time, $q$ can be any real number. In the latter case, the limit $q \rightarrow \infty$ corresponds to the "drop-push" model, in which a particle jumps to the nearest unoccupied site after an exponentially distributed waiting time or, equivalently, pushes all the right neighbors one step forward. After successive ZRP-EP and particle-hole transformations the q-boson ZRP becomes the so-called q-TASEP, in which $\varphi(1 \mid n)$ is the probability for a parti- cle, from which the closest particle to the left is $n$ empty sites ahead, to jump one step left. This model was recently obtained as a limiting case of the Macdonald process [60] and used to study behavior of a polymer in a random environment [61].

Another continuous time model, the asymmetric avalanche process (AAP) [62], appears in the limit $v \rightarrow 0$ for $-1<q<0$. Setting $1-p=d t \rightarrow 0$ and passing into a reference frame that moves one step forward each time step, we obtain a model of the EP type, in which transitions between configurations are governed by instantaneous non-local avalanches. In the moving reference frame the dynamics is as follows. Starting from an arbitrary configuration of particles, in which no more than one particle is allowed at any site, some particle jumps a step forward after an exponentially distributed waiting time. If the next site is occupied by another particle, the latter either joins the incoming particle, in which case they together take a step forward with probability $\left(1-[2]_{q}\right)$, or do not join, and then the incoming particle jumps forward alone. This is how the avalanche starts, whereupon it develops in discrete time infinitely fast from the point of view of continuous Poissonian time. In continuous time the avalanche ends instantly and is included only into the transition rates between configurations of EP type. In the general case, if at some step of an avalanche $n>1$ particles are in a site, then either all $n$ particles go ahead with probability $(1-[n])$, or one particle remains, while the other $(n-1)$ particles go forward. Thus, at each step, the number of particles in the avalanche can either increase or decrease by one, or remain unchanged. The avalanche ends, when one particle from a pair enters an unoccupied site. The interest in this model was inspired by the transition from dispersive to continuous flow that occurs in an infinite system at the particle density $\rho_{c}=1 /(1-q)$. In the limit $q \rightarrow 0$, the model obtained from the AAP is again "drop-push".

Geometric q-TASEP, $v=0$. In [63] two generalizations of the TASEP were proposed. In one of them, called the geometric q-TASEP, a particle jumps forward to any unoccupied site, if no occupied sites are on the way to it, with the probability $p_{n, \mu}(l)$ of the jump length $l$ depending on the distance to the next occupied site, which is obtained from $\varphi(l \mid n)$ at $v=0$. This process can be obtained from ours using the ZRP-EP and particle-hole transformations. Note that this process was obtained in [63] as a reduction of the Macdonald process, and the technique used was limited to the case of step initial conditions. The question was also raised there about the possibility of applying the Bethe ansatz technique to this process, which would allow considering arbitrary initial conditions. Answer to this question was given in [58], and the hopping probability proposed there turned out to be even more general, depending on three parameters. 
We can also consider other special cases of our model, in which transition probabilities have a simpler form. The above are those that have arisen in the literature earlier. In addition, several models were suggested with partially asymmetric dynamics, such as the two-parameter long jump model [52], multiparticle diffusion with exclusion [50], "Push-ASEP" [64] and AAP with two-sided jumps [65]. They cannot be obtained as special cases of our completely asymmetric model. However, given the generality of the model, one can expect that its transfer matrix may be a generating function of various Hamiltonians, including those corresponding to jumps in both directions, see for example [66].

Also, it is necessary to mention several important developments and generalizations of our model. First, these are vertex models with higher spins [67], formulated in terms of directed paths on a square lattice, in which the number of paths passing along vertical and horizontal edges are bounded by integer parameters $I$ and $J$. The weights of the vertices in this model have a more complex form, being expressed in terms of qRacah polynomials, and its transfer matrix is obtained by the convolution of our transfer matrix with special selected parameters with similar transposed transfer matrix. Conversely, the transfer matrix of our model is restored under some special analytic continuation of the weights with respect to the parameters $I$ and $J$. We will also note the already mentioned q-Hahn TASEP with double-sided jumps [46] and q-Hahn pushTASEP [68].

\section{GREEN'S FUNCTIONS AND GENERATING FUNCTIONS IN THE MODEL WITH INTERACTION}

Although the problems similar to computing Green functions, such as computing partition functions of vertex models on a bounded domain with fixed boundary conditions, were studied before, the first result in the context of stochastic models of interacting particles was obtained by Schuetz for the continuous time TASEP [69]. It has been shown that in this case, the Green function has a determinant form. The same determinantal form is also acquired by the Green function in the case of discrete-time TASEP with backward sequential update [37], also called the fragmentation process [70]. The next step was taken in [39], where the Green function in the form of a ratio of two determinants was obtained for the first time for a system with a nontrivial stationary state, TASEP with parallel update. Subsequently this computation has been generalized for TASEP with generalized update [71]. All these examples can be obtained as special cases of the limit $q \rightarrow 0$ of the model obtained above. As will be clear below, in this case the transitions probabilities are distinguished by a special Markov property: the evolution of a particle depends only on particles with smaller numbers but not on those with larger ones. Technically, this leads to a special factorization of the scattering amplitude, because of which the formulas are greatly simplified, and the Green functions have the determinantal form.

For models that do not have this property, the problem was first solved by Tracy and Widom for EP with partially asymmetric dynamics (ASEP) [72]. For a q-boson ZRP, the Green function was first obtained in [73] as a result of solving a more general problem of constructing Plancherel's theory for the eigenfunctions of the Markov generator. A conjecture for the form of Green function for our general three-parameter model was proposed in [42], and proved in [47], where the Plancherel theory was also constructed for the corresponding eigenfunctions. Below, we first illustrate the idea of [47] for the general $q$, and then discuss the simplification in the case $q=0$.

Let us turn to the problem of constructing generating functions of paths. Let us describe the evolution of expectation

$$
g_{t}(\mathbf{x} ; \mathbf{t})=\mathbb{E}\left(g_{0}(\mathbf{x}(t) ; t) \mid \mathbf{x}(0)=\mathbf{x}\right)
$$

of functions with the initial condition taken in the form of BA,

$$
g_{0}(\mathbf{x} ; \mathbf{z})=\Psi_{N}^{\mathrm{BA}}(\mathbf{x} ; \mathbf{z}),
$$

according to the formula (18), and we require it to satisfy boundary condition (22). As a result, we get a recurrent relation for the coefficients $A_{\sigma}$

$$
S\left(z_{i}, z_{j}\right) \equiv \frac{A_{\ldots i j \ldots} \ldots}{A_{\ldots j i \ldots}}=-\frac{\alpha+\beta z_{i}+\gamma z_{i} z_{j}-z_{j}}{\alpha+\beta z_{j}+\gamma z_{i} z_{j}-z_{i}},
$$

which, given the initial condition $A_{i d}=1$ for the identity permutation, results in the general expression

$$
A_{\sigma}=\prod_{1 \leq i<j \leq N: \sigma_{j}<\sigma_{i}} S\left(z_{\sigma_{i}}, z_{\sigma_{j}}\right) .
$$

For the function $S\left(z_{1}, z_{2}\right)$ we will use the term scattering amplitude that conveys the meaning of this quantity in the context of particle scattering in quantum mechanics.

The key difference between this result and the freefermion case of VW is $A_{\sigma}$ now being a function of variables $z_{1}, \ldots, z_{N}$. To interpret $g_{t}(\mathbf{x} ; \mathbf{z})$ in the language of generating functions of paths, one needs to turn the whole function into Laurent series. This procedure will add many new paths to the already introduced paths with permuted origins. Multiplication of $(\sigma z)^{-\mathbf{x}}$ by monomials from the Laurent expansions gives new paths with the heads shifted comparing to the original ones. Among them the physical ones, i.e. those in which the exponents are ordered according to (20), will constitute the desired generating function of the interacting paths.

Note that the procedure of expanding a meromorphic function into a Laurent series is not unique. The 
result depends on the domain of the complex planes of variables $z_{1}, \ldots, z_{N}$, in which this or that expansion converges. Which monomials are contained in the initial conditions for the generating function, and, accordingly, the generating function of which quantity is obtained as a physical part of it depends on the form of expansion of expressions (34). The reasoning below should be understood as one of recipes for transforming the rational functions (33), (34) into the Laurent series. After this expansion is obtained, one can think about it as a formal series, i.e. set of expansion coefficients. Indeed, the evolution of the function $g_{t}^{\mathrm{BA}}(\mathbf{x} ; \mathbf{z})$ by any finite number of time steps is reduced to multiplying the initial functions by a polynomial. The corresponding linear transformations of the coefficients affect only a finite number of terms of the series and, therefore, is well defined. The same is true for the boundary conditions (22), which ensure that the physical part of this function evolves as a generating function that describes a system of interacting paths.

Next, we obtain the expansion of $g_{t}(\mathbf{x} ; \mathbf{z})$, in which the only physical monomial will be proportional to $\mathbf{z}^{-\mathbf{x}}$. To turn $S\left(z_{1}, z_{2}\right)$ into the Laurent series, it is convenient to use representation (26) of parameters $\alpha, \beta$ and $\gamma$ in terms of $v$ and $q$ and write the scattering amplitude in the form

$$
S\left(z_{1}, z_{2}\right)=\frac{q-\frac{\left(z_{1}-1\right)}{\left(z_{1}-v\right)} \frac{\left(z_{2}-v\right)}{\left(z_{2}-1\right)}}{1-q \frac{\left(z_{1}-1\right)}{\left(z_{1}-v\right)} \frac{\left(z_{2}-v\right)}{\left(z_{2}-1\right)}} .
$$

Let us consider the case $0<v<1$, although the general conclusions are apparently valid also for any values of $v<1$. We will assume that the variables $z_{1}$ and $z_{2}$ take values on the circle $\left|z_{i}-1\right|=\epsilon\left|z_{i}-v\right|$, where $v<\epsilon<1$. With this choice, the ratio multiplied by $q$ in the denominator is equal in modulus to one. Bearing in mind that $|q|<1$, we expand the fraction, as a geometric progression in powers $q \frac{\left(z_{1}-1\right)}{\left(z_{1}-v\right)} \frac{\left(z_{2}-v\right)}{\left(z_{2}-1\right)}$, after which we note that the contour can be deformed without passing through the poles $z=1, v$ so that it is in the ring $v<|z|<1$. In such a ring, each term of the resulting series can be expanded in nonnegative powers of $z_{1}^{-1}$ and $z_{2}$. Calculating the first sum term by term, we arrive at the following expansion

$$
\begin{gathered}
S\left(z_{1}, z_{2}\right)=\sum_{m, l \geq 0} z_{1}^{-m} z_{2}^{l} s_{l, m}=\frac{q-v}{v q-1} \\
-z_{2} \frac{\left(1-q^{2}\right)((1-v)}{(1-q v)^{2}}-\frac{1}{z_{1}} \frac{(1-v) v\left(1-q^{2}\right)}{(1-v q)^{2}} \\
+\frac{z_{2}}{z_{1}} \frac{\left(1-q^{2}\right)(1-v)^{2}(1+q v)}{(1-q v)^{3}}+\ldots
\end{gathered}
$$

The key point for the further analysis is the presence of only non-positive powers of the first argument and non-negative powers of the second one in this expansion.

To work with multiparticle generating functions, one also needs to consider the products of several such expansions. Such manipulations are not completely flawless from the point of view of the mathematical theory of formal series, since the series contain an infinite number of terms with both positive, and negative powers. In general, algebraic operations with such series are reduced to calculating infinite sums of products of their coefficients and are not always well defined. However, in our case, the result is unambiguous, the resulting infinite sums converge, and can be computed explicitly. This is a consequence of the fact that our series are received as expansions of specific functions in the domains of their analyticity. The fact also based on this is the possibility of presenting the final expression for the Green function in the form of a contour integral along the contours located in these domains. The detailed analysis of the analytical structure of the expressions can be found in [47], while in our presentation we will adhere to the principles of economy, sacrificing, where it is justified, mathematical rigor. In particular, depending on convenience we will think of the same objects either as of formal series or as about analytic functions.

Let us give an interpretation of the physical part of the function $g_{t}(\mathbf{x} ; \mathbf{z})$ and, in particular, its value $g_{0}(\mathbf{x} ; \mathbf{z})$ at the initial moment. Note that the term in $\Psi^{\mathrm{BA}}(\mathbf{x} ; \mathbf{z})$, corresponding to the permutation of the origins of the paths $\sigma$, is given by the monomial $(\sigma \mathbf{z})^{-\mathbf{x}}$, multiplied by the coefficient $A_{\sigma}$, which can be thought of as the product of functions $S\left(z_{\sigma_{i}}, z_{\sigma_{j}}\right)$ written as the expansion (35). Moreover, the product in (34) is the product over inversions of the permutation $\sigma$ : it contains only the factors corresponding to pairs of numbers $i<j$, for which inequality $\sigma_{i}>\sigma_{j}$ holds. In other words, unlike the initial order in the physical configuration, where particle numbers increase from right to left, in such pairs the particle with the higher number is in the same position or to the right of the particle with the lower number. From the form of expansion (35) one can see that the multiplication of the monomial by $S\left(z_{\sigma_{i}}, z_{\sigma_{j}}\right)$ generates new monomials, in which the particle with number $\sigma_{i}\left(\sigma_{j}\right)$ either stays in place, or moves to the right (left).

As an example, let us try to find the physical monomials of the function $\Psi_{2}^{B A}\left(\left(x_{1}, x_{2}\right) ;\left(z_{1}, z_{2}\right)\right)$ in the case of two particles on the lattice. In addition to the physical monomial, corresponding to the identity permutation, the sum (18) contains the monomial corresponding to the only nontrivial permutation $\sigma=(21)$, the transposition, swapping the particles at sites $x_{1}$ and $x_{2}$. It 
is obvious that if particles are at different sites, $x_{1}>x_{2}$, the permutation reverses their order, transforming the physical monomial $z_{1}^{-x_{1}} z_{2}^{-x_{2}}$ into a nonphysical monomial $z_{1}^{-x_{2}} z_{2}^{-x_{1}}$. Next, multiplying the latter by $S\left(z_{2}, z_{1}\right)$, we obtain that with the coefficient $\frac{q-v}{v q-1}$ plus monomials $z_{1}^{-\left(x_{2}-1\right)} z_{2}^{-x_{1}}, z_{1}^{-x_{2}} z_{2}^{-\left(x_{1}+1\right)}, z_{1}^{-\left(x_{2}-1\right)} z_{2}^{-\left(x_{1}+1\right)}$ etc. with the corresponding expansion coefficients from (35). Thus, the multiplication of the nonphysical monomial by $S\left(z_{2}, z_{1}\right)$ can only aggravate the situation by increasing (decreasing) the exponent of $z_{1}\left(z_{2}\right)$, thereby shifting the right (left) particle to the right (to the left), so that the added monomials will still be nonphysical. An exception is the case, $x_{1}=x_{2}=x$, in which the transposition does not change the monomial $\left(z_{1} z_{2}\right)^{-x}$, and from the monomials of the expansion of $S\left(z_{2}, z_{1}\right)$ only the free term does not violate the physical order of exponents. As a result, the coefficient of the only physical monomial $\mathbf{z}^{\mathbf{x}}$ in $\Psi_{2}^{\mathrm{BA}}\left(\left(x_{1}, x_{2}\right) ;\left(z_{1}, z_{2}\right)\right)$ will look as

$$
\begin{aligned}
& {\left[\mathbf{z}^{-\mathbf{x}}\right] \Psi_{2}^{\mathrm{BA}}\left(\left(x_{1}, x_{2}\right) ;\left(z_{1}, z_{2}\right)\right) } \\
= & z_{1}^{-x_{1}} z_{2}^{-x_{2}}\left(1-\delta_{x_{1}, x_{2}} \frac{q-v}{v q-1}\right) .
\end{aligned}
$$

In this example, we saw that multiplication of the monomials corresponding to permutations of particles by the scattering amplitudes shifts the particles that were moved by an inversion to the left (right) even more to the left (more to the right), respectively. For an arbitrary number of particles and permutations, corresponding to multiple inversions, the same particle can play the role of the right and left particle in different inversions simultaneously. In this case, it is multiplied by several scattering amplitudes, in which the corresponding variable can be either the first or the second argument, and the corresponding particle is sequentially shifted both to the right and to the left. Although the overall picture is rather complex, the rightmost (leftmost) of the inverted particles are still shifted in only one direction, even more to the right (left), respectively. Therefore, if there is at least one inversion in the permutation affecting particles at different sites, multiplication of the corresponding nonphysical monomial by the scattering amplitudes will generate only non-physical monomials. The only exceptions are permutations, changing the order of particles inside sites, but not rearranging particles between sites. For the same reason, among the displacements of particles from a site generated by the scattering amplitudes only those result in physical monomials, which bring the particles back to their original sites. The result can be formulated as follows. Let $\mathbf{x}$ and $\mathbf{y}$ be two physical sets of coordinates. Then

$$
\left[\mathbf{z}^{-\mathbf{y}}\right] \Psi_{N}^{\mathrm{BA}}(\mathbf{x} ; \mathbf{z})=\delta_{\mathbf{x}, \mathbf{y}} C(\mathbf{x}),
$$

i.e. the only physical monomial in $\Psi_{N}^{\mathrm{BA}}(\mathbf{x} ; \mathbf{z})$ is proportional to $\mathbf{z}^{-\mathbf{x}}$, where $C(\mathbf{x})$ is the coefficient depending on $\mathbf{x}$, which arose as a result of summation over the permutations of particles inside sites.

To calculate $C(\mathbf{x})$ we first note that $C(\mathbf{x})=1$ for the configuration $\mathbf{x}=\left(x_{1},>\ldots,>x_{N}\right)$, in which all particles are at different sites, since in this case all the summands in (18), except the one corresponding to the identity permutation contain only nonphysical monomials. For an arbitrary configuration $\mathbf{x}$ of the form

$$
\begin{gathered}
\mathbf{x}=\left(x_{1} \ldots=x_{n_{1}}>x_{n_{1}+1}=\ldots\right. \\
\left.=x_{n_{1}+\ldots+n_{k-1}}>x_{n_{1}+\ldots+n_{k-1}+1} \ldots=x_{n_{1}+\ldots+n_{k}}\right),
\end{gathered}
$$

in which there are $k$ occupied sites with $n_{1}, \ldots, n_{k}$ particles, the summation in (18) over permutations inside different sites is performed independently. Therefore, $C(\mathbf{x})$ is factorized into the product of single-site factors

$$
C(\mathbf{x})=\prod_{i=1}^{k} c\left(n_{i}\right)
$$

where $c(n)$ is the result of summation over permutations of $n$ particles inside a site. Since permutations within the sites do not actually change physical monomial, to find the coefficient, it is enough to calculate the free term of products of the corresponding scattering amplitudes

$$
c(n)=[\mathrm{CT}] \sum_{\sigma \in S_{n}} \prod_{\left\{1 \leq i<j \leq n \mid \sigma_{j}<\sigma_{i}\right\}} S\left(z_{\sigma_{i}}, z_{\sigma_{j}}\right),
$$

where $[C T] f$ is a constant term, i.e. term of degree zero in the series $f$. To calculate $c(n)$, we first rewrite the product under the sum as

$$
\prod_{\left\{1 \leq i<j \leq n \mid \sigma_{j}<\sigma_{i}\right\}} S\left(z_{\sigma_{i}}, z_{\sigma_{j}}\right)=\operatorname{sgn}(\sigma) \prod_{1 \leq i<j \leq n} \frac{u_{\sigma_{i}}-q u_{\sigma_{j}}}{u_{i}-q u_{j}},
$$

where for brevity we have introduced new variables

$$
u_{i}=\frac{z_{i}-1}{z_{i}-v} .
$$

Since the denominator is independent of the permutation, it suffices to calculate the sum in the numerator,

$$
\begin{gathered}
\sum_{\sigma \in S_{n}} \operatorname{sgn}(\sigma) \prod_{1 \leq i<j \leq n}\left(u_{\sigma_{i}}-q u_{\sigma_{j}}\right) \\
=\frac{(q ; q)_{n}}{(1-q)^{n}} \prod_{1 \leq i<j \leq n}\left(u_{i}-u_{j}\right) .
\end{gathered}
$$

To prove this identity we note that the summation result will be a homogeneous polynomial of degree $n(n-1) / 2$, antisymmetric in all $n$ variables. Up to a common factor, the only such a polynomial is $\Delta(\mathbf{u})=\prod_{1 \leq i<j \leq n}\left(u_{i}-u_{j}\right)$. The common factor can be found by calculating the coefficient of any monomial, 
for example, for $u_{1}^{n-1} u_{2}^{n-2} \ldots u_{n-1}$. Expanding brackets in each term, we find the coefficients of such monomials given by products of $n(n-1) / 2$ factors, each of which is either one, or $-q$, depending on whether the first or second term from this bracket was included in the monomial. For example, in the term corresponding to the identity permutation only the first terms are included into the monomial $u_{1}^{n-1} u_{2}^{n-2} \ldots u_{n-1}$, so that the corresponding coefficient is equal to one. For a nonidentical permutation, we represent the permutation, as a list of inversions. Each inversion replaces the first term from corresponding parenthesis to the second one and, accordingly, the unit in the product by $-q$. Thus, in the term corresponding to the permutation $\sigma$, the coefficient of a given monomial will be equal to $(-q)^{\# \operatorname{inv}(\sigma)}$, where $\# \operatorname{inv}(\sigma)$ is the number of inversions in $\sigma$. Noticing that the sign of this coefficient compensates for the sign $\operatorname{sgn}(\sigma)$ under sum over permutations, we find that the coefficient of interest is nothing but the generating function of the number of inversions, which is explicitly computed as a q-factorial [74]

$$
\sum_{\sigma \in S_{n}} q^{\# \operatorname{inv}(\sigma)}=\frac{(q ; q)_{n}}{(1-q)^{n}} .
$$

Now, in the initial variables, our task is reduced to finding the free term in the expansion of simple rational function

$$
c(n)=\frac{(q ; q)_{n}}{(1-q)^{n}}[C T] \prod_{1 \leq i<j \leq n} \frac{1-\frac{\left(z_{i}-v\right)}{\left(z_{i}-1\right)} \frac{\left(z_{j}-1\right)}{\left(z_{j}-v\right)}}{1-q \frac{\left(z_{i}-v\right)}{\left(z_{i}-1\right)} \frac{\left(z_{j}-1\right)}{\left(z_{j}-v\right)}}
$$

We will calculate it step by step. First, we calculate the part of the expansion with zero power of $z_{n}$, then its part with zero power of $z_{n-1}$ etc. According to the expansion recipe formulated above, the variable $z_{n}$ is included in the right-hand side of (37) only in powers less than or equal to zero. Therefore, the zero-degree term $z_{n}$ is given by the limit of this expression at $z_{n} \rightarrow \infty$.

$$
\begin{aligned}
c(n)= & \frac{(q ; q)_{n}}{(1-q)^{n}}[\mathrm{CT}] \prod_{k=1}^{n-1} \frac{1-v}{1-v q} \frac{1}{1-z_{k} \frac{(1-q)}{(1-v q)}} \\
& \times \prod_{1 \leq i<j \leq n-1} \frac{1-\frac{\left(z_{i}-v\right)}{\left(z_{i}-1\right.} \frac{\left(z_{j}-1\right)}{\left(z_{j}-v\right)}}{1-q \frac{\left(z_{i}-v\right)}{\left(z_{i}-1\right)} \frac{\left(z_{j}-1\right)}{\left(z_{j}-v\right)}}
\end{aligned}
$$

Now, in the second product on the right side $\mathrm{z}_{n-1}$ appears only in powers less than or equal to zero. However, since $\left|\frac{(1-q)}{(1-v q)}\right|<1$, the factors of the first product are expanded in positive powers $z_{k}$ for $\left|z_{k}\right|<1$. To find the free from $z_{n-1}$ term one needs to multiply two such series. A simple form of the first factor allows this calculation to be carried out explicitly. To this end, we use the following simple consequence of the Cauchy integral formula. Let $f(z)=\sum_{k=0}^{\infty} z^{-k} f_{k}$ is a series in non-positive powers, converging absolutely for $|z|>1 / a-\varepsilon$ for some $a, \varepsilon>0$. Then

$$
\begin{gathered}
{[\mathrm{CT}] \frac{1}{1-a z} f(z)=[\mathrm{CT}] \sum_{k=0}^{\infty} f_{k} z^{-k} \sum_{l=0}^{\infty}(a z)^{l}} \\
=\sum_{l=0}^{\infty} f_{l} a^{l}=f(1 / a) .
\end{gathered}
$$

Thus, the calculation of the $z_{n-1}$-free term is reduced to the change $z_{n-1} \rightarrow \frac{(1-v q)}{(1-q)}$ in (38) or, equivalently, $\frac{\left(z_{n-1}-1\right)}{\left(z_{n-1}-v\right)} \rightarrow q$.

$$
\begin{gathered}
c(n)=\frac{(q ; q)_{n}}{(1-q)^{n}} \frac{1-v}{1-v q}[\mathrm{CT}] \prod_{k=1}^{n-2} \frac{1-v}{1-v q^{2}} \\
\times \frac{1}{1-z_{k} \frac{\left(1-q^{2}\right)}{\left(1-v q^{2}\right)}} \prod_{1 \leq i<j \leq n-2} \frac{1-\frac{\left(z_{i}-v\right)}{\left(z_{i}-1\right)} \frac{\left(z_{j}-1\right)}{\left(z_{j}-v\right)}}{1-q \frac{\left(z_{i}-v\right)}{\left(z_{i}-1\right)} \frac{\left(z_{j}-1\right)}{\left(z_{j}-v\right)}}
\end{gathered}
$$

The last expression has a form similar to (38) up to the change $q \rightarrow q^{2}$ in the first product, which allows one to repeat the replacement $\frac{\left(z_{n-k}-1\right)}{\left(z_{n-k}-v\right)} \rightarrow q^{k}$ recursively for $k=2,3, \ldots$, which brings us to the final result

$$
c(n)=\frac{(q ; q)_{n}}{(v ; q)_{n}}\left(\frac{1-v}{1-q}\right)^{n} .
$$

It turns out that the result obtained can be expressed in terms of single-site stationary weights

$$
c(n)=\frac{\mathrm{f}(1)^{n}}{\mathrm{f}(n)}
$$

and the overall coefficient is reciprocal to the stationary weight of a particle configuration

$$
C(\mathbf{x})=\mathrm{f}_{s t}^{-1}(\mathbf{x})=\mathrm{f}(1)^{N} \prod_{i \in L} \mathrm{f}^{-1}\left(n_{i}\right)
$$

normalized so that the weight of those configurations in which all particles are in different sites, would be equal to one.

Recalling the discussion of generating functions at the beginning of this section, we conclude that our function starting from the initial condition

$$
g_{0}(\mathbf{x} ; \mathbf{z})=\Psi_{N}(\mathbf{x} ; \mathbf{z})=\mathbf{z}^{-\mathbf{x}} \mathrm{f}_{s t}^{-1}(\mathbf{x})+(\text { non-phys }),
$$


where non-physical monomials are collected in (nonphys), evolving as free, comes to expression

$$
\begin{gathered}
g_{t}(\mathbf{x} ; \mathbf{z})=\Lambda_{n}^{t}(\mathbf{z}) g_{0}(\mathbf{x} ; \mathbf{z}) \\
=\mathbb{E} g_{0}(\mathbf{x}(t) \mid \mathbf{z})=\mathbb{E} \frac{\mathbf{z}^{-\mathbf{x}(t)}}{\mathrm{f}_{s t}(\mathbf{x}(t))}+\text { (non-phys), }
\end{gathered}
$$

the physical part of which is the generating function of the ratio of the probabilities of particle configurations to their stationary weight $f_{s t}(\mathbf{x}(t))$. Accordingly, the Green function is given by the expression

$$
\begin{gathered}
G_{t}\left(\mathbf{x} ; \mathbf{x}^{0}\right)=\mathrm{f}_{s t}(\mathbf{x})\left[\mathbf{z}^{-\mathbf{x}}\right] \Lambda_{N}^{t}(\mathbf{z}) \Psi^{B A}\left(\mathbf{x}^{0} ; \mathbf{z}\right) \\
=\mathrm{f}_{s t}(\mathbf{x}) \oint \ldots \oint \Lambda_{N}^{t}(\mathbf{z}) \Psi_{N}^{\mathrm{BA}}\left(\mathbf{x}^{0} ; \mathbf{z}\right) \mathbf{z}^{\mathbf{x}} D \mathbf{z},
\end{gathered}
$$

which can be represented as a multiple contour integral along circles centered at the origin of radius $v<|z|<1$, where by the measure of integration we mean $D \mathbf{z}=\prod_{i=1}^{N} d z_{i} /\left(2 \pi i z_{i}\right)$.

\subsection{Determinant Formulas and Generalized Green Function}

When $q=0$, the formulas obtained for the Green function acquire a simple determinant form. The reason for this is a special factorized scattering amplitude form

where

$$
S\left(z_{1}, z_{2}\right)=-\frac{h\left(z_{1}\right)}{h\left(z_{2}\right)}
$$

$$
h(z)=\frac{z-1}{z-v}
$$

Due to this factorization, multiple integrals of each term in $\Psi^{\mathrm{BA}}\left(\mathbf{x}^{0} ; \mathbf{z}\right)$ in the r.h.s. of $(40)$ decouple, reducing to a product of single integrals. As a result, the Green function turns into the ratio of determinants of $N \times N$ matrices.

$$
G_{t}\left(\mathbf{x} ; \mathbf{x}_{0}\right)=\frac{\operatorname{det}\left\{F_{i-j}\left(x_{i}-x_{j}^{0}, t\right)\right\}_{0 \leq i, j \leq N}}{\operatorname{det}\left\{F_{i-j}\left(x_{i}-x_{j}, 0\right)\right\}_{0 \leq i, j \leq N}} .
$$

Their matrix elements are specified in terms of function

$$
\begin{gathered}
F_{n}(x, t)=\frac{1}{2 \pi \mathrm{i}} \oint_{\Gamma_{0}, v}(h(z))^{-n} \Lambda^{t}(z) z^{x-1} d z \\
=\frac{1}{2 \pi \mathrm{i}} \oint_{\Gamma_{0}, v} \frac{(1-u \mu)^{t}(1-u v)^{x t-1}}{u^{n}(1-u)^{x+1}} d u \\
=\frac{(1-v)(x-1)_{n}}{n !} F_{1}(-n ;-t, t-x+1 ; 2-x n ; \mu, v),
\end{gathered}
$$

defined by the contour integral of a rational function. In the first line of this formula, the integration is performed along the contour enclosing singularities $z=0, v$. After changing the variables $u=h(z)$, this integral reduces to the integral in the second line along the contour around $z=0$, which is explicitly calculated as a terminating series for the hypergeometric Appell function $F_{1}\left(-n ; \beta, \beta^{\prime} ; \gamma ; x, y\right)$ of two variables.

Until now, we were interested in the probabilities of transitions between configurations of particles at fixed times. However, thinking about the Green function as the partition function of directed paths on the lattice, one can generalize the obtained formulas for transitions in which the initial and final vertices of the paths form more complex space-time sets. Such a statement of the problem first appeared in [37] and was subsequently used in $[40,41]$ to study the correlation functions of currents and probabilities of exit of particle trajectories from the specified space-time domains in TASEP with backward sequential update $(q=v=0)$. Below we will summarize these results. Details of the calculations can be found in the works [37, 40, 41].

Let us define the set of admissible $N$-point configurations on the space-time lattice

$$
\begin{gathered}
\mathscr{D}_{N}=\left\{(\mathbf{x}, \mathbf{t}): \mathbf{x}=\left(x_{1} \geq \ldots \geq x_{N}\right),\right. \\
\left.\mathbf{t}=\left(t_{1} \leq \ldots \leq t_{N}\right)\right\} .
\end{gathered}
$$

In general, the points of such configurations can be located on a staircase-like lines, straight sections of which run along vertical and horizontal lattice edges from right to left and from top to bottom.

$$
\begin{gathered}
\mathscr{B}=\left\{b(\tau) \in \mathbb{Z}^{2}: b(\tau+1)=(x-1, t)\right. \\
\text { or } b(\tau+1)=(x, t+1)\}_{\tau \in \mathbb{Z}} .
\end{gathered}
$$

We call boundary a set of sites of a square lattice on such a line. In this case, we will say that the configuration $(\mathbf{x}, \mathbf{t})$ belongs to the boundary $\mathscr{B}$, and write $(\mathbf{x}, \mathbf{t}) \subset \mathscr{B}^{5}$. The simplest examples of boundaries are the subsets of the lattice sites, on a horizontal line or on a vertical line. They correspond to sets with fixed space coordinate, $b(\tau)=(x, \tau)$ or time coordinate, $b(\tau)=(-\tau, t), \tau \in \mathbb{Z}$, respectively.

We will also say that the configuration $(\mathbf{x}, \mathbf{t}) \in \mathscr{D}_{N}$ precedes configuration $\left(\mathbf{x}^{\prime}, \mathbf{t}^{\prime}\right) \in \mathscr{D}_{N}$, and denote $(\mathbf{x}, \mathbf{t}) \preceq\left(\mathbf{x}^{\prime}, \mathbf{t}^{\prime}\right)$, if the sites of $(\mathbf{x}, \mathbf{t})$ are either (weakly) above or (weakly) to the left of the sites of $\left(\mathbf{x}^{\prime}, \mathbf{t}^{\prime}\right)$, i.e. for all $i=1, \ldots, N$ at least one of the inequalities $x_{i} \leq x_{i}^{\prime}, t_{i} \geq t_{i}^{\prime}$ holds. Similarly, one can define the same relation for a configuration $(\mathbf{x}, \mathbf{t}) \in D_{N}$ and a boundary $\mathscr{B}$ and for two boundaries $\mathscr{B}$ and $\mathscr{B} '$ : in the first case $(\mathbf{x}, \mathbf{t}) \preceq \mathscr{B}$ means that the relation $(\mathbf{x}, \mathbf{t}) \preceq\left(\mathbf{x}^{\prime}, \mathbf{t}^{\prime}\right)$ holds for any configuration $\left(\mathbf{x}^{\prime}, \mathbf{t}^{\prime}\right) \subset \mathscr{B}$,

\footnotetext{
${ }^{5}$ Strictly speaking, the sign $\subset$ denoting a subset, is not used quite correctly in this case, since the sites in $(\mathbf{x}, \mathbf{t})$ may coincide. Nevertheless, we will use it to denote the fact that all points $(\mathbf{x}, \mathbf{t})$ are on the boundary $\mathscr{H}$.
} 


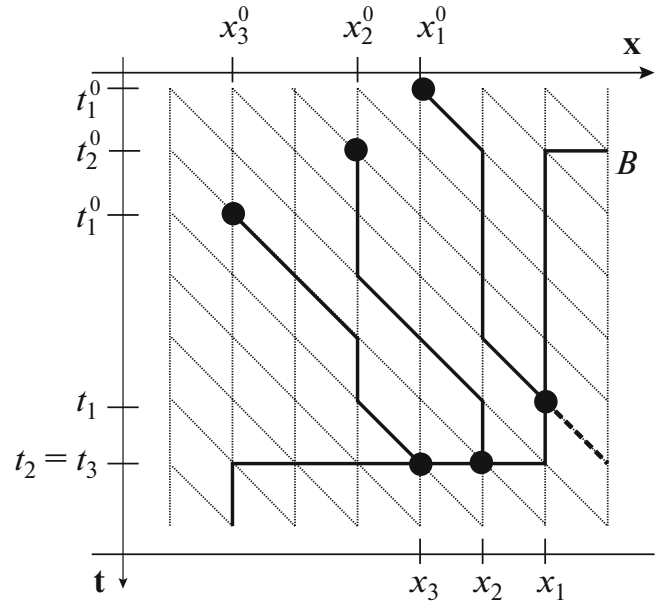

Fig. 3. Transition from initial space-time configuration $\left(\left(x_{1}^{0}, t_{1}^{0}\right),\left(x_{2}^{0}, t_{2}^{0}\right),\left(x_{3}^{0}, t_{3}^{0}\right)\right) \quad$ to $\quad$ a finite configuration $\left(\left(x_{1}, t_{1}\right),\left(x_{2}, t_{2}\right),\left(x_{3}, t_{3}\right)\right)$ on the boundary $\mathscr{B}$, depicted by a black staircase-like line passing from northeast to southwest of the lattice. We consider events in which particles make a mandatory step forward (shown by a dotted line) from the vertices on the vertical sections of the boundary.

and in the second case, $\mathscr{B} \preceq \mathscr{B}$ ', it holds for any pair of configurations $(\mathbf{x}, \mathbf{t}) \subset \mathscr{B}$ and $\left(\mathbf{x}^{\prime}, \mathbf{t}^{\prime}\right) \subset \mathscr{B}$ ' on the boundaries, respectively.

By analogy with the Green function $G_{t}\left(\mathbf{x} \mid \mathbf{x}^{0}\right)$, describing probability of a particle configuration at a fixed moment of time, for any pair of space-time configurations $\left(\mathbf{x}^{0}, \mathbf{t}^{0}\right),(\mathbf{x}, \mathbf{t}) \in \mathscr{D}_{N}$ it is possible to define the generalized Green function $G\left((\mathbf{x}, \mathbf{t})\left(\mathbf{x}^{0}, \mathbf{t}^{0}\right)\right)$ as a partition function of interacting paths pairwise connecting the points of these configurations. It turns out that in the case $q=v=0$ such a partition function also has a determinant form

$$
G\left((\mathbf{x}, \mathbf{t}) \mid\left(\mathbf{x}^{0}, \mathbf{t}^{0}\right)\right)=\operatorname{det}\left\{F_{i-j}\left(x_{i}-x_{j}^{0}, t_{i}-t_{j}^{0}\right)\right\}_{0 \leq i, j \leq N},
$$

where the definition of function $F_{n}(x, t)$ introduced in (42) must be extended to negative times

$$
F_{n}(x, t)=0, \quad t<0 .
$$

This formula gives the probability that the particles having started to walk from sites $\left(x_{1}^{0}, \ldots, x_{N}^{0}\right)$ at times $\left(t_{1}^{0}, \ldots, t_{N}^{0}\right)$ and jumping forward according to the rules of ZRP with particle-wise backward sequential update $^{6}$ will be at the sites $\left(x_{1}, \ldots, x_{N}\right)$ at the times $\left(t_{1}, \ldots, t_{N}\right)$ respectively. In this case, however, it is supposed that if some particle (with number $i$ ) has fallen

\footnotetext{
${ }^{6}$ That is a particle can jump forward with probability $p$ if at the moment there are no particles with lower numbers in the same site or if all such particles also have decided to jump.
}

out of the evolution at the time $t_{i}$, then, from this moment, i.e. for $t \geq t_{i}$, the remaining particles with numbers $j>i$ move like a system of $(N-i)$ particles knowing nothing about the particles fallen out before. In other words, if the next particle will be in the site $x_{i}$ at the moment $t \geq t_{i}$, then the probabilities for it to jump out of the site or stay in the site will be equal to $p$ and $(1-p)$ respectively, regardless of the fact that this site was occupied by another particle before. This condition can be satisfied in a natural way, if we require that the outgoing particle jumps from the site to the next site at its stopping time. Taking into account the space-time ordering of final particle coordinates, this condition ensures an absence of interaction between the particle with number $i$ and particles with numbers $j>i$ at the time moments $t_{j} \geq t \geq t_{i}$.

It is convenient to reformulate the particle exit conditions in terms of traversing a given boundary (Fig. 3). Obviously, for any boundary $B$ and configuration $\left(\mathbf{x}^{0}, \mathbf{t}^{0}\right) \in \mathscr{D}_{N}$, such that $\left(\mathbf{x}^{0}, \mathbf{t}^{0}\right) \preceq \mathscr{B}$, particles will leave the space-time region bounded by boundary $\mathscr{B}$, with probability one. Let us introduce the probability $G^{\mathscr{B}}\left((\mathbf{x}, \mathbf{t}) \mid\left(\mathbf{x}^{0}, \mathbf{t}^{0}\right)\right)$ of positions $(\mathbf{x}, \mathbf{t}) \in \mathscr{B}$ via which the particles exit the space-time region $\{(x, t) \preceq \mathscr{B}\}$, bounded by the boundary $\mathscr{B}$. Particle exit conditions in these terms mean the following. To leave the boundary via a point $b(\tau)=(x, t)$, from which the boundary extends downward, $b(\tau+1)=(x, t+1)$, the particle must jump forward with probability $p$. From a horizontal part of the boundary, for which $b(\tau+1)=(x-1, t)$, both possible outcomes with a total probability of 1 lead to leaving the boundary. So the desired probability of going out of the boundary via configuration $\mathbf{b}=(\mathbf{x}, \mathbf{t})$ has the form

$$
G^{\mathscr{P}}\left(\mathbf{b} \mid \mathbf{b}^{0}\right) \equiv \pi^{\mathscr{P}}(\mathbf{b}) G\left(\mathbf{b} \mid \mathbf{b}^{0}\right),
$$

where $\pi^{\mathscr{P}}(\mathbf{b})=\prod_{i=1}^{N} \pi^{\mathscr{B}}\left(b_{i}\right), b_{i}=\left(x_{i}, t_{i}\right)$ and

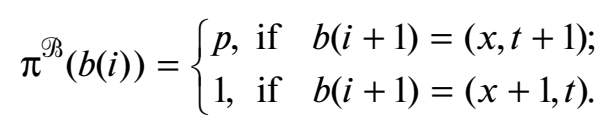

One useful property of the thus defined Green function is the Markov property that generalizes the Markov property of ordinary Green functions: the convolution of two Green functions gives again the Green function. In other words, given if two boundaries $\mathscr{H}^{\prime} \preceq \mathscr{B}$ and two configurations $\mathbf{b}^{0} \preceq \mathscr{B}$ and $\mathbf{b} \subset \mathscr{B}$, the following equality holds

$$
G^{\mathscr{R}}\left(\mathbf{b} \mid \mathbf{b}^{0}\right)=\sum_{\mathbf{b}^{\prime} \in \mathscr{D}_{N}: \mathbf{b}^{\prime} \subset \mathscr{R}^{\prime}} G^{\mathscr{P}^{\prime}}\left(\mathbf{b} \mid \mathbf{b}^{\prime}\right) G^{\mathscr{P}}\left(\mathbf{b}_{+}^{\prime} \mid \mathbf{b}^{0}\right),
$$


where

$$
\left(b_{+}^{\prime}\right)_{i}= \begin{cases}b_{i}^{\prime}, & \text { if } \quad \pi^{\mathscr{P}}\left(b_{i}^{\prime}\right)=1 ; \\ b_{i}^{\prime}+(1,1) & \text { if } \quad \pi^{\mathscr{P}}\left(b_{i}^{\prime}\right)=p .\end{cases}
$$

In conclusion, note that the generalized Green function allows us to study statistics of events taking place not only in different points of space, but also at different times.

\section{DETERMINANTAL PROCESS AND CORRELATION FUNCTIONS}

Although the obtained formulas for the Green functions give the exact probabilities of $N$-particle space-time configurations, they contain a lot of redundant information for the description of physics of the phenomena occurring. From this point of view, it would be more informative to describe the behavior individual particles, in a situation where the total number of particles on the lattice is very large. A method for calculating multipoint space-time distributions of positions of arbitrary number of particles in systems with the infinite number of particles based on the theory of determinantal processes was developed in [75-78]. Here we briefly present the results obtained in $[40,41]$ on the calculation of such distributions for the most general space-time domains, essentially using the concepts of boundaries and generalized Green functions.

We start by considering a system with a finite number of particles $N$ and will take the $N \rightarrow \infty$ limit subsequently. To find the distribution of positions of one particle, one needs to sum the joint distribution of positions of all particles, given by the Green function in the case of a fixed initial configuration, over possible positions of the other particles. Such a summation carried out in [70, 79] allowed obtaining an analogue of formulas known earlier from studies of statistics of Young diagrams and non-decreasing subsequences in generalized permutations [14]. However, another approach that turned out to be more effective is to represent the Green function as a marginal distribution, of another formal distribution of a larger collection of random variables, which, being a determinantal point process, allows a calculation of all multipoint correlation functions at once.

To this end, we first note that given a boundary $\mathscr{B}$, all its vertices $\mathbf{b}=(x, t) \in \mathscr{B}$ can be parameterized by the parameter $\tau=t-x$ that ranges over the set of integers $\mathbb{Z}$. Then the $N$-point configuration $\mathbf{b} \in \mathscr{D}_{N}$ on the boundary, $\mathbf{b} \subset \mathscr{B}$, is associated $N$-tuple of integers $\tau=\left(\tau_{1}, \leq \ldots, \leq \tau_{N}\right)$, such that $b_{i}=\left(x\left(\tau_{i}\right), t\left(\tau_{i}\right)\right)$, and the Green function (46) can be thought of as a probability distribution of such sets.
It turns out that this distribution can be rewritten in terms of the distribution of point configurations on the so-called $N$-boundary $\mathscr{B}=\left(\mathscr{B}_{1}, \ldots, \mathscr{B}_{N}\right)$, which is the disjoint union of $N$ copies of the original boundary $\mathscr{B}$ or, equivalently, on the subsets of $\mathbb{Z} \times\{1, \ldots, N\}$. Consider a signed measure of the form

$$
\begin{aligned}
\mathcal{M}(\mathscr{T}) & =\frac{1}{Z_{N}} \prod_{n=0}^{N-1} \operatorname{det}\left[\phi_{n}\left(\tau_{i}^{n}, \tau_{j}^{n+1}\right)\right]_{i, j=1}^{n+1} \\
& \times \operatorname{det}\left[\Psi_{N i}^{N}\left(\tau_{j}^{N}\right)\right]_{i, j=1}^{N},
\end{aligned}
$$

which assigns probabilities to point sets

$$
\mathscr{T}=\coprod_{1 \leq n \leq N}\left\{\tau_{n}^{n},<\tau_{n-1}^{n},<\ldots,<\tau_{1}^{n}\right\} \subset Z_{\geq \tau_{0}} \times\{1, \ldots, N\} .
$$

Here we have used functions

$$
\phi_{n}(z, y)= \begin{cases}\pi^{\Re_{n+1}}\left(b_{n+1}(y)\right), & y \geq z \\ 0, & y<z\end{cases}
$$

of integers $x, y \in \mathbb{Z}_{\geq \tau_{0}}$, parameterizing points $b_{k}(y) \in \mathscr{H}_{k}, k=1, \ldots, N$ on the corresponding copies of the boundary $\mathscr{B}$ and function

$$
\Psi_{k}^{N}(\tau)=(-1)^{k} \tilde{F}_{-k}\left(b_{N}(\tau)-b_{N k}^{0}\right),
$$

defined by the integral representation (42) for any integer values of the parameter $t \in \mathbb{Z}$, in contrast to the original functions $F_{n}(x, t)$ coinciding with $\tilde{F}_{n}(x, t)$ for $t \geq 0$ and equal to zero for $t<0$. The numbers $\tau_{i}^{j}$ take integer values bounded from below by an integer $\tau_{0}$ selected so that $\Psi_{k}^{N}\left(\tau_{0}\right)=0$. In terms of the boundary $\mathscr{B}$ the value of $\tau_{0}$ is the number of the site $b\left(\tau_{0}\right)$ at the boundary, such that all sites with lower numbers are inaccessible for particles, being located either too far from the initial position in space, or earlier than the departure time. By construction of the boundary, this value always exists. $Z_{N}$ is the normalization constant.

The numbers $\tau_{i}^{n}, i=1, \ldots, n$ are mapped to positions $b_{n}\left(\tau_{i}^{n}\right)$ on $\mathscr{B}_{n}$. Therefore, the measure on point subsets of the $N$-boundary $\mathscr{B}$ is defined as the lift of $M(\mathscr{T})$ under this mapping. Coordinates on the lattice $b_{n}\left(\tau_{j}^{n}\right)$, $1 \leq j \leq n \leq N$, can be considered as coordinates of auxiliary particles numbered by the index $j$ living on the boundary $\mathscr{B}_{n}$. Besides that we introduce the dummy variable $\tau_{n}^{n-1}, 1 \leq n \leq N$, with fixed value $\tau_{n}^{n-1}=\tau_{0}$. For any value $\tau_{i}^{j} \in \mathbb{Z}_{\geq \tau_{0}}$ the following identity holds

$$
\phi_{n}\left(\tau_{n+1}^{n}, \tau_{j}^{n+1}\right) \equiv \pi^{\mathscr{B}_{n+1}}\left(b_{n+1}\left(\tau_{j}^{n+1}\right)\right), \quad j=1, . ., n+1 .
$$


To connect the Green function $G^{\mathscr{P}}\left(\mathbf{b} \mid \mathbf{b}^{0}\right),(46)$, with the measure $M(\mathscr{T})$, (48), the formulas (44), (46) should be rewritten as a sum

$$
\begin{gathered}
G^{\Re}\left(\mathbf{b} \mid \mathbf{b}^{0}\right)=\sum_{A} \prod_{1 \leq i \leq n \leq N} \pi^{\Re_{n}}\left(b_{n}\left(\tau_{i}^{n}\right)\right) \times(-1)^{\frac{N(N-1)}{2}} \\
\times \operatorname{det}\left[\tilde{F}_{-N+1+i}\left(b_{N}\left(\tau_{j+1}^{N}\right)-b_{N i}^{0}\right)\right]_{i, j=0}^{N-1}
\end{gathered}
$$

over the set

$$
A=\left\{\tau_{i}^{j} \in \mathbb{Z}_{\geq \tau_{0}}, 2 \leq i \leq j \leq N \mid \tau_{i}^{j} \geq \tau_{i}^{j-1}, \tau_{i}^{j}>\tau_{i+1}^{j+1}\right\} .
$$

To calculate the sum in each row of the matrix under the determinant, we notice that the functions $\tilde{F}_{n}(x, t)$ satisfy the following adjacency relations

$$
\begin{gathered}
\tilde{F}_{n}(x, t)=\tilde{F}_{n+1}(x-1, t)-\tilde{F}_{n+1}(x, t), \\
p \tilde{F}_{n}(x, t)=\tilde{F}_{n+1}(x, t+1)-\tilde{F}_{n+1}(x, t),
\end{gathered}
$$

which can be written in terms of coordinates at the boundaries as a single equation

$$
\pi^{\Re}(b(\tau)) \tilde{F}_{n}(b(\tau))=\tilde{F}_{n+1}(b(\tau+1))-\tilde{F}_{n+1}(b(\tau)) .
$$

Using this equation, we see that each summation decreases the subscript in functions $\tilde{F}_{n}$ by one, and the boundary terms are canceled with the next row in all summations except for the the last, in which the boundary terms vanish due to the choice of $\tau_{0}$. The last step is to make sure that the summation in (53) can be extended to a wider set

$$
\tilde{A}=\left\{\tau_{i}^{j} \in \mathbb{Z}_{\geq \tau_{0}}, 2 \leq i \leq j \leq N \mid \tau_{i}^{j}<\tau_{i-1}^{j}\right\},
$$

by requiring the summands to coincide with the summands of (53) in $A \cap \tilde{A}$ and to vanish in $\tilde{A} \backslash A$. This requirement is ensured by the product of determinants in (48). As a result, the statement about the connection between the Green function and the measure $M(\mathscr{T})$ has the form

$$
\mathscr{G}^{\mathscr{P}}\left(\mathbf{b} \mid \mathbf{b}^{0}\right)=\mathcal{M}\left(\bigcup_{k=1}^{N}\left\{\tau_{1}^{k}=\tau_{k}\right\}\right) .
$$

The key point that makes the transition to the measure $M(\mathscr{T})$ remarkably effective is an observation that the measure $\mathcal{M}(\mathscr{T})$ is determinantal. This means that for any $n \in \mathbb{N}$ the $n$-point correlation function

$$
\rho_{n}\left(a_{1}, \ldots, a_{n}\right)=\mathcal{M}\left(\left(a_{1}, \ldots, a_{n}\right) \subset \mathscr{T}\right),
$$

defined as the probability that a random point configuration $\mathscr{T}$ contains a subset $a=\left(a_{1}, \ldots, a_{n}\right)$, is given by the determinant of a matrix $n \times n$

$$
\rho_{n}\left(a_{1}, \ldots, a_{n}\right)=\operatorname{det}\left\{K\left(a_{i}, a_{j}\right)\right\}_{1 \leq i, j \leq n},
$$

whose matrix elements are expressed in terms of the unique function $K\left(a, a^{\prime}\right)$ of two variables on $\tilde{A} \times \tilde{A}$, called the correlation kernel.

We are interested in the probabilities characterizing what is happening in given subsets of $\tilde{A}$. The simplest of these probabilities is the emptiness formation probability, i.e. the probability that a random point configuration $\mathscr{T}$ does not contain points of the set $a \subset \tilde{A}$. Using the exclusion-inclusion principle, this probability can be expressed in terms of the correlation functions

$$
\begin{gathered}
M(a \cap \mathcal{T}=\varnothing)=1-\sum_{a_{i} \in a} \rho_{1}\left(a_{1}\right) \\
+\sum_{a_{1} \neq a_{2} \in a} \rho_{2}\left(a_{1}, a_{2}\right)-\sum_{a_{1} \neq a_{2} \neq a_{3} \in a} \rho_{2}\left(a_{1}, a_{2}\right)+\ldots
\end{gathered}
$$

Substituting for the correlation functions their determinant form (57), we notice that the infinite sum turns into the Fredholm determinant of an operator with kernel $K(x, y)$ acting on functions on $\tilde{A}$,

$$
M(a \cap \mathscr{T}=\varnothing)=\operatorname{det}(1-K)_{l^{2}(a)} .
$$

The assertion about the measure $\mathcal{M}(\mathscr{T})$ being determinantal is known as the Eynard-Meta theorem [30]. It can be justified, using arguments based on the Theorem 1, which also allows one to deduce an explicit expression for the kernel [31, 76]. To this end, we introduce convolutions

$$
\begin{aligned}
& \phi^{\left(n_{1}, n_{2}\right)}(x, y) \\
& =\left\{\begin{array}{lc}
\left(\phi_{n_{1}} * \phi_{n_{1}+1} * \ldots * \phi_{n_{2}-1}\right)(x, y), \quad n_{1}<n_{2} \\
0, & n_{1} \geq n_{2}
\end{array},\right.
\end{aligned}
$$

where $(a * b)(x, y)=\sum_{z \in \mathbf{Z}_{\tau_{0}}} a(x, z) b(z, y)$, and

$$
\Psi_{n-j}^{n}(\tau)=\left(\varphi^{n, N} * \Psi_{N-j}^{N}\right)(\tau)
$$

Point configurations $\mathcal{T}$ can be thought of as sets of coordinates of auxiliary particles, and each determinant in the formula (48) - as a partition function of the non-intersecting paths in the sense of KMLGV theorem. Then the product in (48) will describe the following picture (Fig. 3). First, the paths of $N$ particles propagate without intersections through the twodimensional lattice until they reach the boundary $\mathscr{B}$, interpreted as $\mathscr{B}_{N}$. Then, all the particles pass, maintaining the order, from sites of $\mathscr{B}_{N}$ to sites of $\mathscr{B}_{N-1}$, and the first particle goes to the position with the number $\tau_{N}^{N-1}=\tau_{0}$. The other particles jump to the next boundary $\mathscr{B}_{N-2}$, including the second particle that reaches $\tau_{N-2}^{N-1}=\tau_{0}$. The remaining $N-2$ particles go to $\mathscr{S}_{N-3}$ and so on, until each particle goes to position $\tau_{0}$ along the corresponding boundary, ending with the last particle going to the point with the number $\tau_{0}$ of the fictitious boundary $\mathscr{B}_{0}$, consisting of this single site. So the function $\phi_{n}(z, y)$ will play the role of the weight of the transition between sites $b_{n+1}(y)$ and $b_{n}(x)$ of two adjacent boundaries $\mathscr{S}_{n+1}$ and $\mathscr{B}_{n}$. Accordingly, 
$\phi^{\left(n_{1}, n_{2}\right)}(x, y)$ will have the meaning of the weight of transition between sites of boundaries $\mathscr{B}_{n_{1}}$ and $\mathscr{B}_{n_{2}}$, parameterized by numbers $x$ and $y$ in (59). In turn, the argument of the function $\Psi_{n-j}^{n}(\tau)$ parameterizes sites of the boundary $\mathscr{B}_{n}$, and the function (60) itself plays the role of the weight of transition from the starting point $b_{j}^{0}$ to the site $b(\tau)$ of the boundary $\mathscr{S}_{n}$. In this picture the $N$-particle configuration of our original process corresponds to $N$ positions of the particle with the number 1 on each of the $N$ boundaries.

Let us fix $1 \leq k \leq N$ different integers $\left(1 \leq n_{1}<n_{2}<\ldots n_{k}<N\right)$ and a set of $s=s_{1}+\ldots+s_{k}$ sites on the corresponding boundaries with numbers $\tau=\left\{\left(\tau_{1}^{n_{i}}<\cdots<\tau_{s_{i}}^{n_{i}}\right)\right\}_{i=1}^{k}$.

Our task is to calculate the correlation function for the measure $\mathcal{M}$, which, in terms of paths, is the probability that our paths will pass through points from the set $\tau$. Before moving on to calculate this probability, we will try to calculate the normalization coefficient $Z_{N}$ of the measure $\mathcal{M}$, or the partition function of the ensemble of nonintersecting paths. According to the KMLGV theorem, it is given by the determinant of the matrix

$$
Z_{N}=\operatorname{det} M
$$

whose matrix elements

$$
M_{i j}=\sum_{\tau} \phi_{j-1}\left(\tau_{j}^{j-1}, \tau\right) \Psi_{j i}^{j}(\tau), \quad 1 \leq i, \quad j \leq N
$$

are given by the weights of transitions from the starting points $\mathbf{b}^{0}=\left(b_{1}^{0}, \ldots, b_{N}^{0}\right) \quad$ to endpoints $\mathbf{b}\left(\tau_{0}\right)=$ $\left(b^{N-1}\left(\tau_{0}\right), \ldots, b^{0}\left(\tau_{0}\right)\right)$ in positions with number $\tau_{0}$ on each of the boundaries $\mathscr{B}_{N-1}, \ldots, \mathscr{B}_{0}$ respectively.

Now, to calculate the required probability, we add to the sets of initial and end points of paths extra sites of the boundaries with numbers from the set $\tau$ (Fig. 4), and calculate the partition function of nonintersecting paths from $\left(\mathbf{b}^{0}, \mathbf{b}(\tau)\right)$ in $\left(\mathbf{b}\left(\tau_{0}\right), \mathbf{b}(\tau)\right)$, where by $\mathbf{b}(\tau)$ in both brackets we mean the set of boundary sites corresponding to numbers from the set $\tau$. Up to a sign, such a partition function is given by the determinant of the block matrix of the form

$$
\tilde{M}=\left(\begin{array}{l}
{\left[\mathbf{b}^{0} \rightarrow \mathbf{b}\left(\tau_{0}\right)\right]\left[\mathbf{b}(\tau) \rightarrow \mathbf{b}\left(\tau_{0}\right)\right]} \\
{\left[\mathbf{b}^{0} \rightarrow \mathbf{b}(\tau)\right][\mathbf{b}(\tau) \rightarrow \mathbf{b}(\tau)]}
\end{array}\right),
$$

where matrix elements of blocks are given by weights of transitions between sites from the sets in square brackets. Accordingly, we have

$$
\left[\mathbf{b}^{0} \rightarrow \mathbf{b}\left(\tau_{0}\right)\right]=M
$$

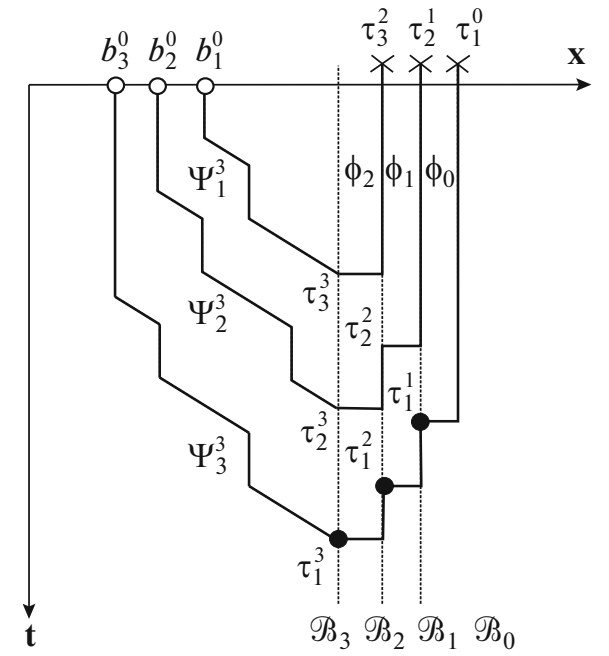

Fig. 4. Determinantal process in terms of nonintersecting paths for $N=3$-particle system. Copies $\mathscr{B}_{1}, \ldots, \mathscr{B}_{3}$ of boundary $\mathscr{B}$ are shown by vertical dotted lines, and the boundary $\mathscr{B}_{0}$ consists of a single point with number $\tau_{1}^{0}=\tau_{0}$. The paths connecting points of the initial configuration $b_{1}^{0}, \ldots, b_{3}^{0}$ with points $\tau_{1}^{3}, \ldots \tau_{3}^{3}$ of the boundary $\mathscr{S}_{3}$ have weights $\Psi_{1}^{3}, \ldots, \Psi_{3}^{3}$ respectively. The transition weight between vertices with numbers $\tau_{1}^{i}, \tau_{2}^{i+1}$ of adjacent

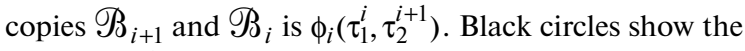
positions of the particles in the original process.

is the initial matrix (61). Also

$$
\left[\mathbf{b}^{0} \rightarrow \mathbf{b}(\tau)\right]_{i,(n, j)}=\Psi_{n i}^{n}\left(\tau_{j}^{n}\right),
$$

where the second matrix index consists of two components, since the elements of the set $\tau$ are numbered by two numbers, the number of the boundary $n$ and the number of the point on the boundary $j$. Weights of transitions between points of the set $\tau$ have the form

$$
[\mathbf{b}(\tau) \rightarrow \mathbf{b}(\tau)]_{\left(x, n_{1}\right),\left(y, n_{2}\right)}=\phi^{n_{1}, n_{2}}(x, y) .
$$

Finally, the transition from a point with the number $\tau_{j}^{n}$ at the boundary $\mathscr{B}_{n}$ to the point with the number $\tau_{0}$ of the boundary $l=0, \ldots, N-1$ is given by convolution

$$
\left[\mathbf{b}(\tau) \rightarrow \mathbf{b}\left(\tau_{0}\right)\right]_{(n, j), l+1}=\left(\phi_{l} * \phi^{l+1, n}\right)\left(\tau_{0}, \tau_{j}^{n}\right) .
$$

In principle, there are different options for constructing nonintersecting paths from $\left(\mathbf{b}^{0}, \mathbf{b}(\tau)\right)$ to $\left(\mathbf{b}\left(\tau_{0}\right), \mathbf{b}(\tau)\right)$. Therefore, as follows from the theorem 1, the determinant of the matrix $\tilde{M}$ is equal to the signed sum over all the possibilities in r.h.s. of the formula (5). However, after looking carefully at possible transitions, one can see that all nonzero terms correspond to permutations whose parity is equal to the parity of 


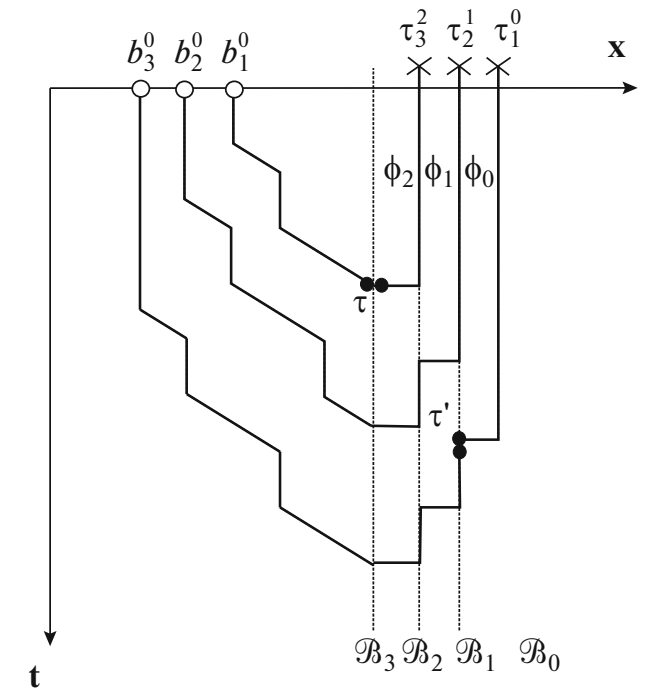

Fig. 5. Paths on the graph used to calculate the two-point correlation function $\rho_{2}\left((\tau, 3),\left(\left(\tau^{\prime}, 1\right)\right)\right)$. The calculation consists in finding the (normalized) partition function of paths passing through the points $\tau, \tau^{\prime}$ on the boundaries. $\mathscr{B}_{3}$ and $\mathscr{B}_{1}$, respectively. To do this, we fix additional pairs of start and end points (black circles).

$s=|\tau|$, the number of points in the set $\tau$. Then the required probability has the form

$$
M(\tau \subset \mathscr{T})=(-1)^{s} \frac{\operatorname{det} \tilde{M}}{\operatorname{det} M},
$$

whence, using the formula for the determinant of the block matrix

$$
\operatorname{det}\left(\begin{array}{ll}
A & B \\
C & D
\end{array}\right)=\operatorname{det} A \operatorname{det}\left(D-C A^{-1} B\right)
$$

we get

Theorem 4. (Eynard-Meta [30], Borodin-Rains [31])

$$
\mathcal{M}(\tau \subset \mathcal{T})=\operatorname{det}_{\substack{1 \leq m, l \leq k \\ 1 \leq i_{n}, j_{n} \leq r_{n}, n=1, \ldots, k}}\left\{K\left(n_{k}, \tau_{i_{m}}^{n_{m}} ; n_{l}, \tau_{j_{l}}^{n_{l}}\right)\right\} .
$$

Here

$$
\begin{gathered}
K\left(n_{1}, x ; n_{2}, y\right)=-\phi^{\left(n_{1}, n_{2}\right)}(x, y) \\
+\sum_{1 \leq i, j \leq N} \Psi_{n_{1}-i}^{n_{1}}(x) M_{i j}^{-1}\left(\phi_{j-1} * \phi^{j, n_{2}}\right)\left(\tau_{0}, y\right),
\end{gathered}
$$

where, in a contrast to the definition (59), convolution under the sum in the case of $j=n_{2}$ is understood as $\left(\phi_{n_{2}-1} * \phi^{j, n_{2}}\right)\left(\tau_{0}, y\right)=\phi_{n_{2}-1}\left(\tau_{0}, y\right)$ and $\left(\phi_{n_{2}-1} * \phi^{j, n_{2}}\right) \times$ $\left(\tau_{0}, y\right)=0$ for $j>n_{2}$. Therefore, only the terms with $1 \leq j \leq n_{2}$ contribute to the sum over $j$.
This formula is complicated because the inversion of the matrix $M$ is not an easy task. Note, however, that the convolution of the last factor under the sum with the function $\Psi_{n-j}^{n}(x)$ gives the matrix element

$$
M_{j i}=\sum_{x \in Z_{z_{0}}}\left(\phi_{j-1} * \phi^{j, n}\right)\left(\tau_{0}, y\right) \Psi_{n-j}^{n}(y),
$$

for $j<n \leq N$ and since $\sum_{i=1}^{N} M_{k i}^{-1} M_{i j}=\delta_{k j}$, function

$$
\Phi_{n-i}^{n}(x)=\sum_{j=1}^{n_{2}-1} M_{i j}^{-1}\left(\phi_{j-1} * \phi^{j, n}\right)\left(\tau_{0}, x\right)
$$

satisfies the orthogonality relation

$$
\sum_{\tau \in \mathbb{Z}_{\geq \tau_{0}}} \Phi_{i}^{n}(\tau) \Psi_{j}^{n}(\tau)=\delta_{i, j}
$$

and can be constructed using the Gram-Schmidt orthogonalization process.

Corollary 5. Consider the system of functions

$$
\begin{aligned}
& \left\{\left(\phi_{0} * \varphi^{(1, n)}\right)\left(\tau_{1}^{0}, \tau\right), \ldots,\left(\phi_{n-2} * \phi^{n-1, n}\right)\right. \\
& \left.\quad \times\left(\tau_{n-1}^{n-2}, \tau\right), \phi_{n-1}\left(\tau_{n}^{n-1}, \tau\right)\right\} .
\end{aligned}
$$

which, being linearly independent, form a basis in the $n$-dimensional linear space $V_{n}$. Using the orthogonality relations (62), construct another basis $\left\{\Phi_{j}^{n}(\tau), j=0, \ldots, n-1\right\}$ of $V_{n}$. If $\phi_{n}\left(\tau_{n+1}^{n}, \tau\right)=c_{n} \Phi_{0}^{n}(\tau)$ with some $c_{n} \neq 0$, $n=1, \ldots, N$, the kernel will look as follows

$$
\begin{gathered}
K\left(n_{1}, \tau_{1} ; n_{2}, \tau_{2}\right)=-\mathrm{l}^{\left(n_{1}, n_{2}\right)}\left(\tau_{1}, \tau_{2}\right) \\
+\sum_{k=1}^{n_{2}} \Psi_{n_{1}-k}^{n_{1}}\left(\tau_{1}\right) \Phi_{n_{2}-k}^{n_{2}}\left(\tau_{2}\right) .
\end{gathered}
$$

Thus, the problem of constructing the correlation kernel is reduced to the problem of constructing functions $\Phi_{i}^{n}(\tau)$, orthogonal to functions $\Psi_{j}^{n}(\tau)$. It can be solved explicitly for several special types of initial conditions. In particular, we present the results for the case of all particles initially being in one site

$$
\mathbf{b}^{0}=((0,0), \ldots,(0,0)) .
$$

Note that in the case of ZRP with backward sequential update, which we are considering, the motion of the first $N$ particles does not depend on particles with larger numbers. Therefore, the results obtained can be considered as the statistics of the first $N$ particles in the system, where initially one site con- 
tains an infinite number of particles. Omitting details we will give the answer for the kernel

$$
\begin{gathered}
K\left(n_{1}, \tau ; n_{2}, \tau_{2}\right)=\oint_{\Gamma_{1}} \frac{d V}{2 \pi \mathrm{i} V} \oint_{\Gamma_{0}, v} \frac{d w}{2 \pi \mathrm{i} w} \\
\quad \frac{\left(1-p\left(\frac{w-1}{w}\right)\right)^{t\left(\tau_{1}\right)}}{\left(1-p\left(\frac{v-1}{V}\right)\right)^{t\left(\tau_{2}\right)} \frac{(w-1)^{n_{1}}}{(V-1)^{n_{2}}} \frac{w^{x\left(\tau_{1}\right)}}{V^{x\left(\tau_{1}\right)}}} \\
-1\left(n_{2}>n_{2}\right) \oint_{\Gamma_{0}, 1} \frac{d w}{2 \pi \mathrm{i} w} \frac{\left(1-p+1 / \pi_{2}-1\right)}{\left.\left(\frac{w-1}{w}\right)\right)^{t\left(\tau_{1}\right)-t\left(\tau_{2}\right)} w^{x\left(\tau_{1}\right)-x\left(\tau_{2}\right)}}
\end{gathered}
$$

where $\pi_{2} \equiv \pi^{\Re}\left(b\left(\tau_{2}\right)\right)$, and the integration is performed along closed contours $\Gamma_{\omega}$, where $\omega$ is a set of singularities of the integrand that should fall inside the contour.

To return to the statistics of the positions of particles in ZRP, one can use the Fredholm determinant expression (58) of the emptiness formation probability. Taking as a subset $a$ the union of half-axes

$$
a=\left\{\mathscr{T} \bigcup_{i=1}^{k}\left\{\tau_{1}^{n_{i}}>a_{k}\right\}\right\}
$$

we obtain the distribution of points $\mathbf{b}=\left(b_{1}\left(\tau_{1}^{1}\right), \ldots, b_{N}\left(\tau_{1}^{N}\right)\right)$ of exits from the boundary in the ZRP for particles with numbers $n_{1}, \ldots, n_{k}$,

$$
\begin{gathered}
\left.\mathbb{P}\left(\mathbf{b} \mid\left(\tau_{n_{1}} \leq a_{1}\right\} \cap \ldots \cap\left\{\tau_{n_{k}} \leq a_{k}\right\}\right)\right) \\
\left.=\operatorname{det}\left(1-\chi_{a} K \chi_{a}\right)\right)_{l^{2}\left(\left\{n_{1}, \ldots, n_{m}\right\} \times \mathbb{Z}\right)},
\end{gathered}
$$

where $\chi_{a}\left(x_{1}, \ldots, x_{k}\right)=\prod_{i=1}^{k} \rrbracket_{x>a_{i}}$ are projectors onto semi-infinite subsets of integers.

We add to the said in this section that this result can be generalized from one boundary to an arbitrary sequence of nested boundaries. Just like for one boundary we started with the formula for the Green function, from which we passed to the sum over the coordinates of the auxiliary particles, to consider multiple boundaries, one needs to start with a convolution of several Green functions along these boundaries, similar to the convolution in the formula (47). The convolution turns into an extended sum over auxiliary variables, having the form of a marginal distribution of a determinant process. As a result the joint particle position distribution on the boundaries, where the particle numbers strictly increase within one boundary and weakly increase when passing between two adjacent boundaries, can be expressed in the form of Fredholm determinants. This extension allows one to get rid of the time ordering of exits, so that the only remaining restriction is the requirement of weak ordering of spatial coordinates. The formula (65) for the correlation kernel remains unchanged, except for the presence of an implicit dependence of the space coordinates $x_{i}(\tau)$ and time coordinates $t_{i}(\tau)$ as functions of $\tau$ on the form of the boundary on which these coordinates live.

\section{ASYMPTOTIC ANALYSIS: LIMITING HYDRODYNAMICS AND UNIVERSAL FLUCTUATIONS}

As discussed in the introduction, the behavior of a thermodynamic system on a large scale can be characterized by typical values of thermodynamic quantities and their universal fluctuations around these values. As shown, in the absence of long correlations the scenario for this behavior is quite simple. There are two characteristic scales, thermodynamical and fluctuational, the behavior in which is governed by LLN and CLT, respectively.

Unlike systems in the state of thermodynamic equilibrium, the nonequilibrium systems have flows carrying information about fluctuations between different parts of the system, which can significantly affect the system behavior on the large scale, leading to the emergence of new characteristic scales and universal distributions. One example of non-equilibrium universal behavior is provided by systems that are unified into the KPZ universality class. Historically the notion of this class arose from studies of scaling behavior of the random growing interfaces, such as boundaries between different phases of matter, combustion, drying, wetting, crystallization fronts, bacterial colonies, etc. In general, it is assumed that this class combines elastic surfaces or lines under the action of a random force with short range correlations.

In the theory, the shape of a growing interface is described by the height $h_{t}(x)$, the function of spatial coordinates $x$ parameterizing the points of the substrate, and time $t$. The scaling behavior of the height function in systems of KPZ universality class is characterized by two independent critical exponents that can be selected for example as the roughness exponent $\zeta$ and dynamic exponent $z$. For example, in a system of linear size $L$ this is manifested in the fact that at large time, $t \gg t_{c}$, the width $w_{t}=\sqrt{\mathbb{E}\left(h_{t}(x)^{2}-\bar{h}_{t}^{2}\right)}$ of initially flat interface, $w_{0}=0$, saturates to values of order of $L^{\zeta}$, while at small time, $t \ll t_{c}$, it does not depend on $L$, growing with time according to a power law. Here $\bar{h}_{t}$ is the spatial average of the interface height. The transition occurring at the characteristic times $t_{c} \asymp L^{z}$ is called roughening. The two asymptotic regimes can be combined within a single FamilyVicek scaling ansatz [80]

$$
w_{t}=L^{\zeta} \Phi\left(\frac{t}{L^{z}}\right)
$$


where $\Phi(x)$ is a scaling function with asymptotics $\Phi(x) \asymp x^{1 / z}, x \rightarrow 0$ and $\Phi(x) \rightarrow$ const, $x \rightarrow \infty$. In the infinite system, instead of $\zeta$ and $z$ it is more convenient to use another pair of critical exponents $\alpha=\zeta / z$ and $\beta=1 / z$, responsible for the power-law growth of fluctuations $h_{t}-\bar{h}_{t} \asymp t^{\alpha}$ and the dynamic correlation length $\xi(t) \asymp t^{\beta}$, which specifies the characteristic distances at which different parts of the interface are non-trivially correlated.

An attempt to find a minimal and most universal model belonging to the KPZ universality class resulted in appearance of the eponymous equation [4]

$$
\frac{\partial h}{\partial t}=v \frac{\partial^{2} h}{\partial x^{2}}+\frac{\lambda}{2}\left(\frac{\partial h}{\partial x}\right)^{2}+\eta(x, t) .
$$

Here $h(x, t)$ is the height of the growing interface, and $\eta(x, t)$ is white noise, i.e. random Gaussian field with zero mean

$$
\mathbb{E} \eta(x, t)=0,
$$

and covariance

$$
\mathbb{E} \eta(x, t) \eta\left(x^{\prime}, t^{\prime}\right)=D \delta\left(x-x^{\prime}\right) \delta\left(t-t^{\prime}\right),
$$

and $\lambda, v$ and $D$ are the dimensional parameters of the problem. Exact critical exponents $\zeta$ and $z$ describing the scaling behavior of solutions of this equation in the case of space-time of dimension $d=1+1$ were found already in [4] using arguments based on symmetries of this equation and on the renormalization group. The value $\zeta=1 / 2$ reflects the fact that the stationary solution in the infinite system is simply a Brownian motion $h_{s t}(x)=\sqrt{A} B_{x}$ with amplitude

$$
A=D /(2 v),
$$

and in particular

$$
\mathbb{V}\left(h_{s t}(x)-h_{s t}(y)\right)=A|x-y|,
$$

where $\mathbb{V}(\xi)$ is the variance of the random variable $\xi$. In non-stationary regime the statistics of the solution to Eq. (66) demonstrates a richer scaling behavior, the general form of which can, however, be predicted from simple dimensional analysis. It is expected that at large times, $t \rightarrow \infty$, height fluctuations grow around the mean with time, measured in units proportional to $\left(|\lambda| A^{2}\right)^{-1}$, according to power law with exponent $\alpha$. We write this down through the following scaling hypothesis

$$
h_{t}(x)-\mathbb{E} h_{t}(x)=\left(\frac{|\lambda| A^{2}}{2} t\right)^{\alpha} \mathscr{X}+o(t),
$$

where the factor of two in the denominator is added for the brevity of further formulas. The result of dividing the deviation of the height function from its mean by the corresponding scaling (dimensional) factor gives a dimensionless random variable $\mathscr{X}$, which is expected to be universal within the KPZ universality class. As the recent history of the theory development shows, the distribution of this quantity still depends on the global shape of initial conditions that survives in the scaling limit. However it turns out to be insensitive to microscopic details of the interface dynamics. The random variable $\mathscr{X}$ still depends on the coordinate $x$. If at large time we consider a spatial scale, measuring the distance in units of dynamical correlation length

$$
\xi(t)=\frac{1}{A}\left(\frac{|\lambda| A^{2}}{2} t\right)^{\beta}
$$

we will obtain a one-parameter family of random variables $\mathscr{L}(u)$, expected to play a role of the universal within the KPZ theory random process. Depending on particular initial conditions the one-point distribution of $\mathscr{L}(u)$ may or may not depend on $u$, but for different values of the parameter $u$ these random variables turn out to be nontrivially correlated with each other. To fully characterize the random process $\mathscr{X}(u)$ one has to find all joint distributions of the variables $\left(\mathscr{L}\left(u_{1}\right), \ldots, \mathscr{X}\left(u_{k}\right)\right)$ for all $k \in \mathbb{N}$. A more general question also concerns joint distributions of the interface height function at different points in space-time plane. Similar universal random processes also arise when one considers the scaling limits of the height function (or other observables) along paths on the space-time plane.

The solution to the KPZ equation in the sense of finding all multipoint distributions is not an easy task that has not been solved yet completely. However, all that has been said regarding the scaling behavior of its solutions can be equally attributed to other models in the same universality class. The search for the universal distributions and processes served as a motivation for finding exact solutions for systems of interacting particles. In our case, we expect that the scaling limit of multipoint distributions obtained in the previous subsection will also be universal. To formulate these statements, we first discuss the scaling hypotheses about the structure of the interface dynamics associated with our system of particles on a large scale.

To compare our results with scaling predictions for the growing interface described by the KPZ equation, we associate particle configurations $\mathbf{n}(t)=\left\{n_{i}(t)\right\}_{i \in L}$ on the integer lattice $\mathscr{L}=\mathbb{Z}$ with nonincreasing staircase-like height function $h_{t}(x) \in \mathbb{Z}$ of $x \in \mathbb{R}$, taking only integer values that change abruptly, decreasing by $n_{i}(t)$ at integer values $x=i$, i.e.

$$
h_{t}(i+0)-h_{t}(i)=n_{i}(t), \quad \forall i \in \mathbb{Z},
$$

see Fig. 6. Having set the values of height function at one point, one can uniquely restore the entire function from this relation. For example, for configurations bounded on the right, where $n_{i}(t)=0$ for large enough 
$i$, we can assume that $\lim _{x \rightarrow \infty} h_{t}(x)=0$. Then at the initial moment of the evolution considered above, when the infinite number of particles is in the site $i=0$,

$$
h_{0}(x)= \begin{cases}\infty & x \leq 0 \\ 0 & x>0\end{cases}
$$

the interface consists of two rays going from one point at right angle to each other. Further evolution of the interface follows from the particle evolution rules. At each step of time we go through the whole system from right to left and add $m_{i-1}$ unit squares into each internal corner in position $i$ with probability $\varphi\left(m_{i-1} \mid n_{i-1}\right)$,

$$
h_{t+1}(x)=h_{t}(x)+m_{i-1}, \quad x \in[i-1, i) .
$$

The fact that $0 \leq m_{i-1} \leq n_{i-1}$ preserves the height function non-increasing. In particular, in the case $v=0$, we consider, each next square at a given position is added with a probability $p$, until either the addition stops with probability $(1-p)$ or the height reaches the height at the previous unit half-interval.

What does the typical shape of such an interface look like on the large scale at large time? It turns out that similarly to the equilibrium situation there are two characteristic scales in the system responsible for the limiting shape (LLN) and typical fluctuations (CLT). On the LLN scale, $x \sim t \rightarrow \infty$, the typical interface shape is governed by the Euler hydrodynamics of the particle system, which is dictated by the conservation laws of the system. In our case the only conservation law is the particle conservation, which assumes that the density $\rho(x)$ of particles on the lattice satisfies the continuity equation

$$
\partial_{t} \rho(x, t)+\partial_{x} j(x, t)=0
$$

Here $j(x, t)$ is the particle current through the point $x$ at the time $t$. The key fact that allows us to make this equation closed is the local quasi-stationarity hypothesis. It asserts that on a large scale the nonstationary particle system behaves locally like the infinite stationary translation-invariant system with the density of particles equal to the local density in the nonstationary system. In particular this suggests that the current $j(x, t)$ depends on coordinates and time only through the dependence on the density $\rho(x, t)$, and the form of this dependence is given by the relation between the current and the density of particles in the infinite system with a translation-invariant stationary state,

$$
j(x, t)=j_{s t}(\rho(x, t)) .
$$

In what follows, we will assume that $j_{s t}(\rho)$ is a convex function for $\rho>0$ and $j_{s t}(0)=0$. As a result, we obtain an equation for the density

$$
\partial_{t} \rho+j_{\mathrm{st}}^{\prime}(\rho) \partial_{x} \rho=0
$$

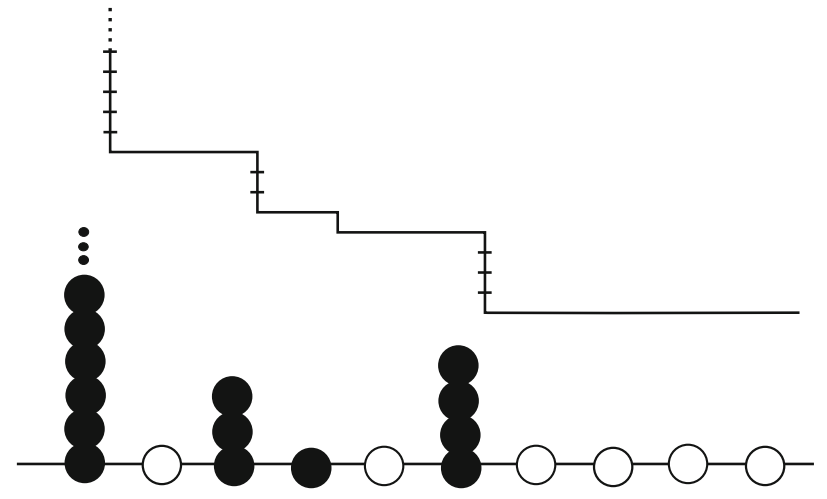

Fig. 6. Correspondence of particle configuration and growing interface. The example in the figure shows a configuration in which the leftmost site contains infinitely many particles. All sites to the right of the ones shown are supposed to be empty, then the parts of the interface remaining outside the figure are two rays going right and up at right angle.

which is solved by the method of characteristics. For the initial condition $\rho(x, 0)=\infty \cdot I_{x \leq 0}$, all characteristics are rays in the $x-t$ plane, outgoing from origin $(x, t)=(0,0)$ into the first quadrant. The solution for the density function $\rho(x, t)=\rho(\chi)$ is the function of variable $\chi=x / t$ obtained by inverting relation

$$
j_{\text {st }}^{\prime}(\rho)=\chi
$$

in the domain $0<\chi<j_{\infty}^{\prime}(0)$ and

$$
\rho(\chi)=0,
$$

for $\chi \geq j_{\infty}^{\prime}(0)$.

To relate this solution to the position $x_{n}(t)$ of the particle with number $n$ at time $t$, note that exactly $n$ particles are weakly to the right of the site $x_{n}(t)$. Suppose that the position of the particle is a function $x_{n}(t)=t \chi(\theta)$ of the ratio $\theta=n / t$. Then

$$
\begin{gathered}
\theta=\int_{\chi}^{j_{\mathrm{st}}^{\prime}(0)} \rho(y) d y=-\chi \rho-\int_{\chi}^{j_{\mathrm{st}}(0)} y \rho^{\prime}(y) d y \\
=-\chi \rho+j_{s t}(\rho),
\end{gathered}
$$

where we used the change of variables $y \rightarrow \rho(y)$ given by the relation (72) and integration by parts. Comparing Eqs. (72) and (73), we conclude what

$$
\theta(\chi)=\inf _{\rho \in(0, \infty)}\left(-\chi \rho+j_{s t}(\rho)\right)
$$

is the minus Legendre transform of the function $j(\rho)$. Since the height of the associated interface at position $x=\chi t$ is nothing but the number of particles that have 
passed through this point, $\theta(\chi)$ is nothing but its limiting shape, which appears in the analogue of the LLN

$$
\lim _{t \rightarrow \infty} \frac{h_{t}(\chi t)}{t}=\theta(\chi)
$$

Conversely, since for a nonzero density $\theta(\chi)$ is the monotone function, $d \theta / d \chi=-\rho<0$, it can be inverted in the region $0<\chi<j_{\infty}^{\prime}(0)$, so that as a result we obtain the function $\chi(\theta)$, which appears in the analogue of the LLN for $x_{n}(t)$ :

$$
\lim _{t \rightarrow \infty} \frac{x_{\theta t}(t)}{t}=\chi(\theta) .
$$

Here, since the arguments used are purely heuristic, we do not make statements about the type of convergence of random variables. Existing proofs for specific examples assume the almost sure convergence.

To obtain explicit formulas for functions connecting $\rho, \chi$ and $\theta$ for ZRP with hopping probabilities (28), we will use the fact that in the stationary state of the infinite system the occupation numbers are independent, and the one-site distribution has the form (32),

$$
\mathbb{P}_{s t}\left(n_{i}=k\right)=\frac{z^{k} \mathrm{f}(k)}{\jmath(z)},
$$

where the stationary weight $\mathrm{f}(k)=\sum_{l=0}^{k} v(l) w(k-l)$ is the convolution of two sets of parameters $v(l)$ and $w(l)$, the products of which determine the hopping probabilities $\varphi(m \mid n)=v(m) w(n-m) / \mathrm{f}(n), z$ is the activity, the parameter of the distribution which fixes the average density of particles, and $z(z)$ is a one-site partition function defined as the generating function of stationary weights $z(z)=\sum_{n \geq 0} z^{n} \mathrm{f}(n)$. The connection between the average particle density and activity is given by the standard thermodynamic relation

$$
\rho=\mathbb{E} n_{i}=z \tilde{f}^{\prime}(z),
$$

where, as in the introduction, we introduced the free energy per site $\mathfrak{f}(z)=\log z(z)$. The steady state current corresponding to the activity $z$ can be calculated as the average number of particles leaving a site

$$
j_{s t}(\rho(z))=\mathbb{E} \sum_{m=0}^{n_{i}} m \varphi\left(m \mid n_{i}\right)=z \frac{d}{d z} \log \mathfrak{v}(z),
$$

where $\mathfrak{b}(z)=\sum_{n \geq 0} z^{n} v(n)$ is the generating function of parameter $v(n)$. Substituting, for the case $q=v=0$ and $\mu=p$,

$$
\Varangle(z)=\frac{1}{1-z}, \quad \mathfrak{v}(z)=\frac{1}{1-p z},
$$

we obtain the following quantities

$$
\begin{gathered}
\rho=\frac{z}{1-z}, \\
j_{\mathrm{st}}=\frac{p z}{1-p z} \\
\theta=\frac{(1-p) p z^{2}}{(p z-1)^{2}}, \\
\chi=\frac{p(z-1)^{2}}{(p z-1)^{2}}
\end{gathered}
$$

as a function of the activity $z$ taking values in the range $z \in[0,1)$, when the density varies from zero to infinity. To find the explicit dependence of any of the quantities on any other, it is necessary to eliminate parameter $z$ between two equations. In the present case, it is a solvable a problem reducible to solving quadratic equations. However, in general, for example, for nonzero parameters $q$ and $v$, the parametric form of the functions in terms of mutual dependence on the parameter $z$ is the only way to write them in a relatively simple way. In what follows, we will often use some of these quantities as well as their derivatives, considering them as functions of some others. In that case we will write for example $\chi(\theta)$ or $\theta(\chi), \chi^{\prime}(\theta)$, etc., so that it is clear from the notation which quantity plays the role of a function, and which is an argument.

Let us now turn to the fluctuating part of the discussed random variables, corresponding to CLT. To compare the exact results with the scaling hypothesis (69) based on the analysis of the KPZ equation, it is necessary to express the dimensional constants through the parameters of the interface associated with the particle system. The hypothesis that makes this possible is based on a simple observation [81, 82] that one can construct only two dimensional invariants that do not change under scale transformations from the three parameters of the KPZ equation. One of them is $A$, defined in (67), and the other is the nonlinearity coefficient $\lambda$ from the original Eq. (66). The entire dimensional dependence of the average values characterizing statistics of fluctuations of the growing interface described by the KPZ equation is expressed in terms of combinations of these two parameters, which, in particular is consistent with the scaling hypothesis (69). It is reasonable to expect this fact to be true for all growing interfaces in the whole universality class. However, to use it, one should give a universal meaning to the invariants, not limited by the applicability of Eq. (66). Indeed, the parameter $A$ can be understood as the amplitude of the variance (68). To interpret the nonlinearity coefficient, we can consider the solution of the KPZ equation, with the initial condition obtained by adding a constant slope to the stationary solution $\kappa, h_{0}(x)=B_{x}+\kappa x$. Such an interface will move with constant speed 
$V_{\text {int }}(\kappa)=\mathbb{E}\left(d h_{t}(x) / d t\right)$ with the following slope depen-

dence $\lambda=v_{\text {int }}^{\prime \prime}(\kappa)$. In terms of the particle system, the mean velocity of the associated interface corresponds to the current, $v_{\text {int }}=j_{\text {st }}(\rho)$, and the slope corresponds to the particle density, $\rho=\kappa$, so that for the nonlinearity coefficient will have

$$
\lambda=\frac{d^{2} j_{\mathrm{st}}(\rho)}{d \rho^{2}} .
$$

For our ZRP the quantities $A$ and $\lambda$ can also be calculated in terms of the characteristics of the stationary state of the particle system and written via the activity $z$. In particular, the difference in the interface heights at two points under the variance is equal to the sum of occupation numbers between the two sites $h_{t}(x)-h_{t}(y)=\sum_{i=x+1}^{y} n_{i}$. As in the stationary state the occupation numbers of different sites are independent, the variance of the sum is given by the sum of the variances at each site, i.e.

$$
A=\mathbb{V} n_{i}=\left(z \frac{d}{z}\right)^{2} \mathfrak{f}(z)=z \frac{d \rho}{d z} .
$$

Substituting (76)-(79) in the last two relations we obtain

$$
A=\frac{z}{(1-z)^{2}}
$$

and

$$
\lambda=-\frac{2(1-p) p(1-z)^{3}}{(1-p z)^{3}} .
$$

Let us fix the position $x=\chi t$ on the macroscopic scale and look at the family of random variables that are obtained when considering interface heights at various points in the vicinity of this position at distances of the order of the correlation length (70). Summarizing all of the above, our scaling hypothesis for the interface height takes the form of the sum of two parts, deterministic of order of $t$ and random of order of $t^{1 / 3}$.

$$
\begin{gathered}
h_{t}\left(\chi t+u \sigma_{c}(\chi) t^{2 / 3}\right)=\underset{t}{\theta}(\chi) t+\sigma_{f}(\chi) t^{1 / 3} \mathscr{X}(u)+o\left(t^{1 / 3}\right) \\
t \rightarrow \infty .
\end{gathered}
$$

Here, the fluctuation and correlation dimensional parameters have the form,

$$
\begin{gathered}
\sigma_{f}=\left(\frac{|\lambda| A^{2}}{2}\right)^{1 / 3}=\frac{((1-p) p)^{1 / 3} z^{2 / 3}}{(1-p z)(1-z)^{1 / 3}}, \\
\sigma_{c}=\frac{1}{A}\left(\frac{|\lambda| A^{2}}{2}\right)^{2 / 3}=\frac{((1-p) p)^{2 / 3} z^{1 / 3}(1-z)^{4 / 3}}{(1-p z)^{2}} .
\end{gathered}
$$

In particular, for the one-point distribution, this is equivalent to the statement of the existence of the limiting distribution

$$
F_{\mathscr{Q}}(a)=\lim _{t \rightarrow \infty} \mathbb{P}\left(\frac{h_{t}(\chi t)-t \theta(\chi)}{\sigma_{f}(\chi) t^{1 / 3}}<a\right),
$$

and of the multipoint distributions

$$
\begin{gathered}
F_{X}^{u_{1}, \ldots, u_{n}}\left(a_{1}, \ldots, a_{n}\right) \\
=\lim _{t \rightarrow \infty} \mathbb{P}\left(\bigcap_{i=1}^{n}\left[\frac{h_{t}\left(\chi_{i} t\right)-t \theta\left(\chi_{i}\right)}{\sigma_{f}(\chi) t^{1 / 3}}<a_{i}\right]\right),
\end{gathered}
$$

where we used the notation $\chi_{i}=\chi+t^{-1 / 3} \sigma_{c} u_{i}$ for different points separated by distances of the order of the correlation length. Note that the dimensional parameters $\sigma_{f}(\chi)$ and $\sigma_{c}(\chi)$ depend on the point $\chi=x / t$ on the macroscopic scale. Nevertheless, as we will see from the exact solution, lhs of Eqs. (80), (81) do not depend on this point, indicating the universality of the obtained distributions.

In the previous section, we made a statement about the type of joint distributions of points of exits of particles from the boundaries, the simplest example of which is the distribution of coordinates of several particles at a fixed time. From the distribution of the interface height one can proceed to distributions of various observables characterizing the associated system of particles. For example, note that that event $x_{n}(t)<a$ is equivalent to event $h_{t}(a)<n$ or $\mathbb{P}\left(x_{n}(t)<a\right)=\mathbb{P}\left(h_{t}(a)<n\right)$. Therefore, for $(80)$ we will have

$$
\begin{gathered}
\mathbb{P}\left(h_{t}(\chi t)<t \theta(\chi)+a \sigma_{f}(\chi) t^{1 / 3}\right) \\
=\mathbb{P}\left(x_{t \theta(\chi)+a \sigma_{f}(\chi) t^{1 / 3}}<\chi t\right) .
\end{gathered}
$$

Basing on the law of large numbers (75) for $x_{n}(t)$ we suggest that

$$
x_{\theta t}(t)=t \chi(\theta)+\xi_{\theta}
$$

where $\xi_{\theta}=O\left(t^{1 / 3}\right)$ is a random variable. Then

$$
\begin{gathered}
\mathbb{P}\left(x_{t \theta(\chi)+a \sigma_{f}(\chi) t^{1 / 3}}<\chi t\right) \\
=\mathbb{P}\left(t \chi\left(\theta(\chi)+a \sigma_{f}(\chi) t^{-2 / 3}\right)+\xi_{\theta(\chi)+a \sigma_{f}(\chi) t^{-2 / 3}}<\chi t\right) \\
\simeq \mathbb{P}\left(\xi_{\theta(\chi)}<a \rho^{-1} \sigma_{f}(\theta) t^{1 / 3}\right),
\end{gathered}
$$

where we used the fact that $d \chi / d \theta=-1 / \rho$, and neglected the shift in the $\xi_{\theta}$ argument. As a result, from (80) we have

$$
F_{\mathscr{Q}}(a)=\lim _{t \rightarrow \infty} \mathbb{P}\left(\frac{x_{\theta t}(t)-t \chi(\theta)}{\rho^{-1} \sigma_{f}(\chi) t^{1 / 3}}<a\right) .
$$


In similar arguments for multipoint distributions, we should take into account the shifts of reference points of the order of the dynamic correlation length $\xi(t)=\sigma_{c} t^{3 / 2}$ from the initial macroscopic position

$$
\begin{gathered}
F_{X(u)}^{u_{1}, \ldots, u_{n}}\left(a_{1}, \ldots, a_{n}\right) \\
=\lim _{t \rightarrow \infty} \mathbb{P}\left(\bigcap_{i=1}^{n}\left[\frac{x_{t \theta_{i}}(t)-t \chi\left(\theta_{i}\right)}{\rho^{-1} \sigma_{f} t^{1 / 3}}<a_{i}\right]\right),
\end{gathered}
$$

where $\theta_{i}=\theta-\rho u_{i} \sigma_{c} t^{-1 / 3}$. Note that a factor appeared in the shift from the macroscopic position $\theta$ is $(-\rho)=d \theta / d \chi$.

To explain how, knowing the joint distributions of heights at a fixed time, to obtain distributions at different times, we will use the slow decorrelation phenomenon discovered in the works [83, 84]. As already noted, the characteristic correlation length, at which decorrelation of random variables describing the growing interface of the KPZ class occurs, has the order of growth $\xi(t) \asymp t^{2 / 3}$. Fluctuations of heights at points separated by distances much smaller than $\xi(t)$ are fully correlated, i.e. they can be considered identical random variables. Fluctuations at points at a distances of the order of $\xi(t)$ are already different, nontrivially correlated random variables. At distances much larger than $\xi(t)$, the height fluctuations can be considered independent. This also holds true if we consider different points at different times, with the time difference of the same order provided that the points under consideration are not on the same characteristic of the Euler equation (71).

$$
x=j^{\prime}(\rho) t
$$

The slow decorrelation is observed along the characteristic, i.e. fluctuations heights $\delta h_{t}(x)=\left(h_{t}(x)-t \theta(x / t)\right)$ remain completely correlated up to the scale of order of $O(t)$. Directions along characteristics are called time-like, and all the others are called space-like Using slow decorrelation, one can reduce the joint distributions of height fluctuations at different points of the space-time plane, separated by space-like intervals, to joint distributions at a fixed time. Indeed, the identity statement is written as

$$
\begin{aligned}
& h_{t+\delta t}(\chi t)-(t+\delta t) \theta\left(\frac{\chi t}{t+\delta t}\right) \\
= & h_{t}\left(\chi t\left(1+\frac{\delta t}{t}\right)^{-1}\right)-t \theta\left(\frac{\chi t}{t+\delta t}\right) .
\end{aligned}
$$

In particular, taking $\delta t=s \sigma_{c} \chi^{-1} t^{2 / 3}$, where $s$ is a dimensionless parameter specifying the time deviations on the characteristic scale, we obtain

$$
\begin{gathered}
h_{t+s \sigma_{c} \chi^{-1} t^{2 / 3}}\left(t \chi+u \sigma_{c} t^{2 / 3}\right)=h_{t}\left(\frac{\chi t+u \sigma_{c} t^{2 / 3}}{1+s \sigma_{c} \chi^{-1} t^{-1 / 3}}\right) \\
+s \sigma_{c} \chi^{-1} t^{2 / 3} \theta\left(\frac{\chi t+u \sigma_{c} t^{2 / 3}}{t+s \sigma_{c} \chi^{-1} t^{2 / 3}}\right) \simeq\left(t+s \sigma_{c} \chi^{-1} t^{2 / 3}\right) \\
\times \theta\left(\frac{\chi+u \sigma_{c} t^{-1 / 3}}{1+s \sigma_{c} \chi^{-1} t^{-1 / 3}}\right)+\delta h_{t}\left(\chi t+(u s) \sigma_{c} t^{2 / 3}\right) .
\end{gathered}
$$

Let us fix $n$ space-time positions $\left(b_{1}, \ldots, b_{n}\right)=\left(\left(x_{1}, t_{1}\right), \ldots,\left(x_{n}, t_{n}\right)\right)$, where

$$
t_{i}=t+s \sigma_{c} \chi^{-1} t^{2 / 3}, x_{i}=t \chi_{i}=t \chi+u_{i} \sigma_{c} t^{2 / 3} .
$$

Then

$$
\begin{aligned}
\lim _{t \rightarrow \infty} \mathbb{P}\left(\bigcap_{i=1}^{n}\left[\frac{h_{t_{i}}\left(x_{i}\right)-t_{i} \theta\left(\frac{x_{i}}{t_{i}}\right)}{\sigma_{f}(\chi) t^{1 / 3}}<a_{i}\right]\right) \\
=F_{\mathscr{L}(u)}^{u_{1}-S_{1}, \ldots, u_{n}-s_{n}}\left(a_{1}, \ldots, a_{n}\right) .
\end{aligned}
$$

An equivalent statement of joint distribution function of particle positions at different times will have the form

$$
\begin{gathered}
\lim _{t \rightarrow \infty} P\left(\bigcap_{i=1}^{n}\left[\frac{x_{t_{i} \theta\left(x_{i} / t_{i}\right)}\left(t_{i}\right)-x_{i}}{\rho^{-1} \sigma_{f}(\chi) t^{1 / 3}}<a_{i}\right]\right) \\
=F_{\mathscr{Q}(u)}^{u_{1}-s_{1}, \ldots, u_{n}-s_{n}}\left(a_{1}, \ldots, a_{n}\right) .
\end{gathered}
$$

We see that deviations from the macroscopic scale in time and space appeared together as a difference.

Note that the phenomenon of slow decorrelation was discovered using purely probabilistic arguments. Below, we demonstrate formulas for the scaling limit of the distributions of points of particle exits from the boundaries. The result will be an illustration of the phenomenon of slow decorrelation obtained directly from the exact solutions.

The probability distribution of the interface height $h_{t}(x)$ can be thought of as the distribution of the time the particle number $h$ takes to go beyond the coordinate $x$. Similarly, the joint distribution of $\left(h_{t_{1}}(x), \ldots, h_{t_{n}}(x)\right)$ can be thought of as the corresponding multiparticle analogue. On the other hand, as we discussed above, the distribution of quantities $\left(h_{t}\left(x_{1}\right), \ldots, h_{t}\left(x_{n}\right)\right)$ is related to the distribution of coordinates reached by the particles by the time $t$. Recalling the results of the previous section, we see that both these examples give special cases of distribution of particle exit positions from a fixed boundary, where the boundaries correspond to straight lines $x=$ const and $t=$ const respectively. Using the above arguments, let us try to formulate a statement about the limiting distribution of the positions of exits from an 
arbitrary boundary $\mathscr{B}$, naturally parameterized by coordinate $\tau=t-x$. More precisely, we will consider the sequence of the boundaries $\left\{\mathscr{B}^{(L)}\right\}_{L \in N}$, which on a large scale $\tau=[L \psi], L \rightarrow \infty$, are approximated by some smooth curve $\mathscr{C}$,

$$
b(\tau)=(x(\tau), \quad t(\tau)) \simeq L\left(\frac{\zeta(\psi)-\psi}{2}, \frac{\zeta(\psi)+\psi}{2}\right) .
$$

Here $\zeta(\psi)$ is a smooth function that defines the limiting boundary shape, and the approximate equality symbol means that the pointwise difference of the right and left sides is of order of $o\left(t^{1 / 3}\right)$, i.e. small compared to the fluctuation scale. From restrictions on geometry of boundaries the function $\zeta(\psi)$ is constrained by

$$
\frac{d x}{d \tau}=\frac{\zeta^{\prime}(\psi)-1}{2} \leq 0, \frac{d t}{d \tau}=\frac{\zeta^{\prime}(\psi)+1}{2} \geq 0,
$$

i.e.

$$
\left|\zeta^{\prime}(\psi)\right| \leq 1
$$

Let $T_{n}^{\mathscr{P}}$ denotes a random variable equal to the number $\tau$ of the site via which the particle with number $n$ leaves the boundary $\mathscr{B}$, and

$$
\psi(\eta)=\lim _{L \rightarrow \infty} \frac{T_{[\eta L]}^{\mathscr{P}}}{L}
$$

is its rescaled limiting value in the sense of the LLN. It can be defined as a solution to the equation

$$
(\zeta(\psi)-\psi)=\chi\left(\frac{2 \eta}{\zeta(\psi)+\psi}\right)(\zeta(\psi)+\psi),
$$

obtained by rewriting the LLN (75) in terms of $\psi$.

Next, applying an argument similar to the one used above for relating distributions of $h_{t}(x)$ and $x_{n}(t)$, $\mathbb{P}\left(T_{n}^{\mathscr{P}}<a\right)=\mathbb{P}\left(h_{t(a)}(x(a))<n\right)$, we obtain

$$
F_{\mathscr{L}}(a)=\lim _{t \rightarrow \infty} \mathbb{P}\left(\frac{T_{[\eta L]}^{\mathscr{B}}-L \psi(\eta)}{\psi^{\prime}(\eta) \sigma_{f}(\chi) t^{1 / 3}}<a\right) .
$$

Here the dimensional factor $\psi^{\prime}(\eta)$ follows from differentiation of the relation (83) and is equal to

$$
\psi^{\prime}(\eta)=\frac{2 \rho^{-1}}{\left(\zeta^{\prime}(\psi)+1\right)\left(\chi+\theta \rho^{-1}\right)+\left(1-\zeta^{\prime}(\psi)\right)},
$$

where the values $\theta, \rho=\rho(\theta)$ and $\chi=\chi(\theta)$ are calculated for the value of the argument

$$
\theta=\frac{n}{t}=\frac{2 \eta}{\zeta(\psi)+\psi}
$$

To write a direct generalization of the formula (84) for multipoint distributions that can then be interpreted in terms of random processes on space-like paths, consider instead of one curve, which was deter- mined by the function $\zeta(\psi)$, a one-parameter family of curves $\left\{\mathscr{C}_{\omega}\right\}_{\omega \in R}$, defined by the functions $\zeta(\psi, \omega)$. With a fixed value of parameter $\omega$ the function $\zeta(\psi, \omega)$ as before gives a smooth curve in the $x-t$ plane. When the parameter $\omega$ runs through all real values, the curve deforms smoothly, shifting right and down as $\omega$ increases, so that $\mathscr{C}_{\omega_{1}} \preceq \mathscr{C}_{\omega_{2}}$ for any two values $\omega_{1}<\omega_{2}$. The macroscopic value $\psi(\eta, \omega)$ of the number of the exit position of the particle with number $n=[\eta L]$ from the boundary $\mathscr{B}_{\omega}^{(L)} \simeq L C_{\omega}$ will still be given by the solution of Eq. (83), although now, in addition to depending on the particle number, it will be a function of the boundary parameter $\omega$.

Now consider the smooth path $\Gamma=\{\eta(r), \omega(r)\}_{r \in \mathbb{R}}$ in the plane $\eta-\omega$, such that $\omega^{\prime}(r) \geq 0, \eta^{\prime}(r)>0$. Thus, to each curve $\mathscr{C}_{\omega(r)}$ we associate a particle with number $[L \eta(r)]$, in which exit from the boundary $\mathscr{B}_{\omega(r)}^{(L)} \simeq L C_{\omega(r)}$ we will be interested. Take $n$ points $r_{1}, \ldots, r_{n}$ on path $\Gamma$ so that the corresponding points in the $x-t$ plane are separated from each other by distances of the order of $O\left(L^{2 / 3}\right)$,

$$
r_{i}=r_{0}+u_{i}(d \psi / d r)^{-1} \sigma_{c} L^{-1 / 3}
$$

Here, guided by the same principle that was used above, to switch between distributions of different random variables, we have included in the scale of the parameter $r$ the dimensional factor

$$
\frac{d \psi}{d r}=\left.\frac{\zeta^{(0,1)}\left(1-\chi-\theta \rho^{-1}\right) \omega^{\prime}+2 \rho^{-1} \eta^{\prime}}{\left(\zeta^{(1,0)}+1\right)\left(\chi+\theta \rho^{-1}\right)+\left(1-\zeta^{1,0}\right)}\right|_{r=r_{0}},
$$

which is again obtained by differentiating the formula (83), now with respect to $r$, also taking into account the dependence of $\zeta(\psi, \omega)$ on the second argument. After that the values of parameters indexing particles and boundaries are taken in the form

$$
\omega_{i}=\omega\left(r_{i}\right), \quad \eta_{i}=\eta\left(r_{i}\right),
$$

see Fig. 6. Then the multipoint analogue of the formula (84) will look as follows

$$
\begin{gathered}
\lim _{L \rightarrow \infty} \mathbb{P}\left(\bigcap_{i=1}^{n}\left[\frac{T_{\left[\eta_{i} L\right]}^{L C_{\omega_{i}}}-L \psi\left(\eta_{i}, \omega_{i}\right)}{(\partial \psi / \partial \eta) \sigma_{f}(\chi) t^{1 / 3}}<a_{i}\right]\right) \\
=F_{\mathscr{Q}(u)}^{u_{1}, \ldots, u_{n}}\left(a_{1}, \ldots, a_{n}\right) .
\end{gathered}
$$

Note that the coefficient of the parameter $u$ in the formula (83) diverges when the numerator in the formula (86) vanishes. It corresponds to situations, when the path $\Gamma$ is on the characteristic of the Euler equation, i.e. time-like, and the absence of dependence of the resulting random process on coordinates on the path on the considered scale reflects the slow decorrelation phenomena. 


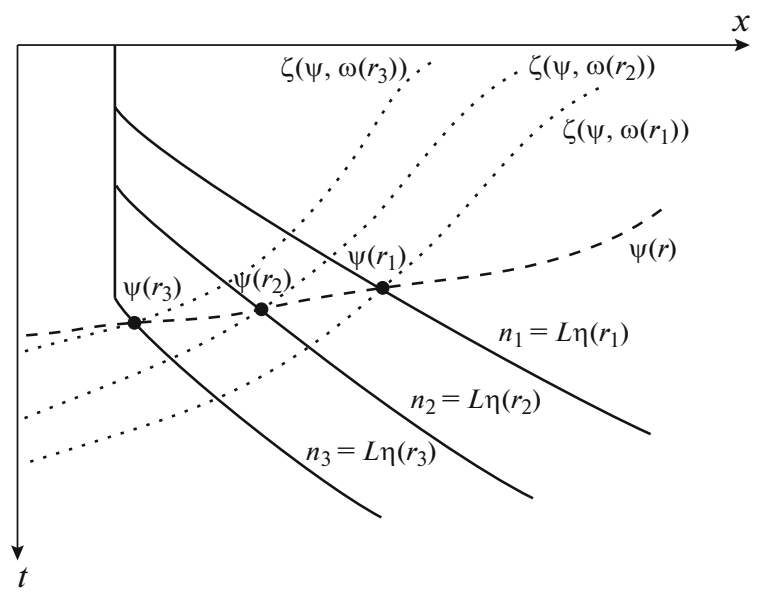

Fig. 7. Distribution of exit positions from the boundaries on space-like paths in the $x-t$ plane. Solid lines show the macroscopic deterministic trajectories of particles numbered $n_{i}=\left[\operatorname{L\eta }\left(r_{i}\right)\right], i=1,2,3$. The dotted lines show the smooth limits of the three boundaries, $\mathscr{B}_{\omega\left(r_{i}\right)}^{(L)} \simeq L C_{\omega\left(r_{i}\right)}$, whose positions $\quad(x, t) \simeq L\left(\frac{\zeta\left(\psi, \omega\left(r_{i}\right)\right)-\psi}{2}, \frac{\zeta\left(\psi, \omega\left(r_{i}\right)\right)+\psi}{2}\right)$ are defined by the function $\zeta(\psi, \omega)$ with fixed values of the second argument. The points of their intersections (black dots) define the coordinates $\psi\left(r_{i}\right)$ as solutions to Eq. (83). The dashed line shows a space-like path: by specifying the functions $g a(r)$ and $\eta(r)$, we respectively define $\psi(r)$ for the family of boundaries. Fluctuations of a point in a neighborhood of $\psi(r)$ converge to one universal limit for almost all space-time paths.

Above, we have demonstrated a collection of scaling hypotheses about the convergence of different multipoint distributions to one universal limit. To describe this limit, we need to use the results of the exact solution described in the previous section. There, we represented the exact expression for the probabilities of exit from the boundaries in the form of the Fredholm determinant. To investigate to what this expression converges in the scaling limit (87) one should perform an asymptotic analysis of the Fredholm determinant, the technical part of which consists in the analysis of integral representation of the correlation kernel by the saddle method, and the proof of the uniform convergence of the series representing the Fredholm determinant. Here we omit the details of the asymptotic analysis. An interested the reader can find them in the works [40, 41, 76-78]. We will only formulate the answer by presenting explicit formulas for the limiting distributions.

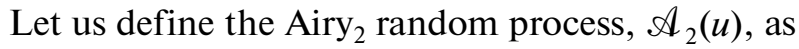
stationary random process with the following finitedimensional distributions.

$$
\begin{aligned}
& \operatorname{Prob}\left(\mathscr{A}_{2}\left(u_{1}\right)<s_{1}, \ldots, \mathscr{A}_{2}\left(u_{m}\right)<s_{m}\right) \\
& \left.=\operatorname{det}\left(1-\eta_{s} K_{\text {Airy }} \eta_{s}\right)\right)_{L^{2}\left(\left\{\eta_{1}, \ldots, n_{m}\right\} \times \mathbb{R}\right)}
\end{aligned}
$$

where $K_{\text {Airy }}$ is an integral operator with kernel

$$
=\left\{\begin{array}{c}
K_{\text {Airy }}\left(\xi_{1}, \zeta_{1}, \xi_{2}, \zeta_{2}\right) \\
\int_{0}^{\infty} d \lambda e^{\lambda\left(\xi_{2}-\xi_{1}\right)} \operatorname{Ai}\left(\lambda+\zeta_{1}\right) \operatorname{Ai}\left(\lambda+\zeta_{2}\right), \quad \xi_{2} \leq \xi_{1} ; \\
-\int_{-\infty}^{0} d \lambda e^{\lambda\left(\xi_{2}-\xi_{1}\right)} \operatorname{Ai}\left(\lambda+\zeta_{1}\right) \operatorname{Ai}\left(\lambda+\zeta_{2}\right), \quad \xi_{2}>\xi_{1} .
\end{array}\right.
$$

The one-point distribution of this process is the famous Tracy-Widom distribution [6], which is given by a similar Fredholm determinant on $L^{2}(\mathbb{R})$ with the Airy kernel

$$
K_{\text {Airy }}(x, y)=\frac{A i(x) A i^{\prime}(y)-A i(y) A i^{\prime}(x)}{x-y} .
$$

The Tracy-Widom distribution was first discovered in the theory of random matrices [8], where it describes the distribution of a properly rescaled largest (and smallest) eigenvalue of a random Hermitian matrix from the Gaussian unitary ensemble. The matrix elements of matrices from this ensemble are independent normally distributed random variables. If we think of the matrix elements as independent Brownian motions with the same one-time distributions [85], then the dependence of the largest eigenvalue on time will be described by the Airy ensemble. $_{2}$ ens

Using the notations introduced after the formula (87), we formulate the following statement Theorem 2.2 [41].

Let

$$
\begin{gathered}
r=r_{0}+u(d \psi / d r)^{-1} \sigma_{c} L^{-1 / 3}, \\
\eta_{u}=\eta(r), \quad \omega_{u}=\omega(r) .
\end{gathered}
$$

The following limit holds in the sense of finitedimensional distributions

$$
\lim _{L \rightarrow \infty} \frac{T_{\left[L \eta_{u}\right]}^{L \mathscr{C}_{\omega_{u}}}-L \psi\left(\eta_{u}, \omega_{u}\right)}{\psi^{\prime}(\eta) \sigma_{f}(\chi) t^{1 / 3}}=\mathscr{A}_{2}(u) .
$$

From this it can be seen, that random variables describing the positions in which the trajectories of interacting particles intersect lines from different oneparameter families converge to the same universal random process after the propoer choice of units. For the family of curves defined by the function $\zeta(\psi, \omega)$ and for almost any choice of a path $(\eta(r), \omega(r))$ the scaling model-dependent parameters are easily reconstructed from the formulas given, except for the case of timelike paths, when the correlation length is of different scale. The simplest examples of the $\zeta(\psi, \omega)$ function reproduce special cases of statistics that were studied earlier. For example, for the case of one purely spatial boundary allowing a characterization of the joint distribution of particle positions at a fixed time $t$, we set $\zeta(\psi, \omega)=2 t / L-\psi$. To investigate the current correlation functions [40], i.e. distribution of the times of 
reaching a given coordinate $x$ by different particles, we must consider $\zeta(\psi, \omega)=\psi-2 x / L$. To consider the positions of particles along space-like paths [86, 87], one can include a non-trivial dependence on $\omega$ in $\zeta(\psi, \omega), \zeta(\psi, \omega)=\omega-\psi$. Note that the results of $[86,87]$ assumed an additional temporal ordering of points on the considered space-like paths, while in our case the use of generalized Green functions allowed us to get rid of this restriction. Another case, which can be obtained, as a special case of our formulas, is the case of the joint multipoint distribution of the positions of the tagged particle, which was studied in [88]. It corresponds to a special path selection, $\eta(r)=$ const .

Thus, we have illustrated the convergence of the wide class of statistics, describing the probabilities for the interacting particles to go beyond arbitrary spacetime boundaries, to the limiting Airy 2 process, which is supposedly universal in the KPZ class. There are also other limiting processes corresponding to a different global shape of initial conditions. Their description is beyond the scope of our review.

\section{REFERENCES}

1. A. Ya. Khinchin, "Statistical mechanics as a problem of probability theory," Usp. Mat. Nauk 5 (3), 3-46 (1950).

2. R. L. Dobrushin an B. Tirozzi, "The central limit theorem and the problem of equivalence of ensembles," Commun. Math. Phys. 54, 173-192 (1977).

3. M. Campanino, G. Del Grosso, and B. Tirozzi, "Local limit theorem for Gibbs random fields of particles and unbounded spins," J. Math. Phys. 20, 1752-1758 (1979).

4. M. Kardar, G. Parisi, and Y. C. Zhang, "Dynamic scaling of growing interfaces," Phys. Rev. Lett. 56, 889 (1986)

5. T. Halpin-Healy and Y. C. Zhang, "Kinetic roughening phenomena, stochastic growth, directed polymers and all that. Aspects of multidisciplinary statistical mechanics,” Phys. Rep. 254 (4-6), 215-414 (1995).

6. C. A. Tracy and H. Widom, "Level-spacing distributions and the Airy kernel," Commun. Math. Phys. 159, 151-174 (1994).

7. M. Prähofer and H. Spohn, "Scale invariance of the PNG droplet and the Airy process," J. Stat. Phys. 108 (5-6), 1071-1106 (2002).

8. M. L. Mehta, Random Matrices (Elsevier, Amsterdam, 2004).

9. J. Baik, P. Deift, and K. Johansson, "On the distribution of the length of the longest increasing subsequence of random permutations,” J. Am. Math. Soc. 12, 11191178 (1999).

10. A. M. Vershik and S. V. Kerov, "Asymptotics of the Plancherel measure of the symmetric group and the limiting form of Young tableaux," Dokl. Akad. Nauk 233, 1024-1027 (1977).
11. W. Jockusch, J. Propp, and P. Shor, "Random domino tilings and the Arctic Circle theorem," arXiv: math/9801068 (1998).

12. K. Johansson, "The Arctic Circle boundary and the Airy process," Ann. Probab. 33 (1), 1-30 (2005).

13. P. L. Ferrari and H. Spohn, "Constrained Brownian motion: Fluctuations away from circular and parabolic barriers," Ann. Probab. 33, 1302-1325 (2005).

14. K. Johansson, "Shape fluctuations and random matrices," Commun. Math. Phys. 209, 437-476 (2000).

15. M. Kardar, "Directed paths in random media," arXiv: cond-mat/9411022 (1994).

16. E. H. Lieb and W. Liniger, "Exact analysis of an interacting Bose gas. I. The general solution and the ground state," Phys. Rev. 130, 1605 (1963).

17. G. Amir, I. Corwin, and J. Quastel, "Probability distribution of the free energy of the continuum directed random polymer in 1+1 dimensions," Commun. Pure Appl. Math. 64, 466-537 (2011).

18. P. Calabrese, P. Le Doussal, and A. Rosso, "Free-energy distribution of the directed polymer at high temperature," Europhys. Lett. 90, 20002 (2010).

19. V. Dotsenko, "Two-temperature statistics of free energies in $(1+1)$ directed polymers," Europhys. Lett. 116, 40004 (2017).

20. T. Sasamoto and H. Spohn, "One-dimensional Kardar-Parisi-Zhang equation: An exact solution and its universality,” Phys. Rev. Lett. 104, 230602 (2010).

21. V. E. Korepin, N. M. Bogoliubov, and A. G. Izergin, Quantum Inverse Scattering Method and Correlation Functions (Cambridge Univ. Press, 1997).

22. L. H. Gwa and H. Spohn, "Six-vertex model, roughened surfaces, and an asymmetric spin Hamiltonian," Phys. Rev. Lett. 68, 725 (1992).

23. H. Bethe, "On the theory of metals, I. Eigenvalues and eigenfunctions of a linear chain of atoms," in Selected Works of Hans A. Bethe: With Commentary (World Scientific, 1997), pp. 155-183.

24. A. A. Litvin and V. B. Priezzhev, "The Bethe ansatz for the six-vertex model with rotated boundary conditions," J. Stat. Phys. 60, 307-321 (1990).

25. S. Karlin and J. McGregor, "Coincidence probabilities,” Pac. J. Math. 9, 1141-1164 (1959).

26. B. Lindström, "On the vector representations of induced matroids," Bull. London Math. Soc. 5, 85-90 (1973).

27. I. M. Gessel and X. Viennot, "Determinants, paths, and plane partitions," Preprint 1989, http://people.brandeis.edu/ gessel/homepage/papers/pp.pdf.

28. R. Brak, J. Essam, J. Osborn, A. L. Owczarek, and A. Rechnitzer, "Lattice paths and the constant term," J. Phys.: Conf. Ser. 42, 47 (2006).

29. R. Brak and W. Galleas, "Constant term solution for an arbitrary number of osculating lattice paths," Lett. Math. Phys. 103, 1261-1272 (2013).

30. B. Eynard and M. L. Mehta, "Matrices coupled in a chain: I. Eigenvalue correlations," J. Phys. A: Math. Gen. 31, 4449 (1998).

31. A. Borodin and E. M. Rains, "Eynard-Mehta theorem, Schur process, and their Pfaffian analogs," J. Stat. Phys. 121, 291-317 (2005). 
32. J. Krug, "Origins of scale invariance in growth processes,” Adv. Phys. 46, 139-282 (1997).

33. P. L. Ferrari, "From interacting particle systems to random matrices," J. Stat. Mech.: Theory Exp. No. 10, 10016 (2010).

34. A. Borodin and V. Gorin, "Lectures on integrable probability," Probab. Stat. Phys. St. Petersburg 91, 155-214 (2016).

35. J. Quastel, "Introduction to KPZ," Curr. Dev. Math., No. 1 (2011).

36. I. Corwin, "The Kardar-Parisi-Zhang equation and universality class," Random Matrices: Theory Appl. 1 (01), 1130001 (2012).

37. J. G. Brankov, V. B. Priezzhev, and R. V. Shelest, "Generalized determinant solution of the discrete-time totally asymmetric exclusion process and zero-range process," Phys. Rev. E 69, 066136 (2004).

38. V. B. Priezzhev, "Non-stationary probabilities for the asymmetric exclusion process on a ring," Pramana 64, 915-925 (2005).

39. A. M. Povolotsky and V. B. Priezzhev, "Determinant solution for the totally asymmetric exclusion process with parallel update," J. Stat. Mech.: Theory Exp., No. 07, P07002 (2006).

40. A. M. Povolotsky, V. B. Priezzhev, and G. M. Schütz, "Generalized Green functions and current correlations in the TASEP,” J. Stat. Phys. 142, 754-791 (2011).

41. S. S. Poghosyan, A. M. Povolotsky, and V. B. Priezzhev, "Universal exit probabilities in the TASEP," J. Stat. Mech.: Theory Exp., No. 08, P08013 (2012).

42. A. M. Povolotsky, "On the integrability of zero-range chipping models with factorized steady states," J. Phys. A: Math. Theor. 46, 465205 (2013).

43. M. E. Fisher, "Walks, walls, wetting, and melting," J. Stat. Phys. 34, 667-729 (1984).

44. H. Rosengren, "A non-commutative binomial formula,” J. Geom. Phys. 32, 349-363 (2000).

45. M. R. Evans, S. N. Majumdar, and R. K. Zia, "Factorized steady states in mass transport models," J. Phys. A: Math. Gen. 37, L275 (2004).

46. G. Barraquand and I. Corwin, "The $q$-Hahn asymmetric exclusion process," Ann. Appl. Probab. 26, 2304-2356 (2016).

47. A. Borodin, I. Corwin, L. Petrov, and T. Sasamoto, "Spectral theory for interacting particle systems solvable by coordinate Bethe ansatz," Commun. Math. Phys. 339, 1167-1245 (2015).

48. R. Frassek, "The non-compact XXZ spin chain as stochastic particle process," J. Phys. A: Math. Theor. 52, 335202 (2019).

49. G. Barraquand and I. Corwin, "Random-walk in Betadistributed random environment," Probab. Theory Relat. Fields 167, 1057-1116 (2017).

50. T. Sasamoto and M. Wadati, "One-dimensional asymmetric diffusion model without exclusion," Phys. Rev. E 58, 4181 (1998).

51. M. Alimohammadi, V. Karimipour, and M. Khorrami, "Exact solution of a one-parameter family of asymmetric exclusion processes,” Phys. Rev. E 57, 6370 (1998).

52. M. Alimohammadi, V. Karimipour, and M. Khorrami, "A two-parametric family of asymmetric exclusion processes and its exact solution,” J. Stat. Phys. 97, 373394 (1999).

53. G. M. Schütz, R. Ramaswamy, and M. Barma, "Pairwise balance and invariant measures for generalized exclusion processes," J. Phys. A: Math. Gen. 29, 837 (1996).

54. R. Frassek, C. Giardinà, and J. Kurchan, "Non-compact quantum spin chains as integrable stochastic particle processes," J. Stat. Phys. 180, 135-171 (2020).

55. S. E. Derkachov, "Baxter's Q-operator for the homogeneous XXX spin chain,” J. Phys. A: Math. Gen. 32, 5299 (1999).

56. N. M. Bogoliubov and R. K. Bullough, "A $q$-deformed completely integrable Bose gas model," J. Phys. A: Math. Gen. 25, 4057 (1992).

57. T. Sasamoto and M. Wadati, "Exact results for one-dimensional totally asymmetric diffusion models," J. Phys. A: Math. Gen. 31, 6057 (1998).

58. A. M. Povolotsky, "Bethe ansatz solution of zero-range process with nonuniform stationary state," Phys. Rev. E 69, 061109 (2004).

59. A. M. Povolotsky and J. F. Mendes, "Bethe ansatz solution of discrete time stochastic processes with fully parallel update," J. Stat. Phys. 123, 125-166 (2006).

60. A. Borodin and I. Corwin, "Macdonald processes," Probab. Theory Relat. Fields 158, 225-400 (2014).

61. A. Borodin, I. Corwin, and P. Ferrari, "Free energy fluctuations for directed polymers in random media in $1+1$ dimension," Commun. Pure Appl. Math. 67, 1129-1214 (2014).

62. V. B. Priezzhev, E. V. Ivashkevich, A. M. Povolotsky, and C. K. Hu, "Exact phase diagram for an asymmetric avalanche process," Phys. Rev. Lett. 87, 084301 (2001).

63. A. Borodin and I. Corwin, "Discrete time $q$-TASEPs," Int. Math. Res. Not., No. 2, 499-537 (2015).

64. A. Borodin and P. Ferrari, "Large time asymptotics of growth models on space-like paths I: PushASEP," Electron. J. Probab. 13, 1380-1418 (2008).

65. A. M. Povolotsky, V. B. Priezzhev, and C. K. Hu, "The asymmetric avalanche process," J. Stat. Phys. 111, 1149-1182 (2003).

66. J. F. Van Diejen, "Diagonalization of an integrable discretization of the repulsive delta Bose gas on the circle," Commun. Math. Phys. 267, 451-476 (2006).

67. I. Corwin and L. Petrov, "Stochastic higher spin vertex models on the line," Commun. Math. Phys. 343, 651700 (2016).

68. I. Corwin, K. Matveev, and L. Petrov, "The $q$-Hahn PushTASEP,” Int. Math. Res. Not. (2018), https://doi.org/10.1093/imrn/rnz106

69. G. M. Schütz, "Exact solution of the master equation for the asymmetric exclusion process," J. Stat. Phys. 88, 427-445 (1997).

70. A. Rákos and G. M. Schütz, "Current distribution and random matrix ensembles for an integrable asymmetric fragmentation process," J. Stat. Phys. 118, 511-530 (2005).

71. A. E. Derbyshev, S. S. Poghosyan, A. M. Povolotsky, and V. B. Priezzhev, "The totally asymmetric exclusion process with generalized update," J. Stat. Mech: Theory Exp., No. 05, P05014 (2012). 
72. C. A. Tracy and H. Widom, "Integral formulas for the asymmetric simple exclusion process," Commun. Math. Phys. 279, 815-844 (2008).

73. A. Borodin, I. Corwin, L. Petrov, and T. Sasamoto, "Spectral theory for the $q$-Boson particle system," Compos. Math. 151, 1-67 (2015).

74. R. P. Stanley, Enumerative Combinatorics, 2nd ed. (Cambridge Univ. Press, 2011), Vol. 1.

75. T. Sasamoto, "Spatial correlations of the 1D KPZ surface on a flat substrate," J. Phys. A: Math. Gen. 38, L549 (2005).

76. A. Borodin, P. L. Ferrari, M. Prähofer, and T. Sasamoto, "Fluctuation properties of the TASEP with periodic initial configuration," J. Stat. Phys. 129, 1055-1080 (2007).

77. A. Borodin, P. L. Ferrari, and M. Prähofer, "Fluctuations in the discrete TASEP with periodic initial configurations and the Airy1 process," Int. Math. Res. Pap. (2007), https://doi.org/10.1093/imrp/rpm002

78. A. Borodin, P. L. Ferrari, and T. Sasamoto, "Transition between Airy1 and Airy2 processes and TASEP fluctuations," Commun. Pure Appl. Math. 61, 1603-1629 (2008).

79. T. Nagao and T. Sasamoto, "Asymmetric simple exclusion process and modified random matrix ensembles," Nucl. Phys. B 699, 487-502 (2004).

80. F. Family and T. Vicsek, "Scaling of the active zone in the Eden process on percolation networks and the bal- listic deposition model," J. Phys. A: Math. Gen. 18, L75 (1985).

81. J. G. Amar and F. Family, "Critical cluster size: Island morphology and size distribution in submonolayer epitaxial growth,” Phys. Rev. Lett. 74, 2066 (1995).

82. J. Krug, P. Meakin, and T. Halpin-Healy, "Amplitude universality for driven interfaces and directed polymers in random media," Phys. Rev. A 45, 638 (1992).

83. P. L. Ferrari, "Slow decorrelations in Kardar-ParisiZhang growth,” J. Stat. Mech.: Theory Exp., No. 07, P07022 (2008).

84. I. Corwin, P. L. Ferrari, and S. Péché, "Universality of slow decorrelation in KPZ growth," Ann. l'IHP Probab. Stat. 48, 134-150 (2012).

85. F. J. Dyson, "A Brownian motion model for the eigenvalues of a random matrix," J. Math. Phys. 3, 11911198 (1962).

86. A. Borodin and P. Ferrari, "Large time asymptotics of growth models on space-like paths I: PushASEP," Electron. J. Probab. 13, 1380-1418 (2008).

87. A. Borodin, P. L. Ferrari, and T. Sasamoto, "Large time asymptotics of growth models on space-like paths II: PNG and parallel TASEP," Commun. Math. Phys. 283, 417-449 (2008).

88. T. Imamura and T. Sasamoto, "Dynamics of a tagged particle in the asymmetric exclusion process with the step initial condition," J. Stat. Phys. 128, 799-846 (2007). 